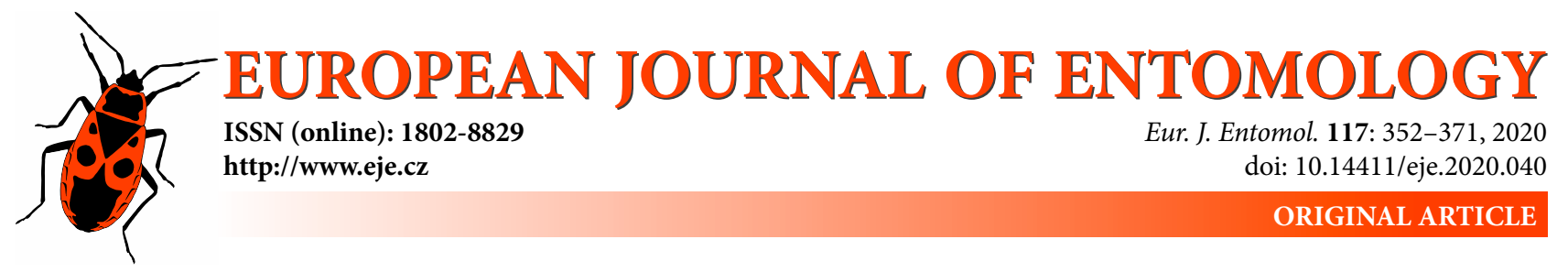

\title{
Composition and function of the microbiotas in the different parts of the midgut of Pyrrhocoris sibiricus (Hemiptera: Pyrrhocoridae) revealed using high-throughput sequencing of $16 S$ rRNA
}

\author{
RongRong $\mathrm{LI}^{1, *}$, MIN LI $^{1, *}$, JIANG YAN ${ }^{1}$ and HufANG ZHANG ${ }^{2, * *}$ \\ ${ }^{1}$ Taiyuan Normal University, Jinzhong, Shanxi 030619, China; e-mails: yanj@tynu.edu.cn, limin12nk@163.com, \\ 412656310@qq.com \\ ${ }^{2}$ Xinzhou Teachers University, Xinzhou, Shanxi 034000, China; e-mail: zh_hufang@sohu.com
}

Key words. Pyrrhocoridae, Pyrrhocoris sibiricus, digestion, gut microbiota, community structure, functional profiling, nextgeneration sequencing, 16S rRNA gene

\begin{abstract}
In pyrrhocorids, digestion of food occurs mainly in the midgut, which is divided into four parts (M1-M4), and takes between three and four days. Food is retained in M1 for about $5 \mathrm{~h}$ and passes quickly through M4. However, food is retained in M2 and M3 much longer, about 70 to $90 \mathrm{~h}$. The different stages in digestion may be influenced by different microbial populations in the different parts of the midgut. In the present study, the microbiota in the four parts of the midgut of Pyrrhocoris sibiricus were analysed in detail using high-throughput sequencing of the 16S rRNA V3-V4 region. The most abundant bacteria in M3 were Actinobacteria (Coriobacteriaceae) whereas it was Proteobacteria (gammaproteobacteria) in M1, M2 and M4. Actinobacteria was the second most abundant bacterial group in M2. According to the PCA analysis, M2 and M3 have the most similar bacterial communities. Burkholderia, which is closely related to the plant-associated beneficial and environmental (PBE) group, was also found in M1, M2 and M4. Predictive functional profiles of the metagenomes revealed that metabolism mostly occurred in M2 and M3. The PICRUSt results were consistent with the 16S rRNA metagenomic analysis and indicate that the bacteria in M2 and M3 play an important role in degrading complex dietary components.
\end{abstract}

\section{INTRODUCTION}

Symbiotic interactions between animals and beneficial microorganisms are very common in nature, including insects (Buchner, 1965; Smith, 1989; Moran et al., 2008). In many cases, these symbionts are housed in the gut of their hosts and play an essential nutritional role in maintaining the fitness of the host insect. Their role can involve detoxifying plant allelochemicals, promoting digestion and providing digestive enzymes or nutrients, especially when the insect is either a blood, sap or cellulose feeder (Buchner, 1965; Douglas, 1992; Akman et al., 2002; Genta et al., 2006; Sudakaran et al., 2012; Taylor et al., 2014; Onchuru et al., 2018).

The Pyrrhocoridae are terrestrial bugs of which there are $\sim 340$ species belonging to 33 genera worldwide (Schaefer \& Ahmad, 2000; Henry, 2009). Most of them prefer to feed on the seeds of Malvales plants, which are vitamin limited and avoided by other insects due to their phytochemical defences (Allen et al., 1967; Abou-Donia, 1976; Ahmad \& Schaefer, 1987; Kristenová et al., 2011). The midgut of Pyr- rhocoridae is differentiated into four distinct parts, namely M1-M4. The digestion of food in pyrrhocorids takes three or four days, with most of the time spent in M2 and M3 (Silva \& Terra, 1994). It is suggested that the final stages of digestion occur in the midgut as the salivary digestive enzymes in seed feeders only have a minor role (Saxena, 1963; Silva \& Terra, 1994). Previous studies on the gut microbiota of cotton stainers (Dysdercus fasciatus Signoret) and red firebugs (Pyrrhocoris apterus Linnaeus) indicate that a special microbiota, Actinobacteria, dominate in M3 and provide the host with B vitamins and protects it from parasites (Sudakaran et al., 2012; Salem et al., 2014; Onchuru et al., 2018). However, the role of microbiota in the digestion of food is still unclear.

Pyrrhocoris sibiricus Kuschakevich, a ground-dwelling seed-feeder, is widely distributed in Russian Far East, Central and East Mongolia, China, North Korea and Japan (Zhang, 1985; Matolin \& Štys, 1987). Both nymphs and adults of $P$. sibiricus feed on the seeds of leguminous and gramineous plants, which subsequently affects the health

\footnotetext{
* The first two authors contributed equally to the present study.

** Corresponding author; e-mail: zh_hufang@sohu.com
} 


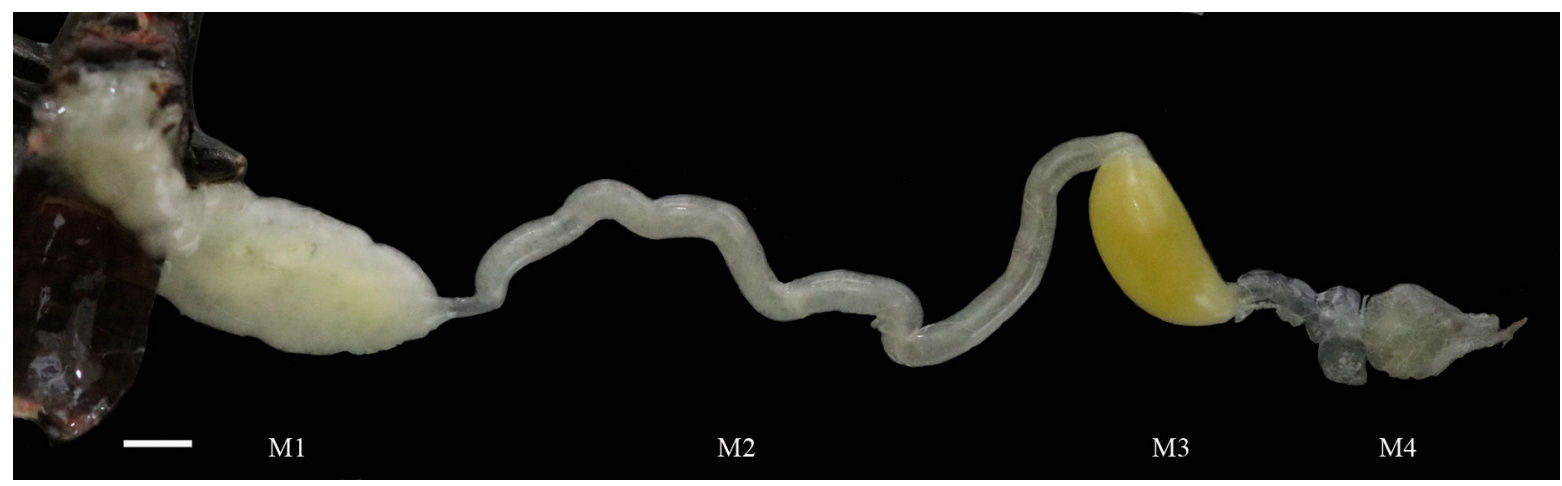

Fig. 1. Photograph of a dissected midgut of Pyrrhocoris sibiricus (M1-M4). Scale bar $=1 \mathrm{~mm}$.

of these plants (Zhang, 1985; Matolin \& Štys, 1987). In the present study, 16S rRNA metagenomic analysis was used to characterize the microbes that inhabit the different parts of the midgut of $P$. sibiricus. Furthermore, a PICRUSt (Phylogenetic Investigation of Communities by Reconstruction of Unobserved States) analysis was used to determine the functional potentials of the bacteria in the M1, M2, M3 and M4 using the 16S rRNA marker gene.

\section{MATERIALS AND METHODS}

\section{Sample collection}

Adult specimens of $P$. sibiricus feeding on the seeds of $A b u$ tilon theophrasti Medicus were collected between September and December 2018 in Jinzhong, Shanxi Province $\left(37.68^{\circ} \mathrm{N}\right.$, $112.75^{\circ} \mathrm{E}$ ). Prior to dissection, they were soaked in $70 \%$ ethyl alcohol for $3 \mathrm{~min}$ and then washed 3 times with sterile deionized water in order to remove exogenous contaminants. The guts were then dissected under sterile deionized water using sterilized tweezers and eye scissors under aseptic conditions. Each midgut was separated into four parts, M1, M2, M3 and M4 (Fig. 1), which were treated separately. Since there were three replicates for each part and each replicate consisted of a mixture of samples from 20 adults regardless of sex, a total of 60 adults were dissected. Five hundred milligrams of each part were collected and immediately frozen in liquid nitrogen for subsequent DNA extraction. The remains of the material of each of the parts were also frozen in liquid nitrogen and preserved at $-80^{\circ} \mathrm{C}$.

\section{DNA extraction and sequencing}

DNA was extracted using a Mag-Bind Soil DNA extraction kit (Omega, Norcross, GA, USA) according to the manufacturer's instructions with slight modifications to improve the efficiency of the DNA extraction from the gut samples. DNA concentration was measured using a NanoDrop ND-1000 spectrophotometer (Thermo Fisher Scientific, Waltham, MA, USA) and its quality confirmed using agarose gel electrophoresis. The V3-V4 hypervariable region of the $16 \mathrm{~S}$ rRNA gene was then amplified using PCR and the universal primers 338F (5'-ACTCCTACGGGAGGCAGCA-3') and 806R (5'-GGACTACHVGGGTWTCTAAT-3') (Mizrahi-Man et al., 2013). The reaction solution consisted of: $5 \mu$ of Q5 reaction buffer $(5 \times), 5 \mu$ of Q5 HighFidelity GC buffer $(5 \times), 2 \mu \mathrm{l}$ of dNTPs $(2.5 \mathrm{mM}), 1 \mu \mathrm{l}$ each of forward and reverse primer $(10 \mathrm{uM}), 2 \mu \mathrm{l}$ of DNA template, 0.25 $\mu \mathrm{l}$ of Q5 High-Fidelity DNA Polymerase $(5 \mathrm{U} / \mu \mathrm{l})$ and $8.75 \mu \mathrm{l}$ of $\mathrm{ddH}_{2} \mathrm{O}$. PCR conditions consisted of initial denaturation at $98^{\circ} \mathrm{C}$ for $5 \mathrm{~min}$, followed by 25 cycles of denaturation at $98^{\circ} \mathrm{C}$ for $30 \mathrm{~s}$, annealing at $52^{\circ} \mathrm{C}$ for $30 \mathrm{~s}$ and extension at $72^{\circ} \mathrm{C}$ for $1 \mathrm{~min}$, with a final extension at $72^{\circ} \mathrm{C}$ for $5 \mathrm{~min}$. PCR products were verified by $2 \%$ agarose gel electrophoresis and target fragments were purified using an AxyPrep gel extraction kit (Axygen, Union City, CA, USA ). The purified PCR products were then sequenced on an Illumina Miseq platform using $2 \times 300$ base pairs (bp) pairedend reads (Personalbio, Shanghai, China).

\section{Sequence analysis}

Sequencing data was processed using the Quantitative Insights Into Microbial Ecology (QIIME, v.1.8.0) pipeline as previously described (Caporaso et al., 2010). Resulting sequence with average Phred scores lower than 20, ambiguous bases, mononucleotide repeats longer than $8 \mathrm{bp}$, or those which had a length shorter than $100 \mathrm{bp}$ were removed. Sequences that passed the filter without any mismatches were trimmed and merged using FLASH v.1.2.11 (Magoc \& Salzberg, 2011), according to the principle of $98 \%$ overlap of 19 bases. The resulting sequences of each sample were clustered into operational taxonomic units (OTUs) based on $97 \%$ identity using the UCLUST function in QIIME (Caporaso et al., 2010; Edgar et al., 2010). OTU taxonomic classification was conducted using a BLAST search of the representative sequences set against the Greengenes database (Release 13.8, http://greengenes.secondgenome.com/) (DeSantis et al., 2006) and using the best hit (Altschul et al., 1997). An OTU table was generated to record the abundance and taxonomy of each OTU in each sample. OTUs with a sequence frequency below $0.001 \%$ were not included in the analysis (Bokulich et al., 2013). To minimize the difference of sequencing depth across samples, an averaged, rounded rarefied OTU table was generated by averaging 100 evenly resampled OTU subsets under the $90 \%$ of the minimum sequencing depth for further analysis.

\section{Bioinformatics analysis}

Rarefaction curves were calculated at a $97 \%$ similarity level in QIIME. Richness (Chaol and ACE) and diversity (Simpson and Shannon) indices were calculated for the microbiota using an out table in QIIME. Structural variation in the microbiota in the four parts of the midgut was investigated using Weighted UniFrac principal coordinate analysis (PCoA) in QIIME (Lozupone \& Knight, 2005; Lozupone et al., 2007). A maximum likelihood (ML) tree was constructed based on the Kimura 2-parameter model using MEGA X with 1000 bootstrap replicates (Kumar et al., 2018). The function of the gut microbiota was predicted using PICRUSt v1.1.3 Release and Greengenes as the sequenced reference, according to the online protocol (http://picrust.github. io/picrust/index.html) (DeSantis et al., 2006; McDonald et al., 2012; Langille et al., 2013). Firstly, OTUs which mapped to the GG13.5 database with a 97\% similarity were selected by QIIME. The OTUs counts were normalized by dividing by the $16 \mathrm{~S}$ rRNA gene copy numbers from known bacterial genomes in Integrated Microbial Genomes (IMG); normalized OTU tables were 

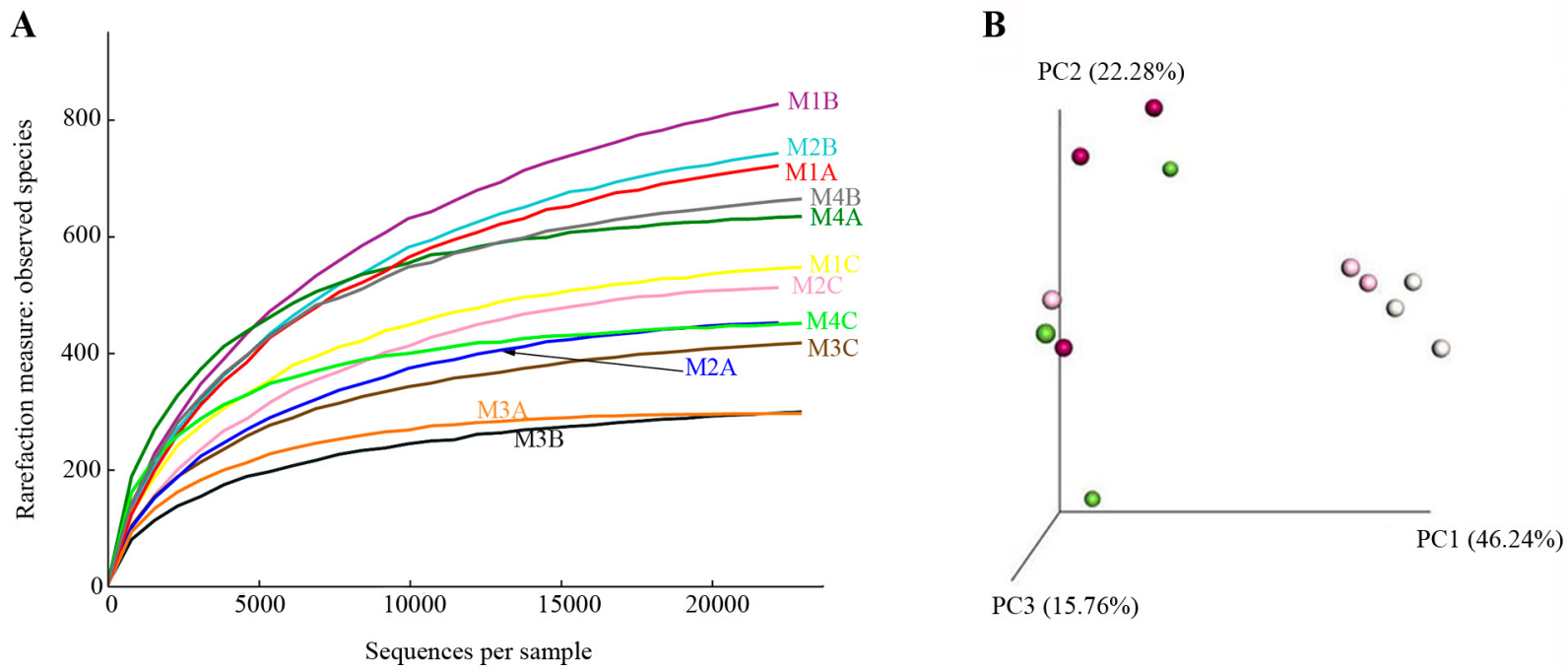

Fig. 2. Rarefaction curve of the samples (A) and PCA analysis of their microbiota (B) for each treatment.

then aligned to the Kyoto Encyclopedia of Genes and Genomes (KEGG) database. The nearest sequenced taxon index (NSTI) was calculated to quantify the quality of the predictions. Heatmap was generated using hemI (Deng et al., 2014). PCA analysis was conducted in STAMP v.2.1.3 (Parks et al., 2014) and contributions of core genera were generated from PICRUSt results using Excel. The functional differences between two-groups were compared using two-sided Welch's $t$-test in the Majorbio Cloud (cloud.majorbio.com).

\section{RESULTS}

The microbiota in the M1, M2, M3 and M4 of P. sibiricus were characterized using $16 \mathrm{~S}$ rRNA high-throughput sequencing. A total of 463,717 reads were generated from all of the independent biological treatments and replicates. The average amplicon length of the $16 \mathrm{~S}$ rRNA variable V3-V4 region was $420 \mathrm{bp}$. After quality filtering and read merging, 405,095 (87.36\%) sequences were obtained $(105,842,96,241,98,594$ and 104,418 sequences for M1, M2, M3 and M4, respectively). After assignment using UCLUST and removing OTUs with a relative abundance less than $0.001 \%$, a total of $1,111,1,060,496$ and 1,217 OTUs were obtained for M1, M2, M3 and M4, respectively. All taxa with relative abundances $>0.001 \%$ are cited in Table S3. Rarefaction curves indicate sufficient sequenc-

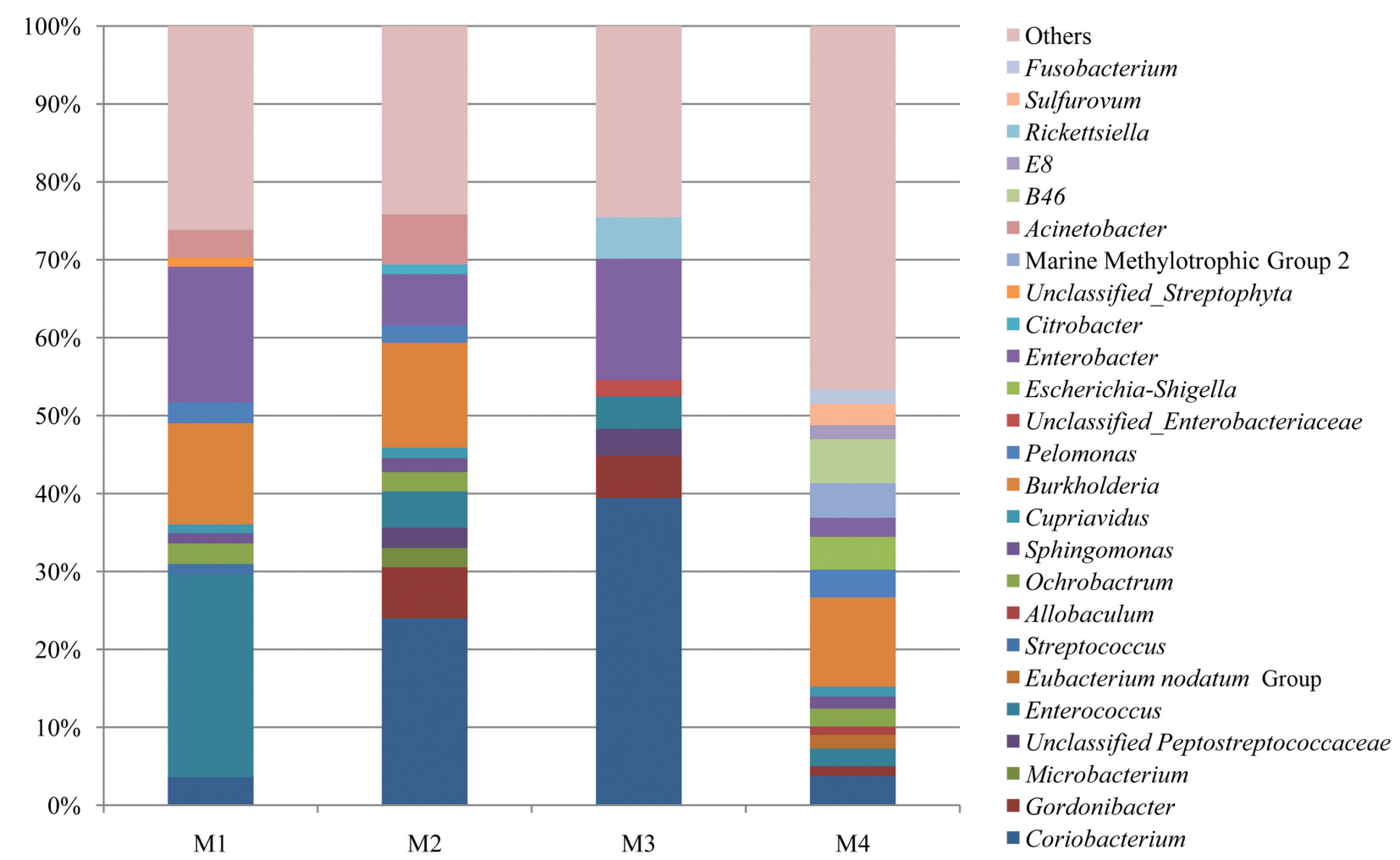

Fig. 3. Bacterial composition in terms of genera in the four parts of the midgut. Genera with an abundance lower than $1 \%$ are referred to as "Others". 


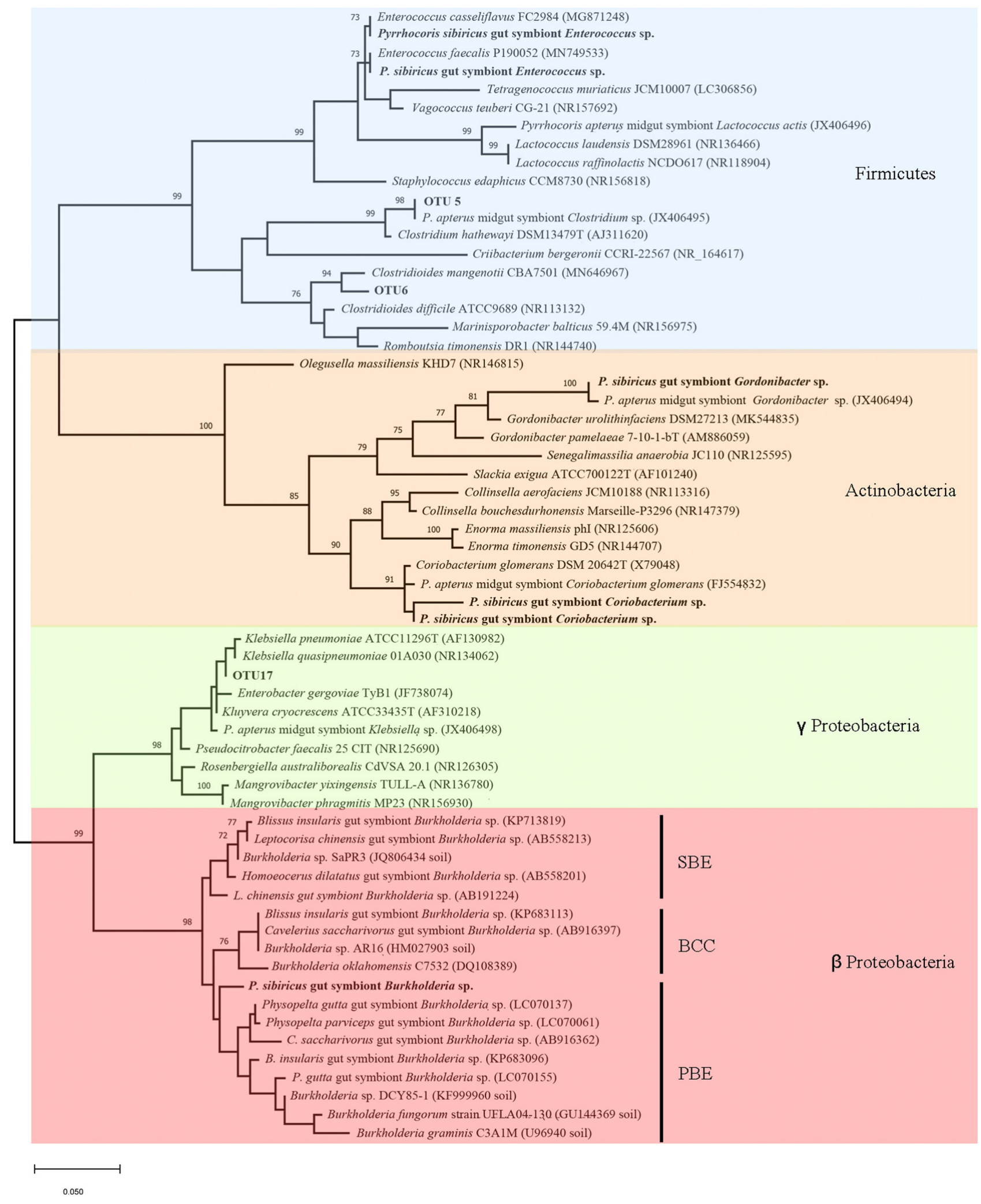

Fig. 4. Phylogenetic analysis based on the sequences of Coriobacterium sp., Gordonibacter sp., Enterococcus sp., Burkholderia sp. and unassigned OTUs in the different parts of the midgut of Pyrrhocoris sibiricus. Bootstrap values more than $70 \%$ are displayed on the bacterial phylogenies. Abbreviations of clades: BCC - Burkholderia cepacia complex; PBE - plant-associated beneficial and environmental group; SBE - stinkbug-associated beneficial and environmental group.

ing depth for taxonomic classification (Fig. 2A). For all samples, estimates of ACE richness indicate that M2 had the richest microbiota and the Shannon index suggests that diversity in M4 was significantly higher than in the other parts of the midgut (Table S1). 
A

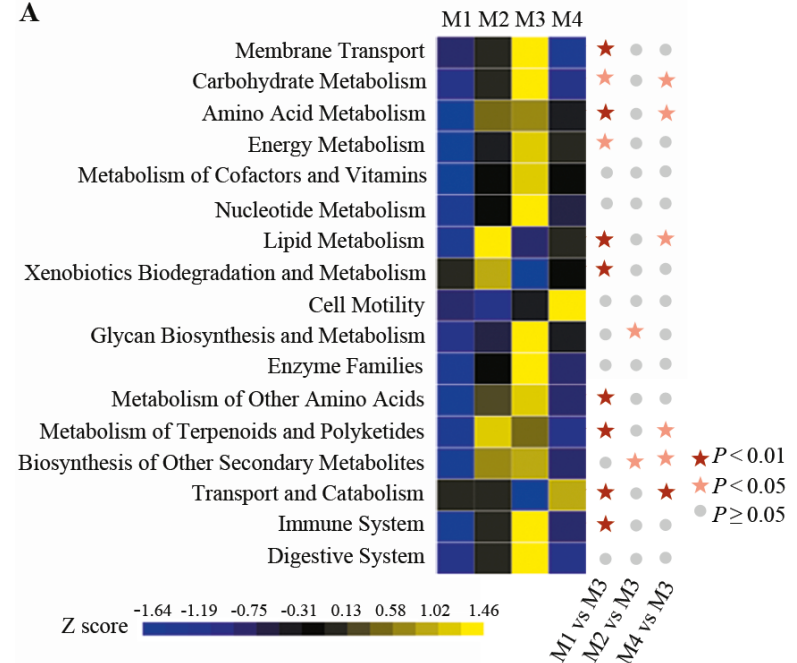

B

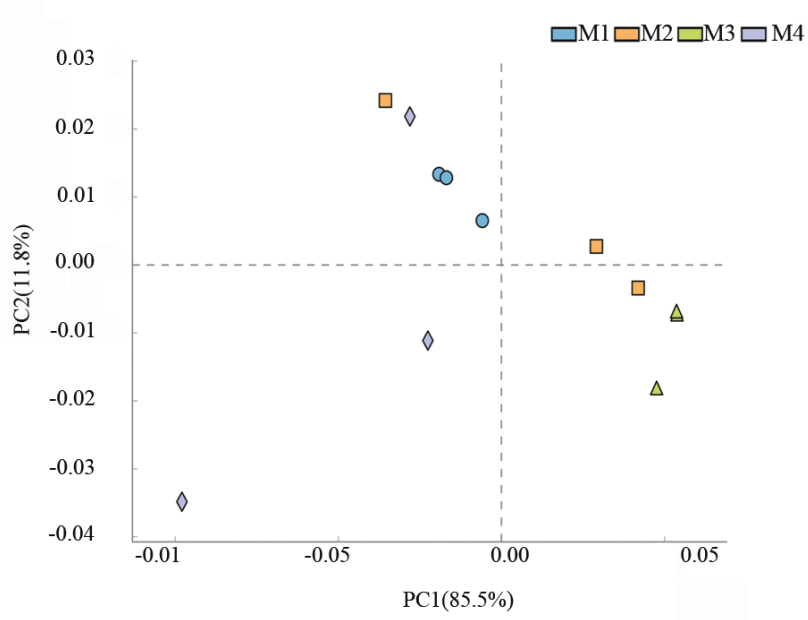

Fig. 5. Predicted KEGG pathways in different parts of the midgut of Pyrrhocoris sibiricus. A - heatmap of the KEGG modules differentially enriched in the different midgut microbiomes. KEGG pathways with a $P$-value $<0.01$ are marked with a dark red star, $<0.05$ with light red star and $\geq 0.05$ with a grey circle. B - PCA based on the relative abundance of KEGG orthology groups.

\section{Diversity of bacteria in gut microbiota}

At the phylum level Proteobacteria, Actinobacteria, Firmicutes and Bacteroidetes were present in all samples of the midgut of $P$. sibiricus. Proteobacteria was the most abundant in M1 (56.39\%), M2 (51.68\%) and M4 (68.31\%) followed by Firmicutes in M1 and M4 (31.60\% and $15.97 \%$, respectively). In M2, Actinobacteria was the second most abundant, accounting for $35.51 \%$ of all bacteria. The M3 region was unique in that Actinobacteria dominated followed by Proteobacteria with abundances of $49.58 \%$ and $37.11 \%$, respectively (Fig. S1). Principal component analysis revealed that M2 and M3 were most similar in terms of their microbiota (Fig. 2B).

When OTUs with an abundance lower than $1 \%$ were removed, 10, 13, 8 and 18 core genera were identified in M1, M2, M3 and M4, respectively (Fig. 3). All OTUs with abundances greater than $1 \%$ are cited in Table S2. For the phylum Proteobacteria, the most dominant bacterial group was the gammaproteobacterial genus Enterobacter, which was present throughout the midgut. An unassigned OTU is closely related to Klebsiella pneumoniae with a $99.17 \%$ similarity (AF130982, Fig. 4). The Betaproteobacterial genus Burkholderia was detected in M1, M2 and M4. According to the maximum likelihood (ML) tree, which was constructed based on the sequence obtained from nextgeneration sequencing as well as representative sequences of in- and outgroup taxa, the Burkholderia detected in the midgut of $P$. sibiricus closely matched sequences of the PBE clade (Fig. 4).

Within the phylum Actinobacteria, the genus Coriobacterium was recorded in all samples, mostly with high levels of relative abundance in M2 and M3. According to the ML phylogenetic tree, the two Coriobacterium OTUs were closely related to strains of Coriobacterium glomerans (X79048, FJ554832) that occur in the intestinal tract of $P$. apterus with $97.66 \% 16 \mathrm{~S}$ rRNA gene sequence similarity. A Gordonibacter OTU was identified in $P$. sibiricus that only had a $92.57 \% 16 \mathrm{~S}$ rDNA similarity with its clos- est relative, Gordonibacter pamelaeae (AM886059), and $99.26 \%$ with Gordonibacter sp. (JX406494) strain in the gut of $P$. apterus. This OTU was first clustered with Gordonibacter sp. with an overall mean genetic distance of 0.022 and then with cultured strains of the genus Gordonibacter (Fig. 4).

Members of the phylum Firmicutes also occurred in gut micribiota. One unassigned Firmicutes OTU was closely related to a strain in the gut of P. apterus (JX406495, with $98.03 \%$ similarity), one was clustered with a cultured strain of Clostridioides mangenotii (MN646967, with 97.28\% similarity) and the other two with Enterococcus faecalis (MN749533, with 98.47\% similarity) and E. casseliflavus (MG871248, with 98.08\% similarity) (Fig. 4).

\section{Predictive functional profiles}

In order to determine differences in the functions of the microbiota in the different parts of the midgut we carried out a functional analysis using PICRUSt. This revealed that 17 level2 KEGG orthology groups (KOs) differentially occurred in the four parts of the midgut, which is depicted in a heatmap (Fig. 5A). The mean NSTI values were $0.06,0.04,0.02$ and 0.08 for M1, M2, M3 and M4, respectively. Significant differences in predicted microbial functions (two-sided Welch's $t$-test, $P<0.05$ ) were detected between M3 and the other parts of the midgut (Fig. 5A, Table S4). PCA analysis showed that M2 and M3 have the most similar predicted microbial functions (Fig. 5B). Contributions of core genera to the predicted functions, which differ significantly among groups, are presented in Fig. 6. Enterobacter and Burkholderia contribute most to the predicted functions in M1, M2 and M4, while in M3 it is Enterobacter and Coriobacterium (Fig. 6).

\section{DISCUSSION}

In the present study, the microbiota inhabiting the midgut of $P$. sibiricus were characterized using $16 \mathrm{~S}$ rRNA metagenomic analysis. The results indicate that it differs 

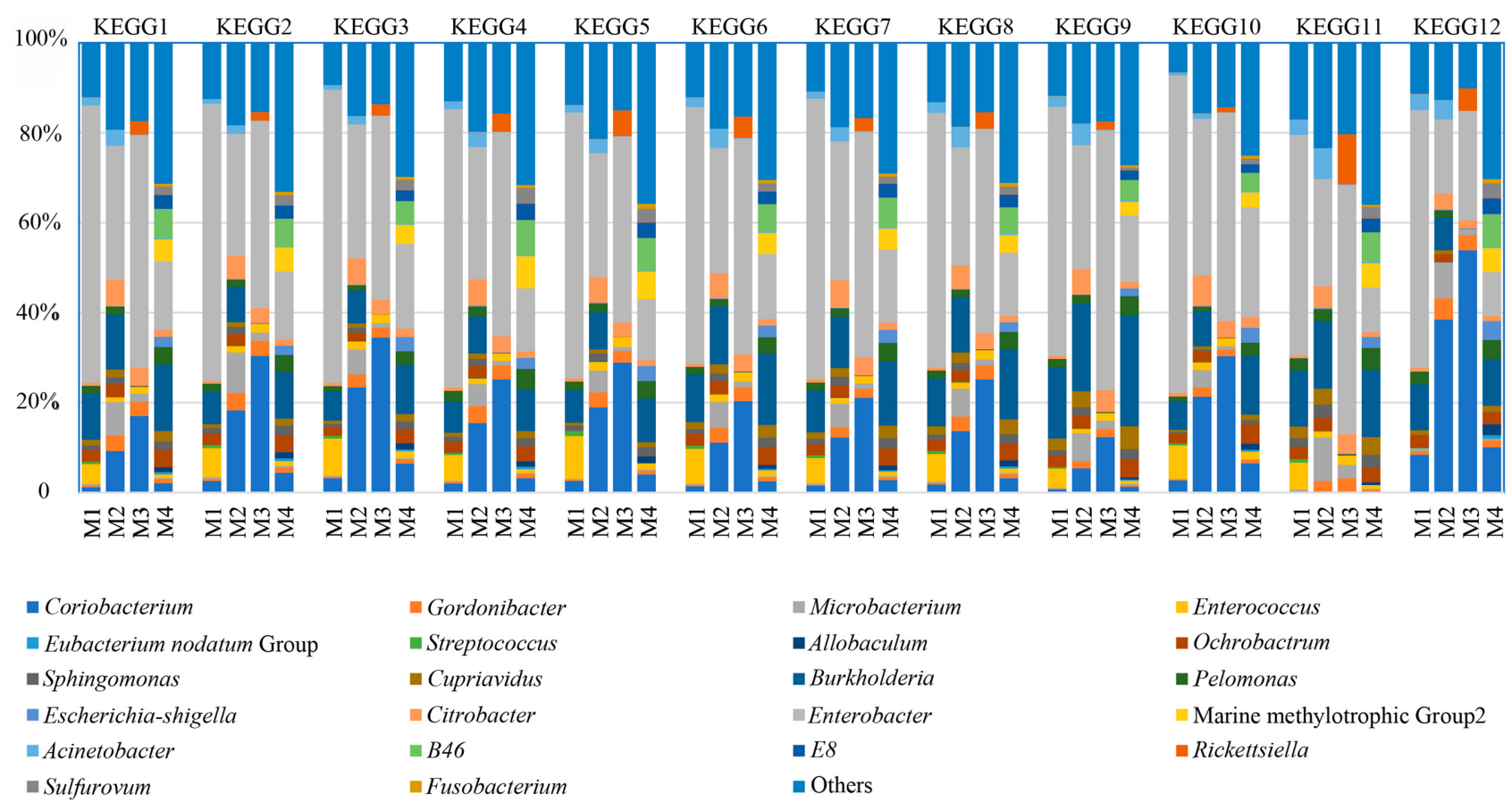

Fig. 6. Contributions of core genera to the predicted KEGG pathways. KEGG1-12: Amino acid metabolism; Biosynthesis of other secondary metabolites; Carbohydrate metabolism; Energy metabolism; Glycan Biosynthesis and Metabolism; Lipid metabolism; Metabolism of other amino acids; Metabolism of terpenoids and polyketides; Xenobiotic biodegradation and metabolism; Membrane transport; Transport and catabolism; Immune system.

in the different parts of the midgut (Fig. S1). Proteobacteria are the dominant bacteria in M1, M2 and M4. In M3, however, there is a distinct microbiota, consisting predominantly of Actinobacteria (Fig. S1). These results are in accord with previous studies on D. fasciatus and P. apterus (Sudakaran et al., 2012; Salem et al., 2013). The composition of the microbiota in M2 is particularly interesting in having a similar microbial profile of proteobacteria to M1 whilst sharing some core bacterial taxa with M3 (Fig. 2).

C. glomerans and Gordonibacter sp. (Actinobacteria), Clostridium sp. (Firmicutes) and Klebsiella sp. (Proteobacteria) occur in most species of Pyrrhocoridae (Salem et al., 2013; Sudakaran et al., 2015). Similarly, M3 in $P$. sibiricus is dominated by species of Coriobacterium and Gordonibacter, which are closely related to those recorded in P. apterus (C. glomerans and Gordonibacter sp., respectively; Figs 3,4$)$. In addition, there were also some OTUs with high sequence similarity with species of Clostridium and Klebsiella.

There were, however, some differences, the most notable being the distribution of Gordonibacter sp. and C. glomerans. In previous studies, they were recorded mainly in M3 (Sudakaran et al., 2012). However, they also make up a high proportion of the microbiota in M2. Furthermore, Lactococcus lactis and an undescribed Rickettsiales bacterium, which are core bacteria in M3 in P. apterus (Sudakaran et al., 2012), were absent in all our samples. We also recorded a relatively high abundance of Burkholderia and Enterobacter. Previously, Burkholderia was thought to be absent in Pyrrhocoridae, including Pyrrhocoris (Sudakaran et al., 2015), however, Gordon et al. (2016) report a high concentration of Burkholderia in the pyrrhocorid Euscopus rufipes. Hence, particular symbionts may be restricted to certain species of Pyrrhocoridae.

Species of Burkholderia are symbiotic partners of species of the superfamilies Lygaeoidea and Coreoidea, and particularly the Largidae in Pyrrhocoroidea (Kikuchi et al., 2011a; Itoh et al., 2014; Gordon et al., 2016). Generally, Burkholderia are acquired directly from the soil by early instar nymphs and stored in well-developed crypts in the posterior midgut (Kikuchi et al., 2011b; Boucias et al., 2012). However, in P. sibiricus, these symbionts are not only to the posterior midgut but also present in M1, M2 and M4. Early microscopic studies indicate that Burkholderia in Hemiptera belong to three distinct clades: the Burkholderia cepacia complex (BCC), the plant-associated beneficial and environmental (PBE) group, and the stinkbug-associated beneficial and environmental group (SBE) (Itoh et al., 2014). Unlike other Burkholderia associations in Alydidae (SBE clade) and Blissidae (all clades), the Burkholderia in P. sibiricus are closely related to the PBE clade (Itoh et al., 2014; Takeshita et al., 2015; Gordon et al., 2016). The function of Burkholderia symbionts in stinkbugs is unknown (Kaltenpoth \& Flórez, 2020). It is hypothesized (Takeshita et al., 2015) that they fix nitrogen for their insect hosts, however, this is still be tested experimentally. In this study, Burkholderia contributes a high proportion of the genes in the metagenome associated with xenobiotic biodegradation and metabolism than any of the other pathways studied. This may indicate that Burkholderia in $P$. sibiricus provides a secondary context-dependent benefit, namely resistance to insecticides, which is reported in the bean bug, Riptortus pedestris and oriental chinch bug, Cavelerius saccharivorus (Kikuchi et al., 2012). 
Many herbivorous insects have symbionts that supply nitrogen, essential amino acids, B vitamins and sterols, which are not present in the plant material they consume (Jones, 1984; Douglas, 1992, 1998). Seeds of Malvales are vitamin-limited and are generally avoided by other phytophagous bugs. Furthermore, phytochemical defences (gossypol and cyclopropenoic fatty acids) of the seeds interfere with digestion and so retarded growth, which can result in sterility (Allen et al., 1967; Abou-Donia, 1976; Kristenová et al., 2011). In previous studies, symbionts of Coriobacteriaceae are reported to activate food polyphenols and dietary phytoestrogens (Clavel et al., 2014; Chew et al., 2018). Some species, such as C. glomerans, can supply B vitamins to the host and protect them from parasites (Salem et al., 2014; Onchuru et al., 2018) and species of Clostridia play a role in the fermentation of carbohydrates, including the degradation of cellulose (Makonde et al., 2013; Sabree \& Moran, 2014). Enterobacter belonging to the Enterobacteriaceae are present in the gut of Mediterranean fruit flies (Ceratitis capitate) (Aharon et al., 2013; Kyritsis et al., 2019). They are important in nitrogen and carbon metabolism and used as a dietary supplement (probiotic) in the diets to rear their larvae (Behar et al., 2005, 2008; Ben-Yosef et al., 2008; Augustinos et al., 2015; Kyritsis et al., 2019). Our study revealed that Enterobacter, Coriobacterium and Burkholderia make up a big proportion of the bacteria and contribute to most of the functions. The heatmap of the metabolic processes occurring in the different parts of the midgut reveals that M2 and M3 are involved in carbohydrate, amino acid, energy, lipid and vitamin metabolism, which accords with our $16 \mathrm{~S}$ rRNA metagenomic results, which also indicate that these parts of the midgut are the main sites for food digestion, but the mechanism needs further study.

With the help of bacterial symbionts, Hemiptera are able to exploit plant tissues, such as xylem and phloem, which are nutrient-limited and contain phytochemicals (Buchner, 1965). In recent decades, our knowledge of Hemiptera and their microbes has increased considerably, particularly the identification of their core microbiota, genome sequencing, phylogenetic relationships, and the role of symbionts in the provision of nutrients, insecticide-resistance and defence against parasites (Kikuchi et al., 2012; Stackebrandt et al., 2013; Kaiwa et al., 2014; Hosokawa et al., 2016; Sudakaran et al., 2017; Onchuru et al., 2018; Onchuru \& Kaltenpoth, 2019; Kaltenpoth \& Flórez, 2020). However, few studies have focused on the role symbionts in the digestion of food. Although further study is needed to verify the exact mechanism, our results indicate that the microbiota in the M2 and M3 parts of the midgut play an important role in degrading complex dietary components.

ACKNOWLEDGEMENTS. This work was supported by the National Natural Science Foundation of China (31501840), Scientific and Technologial Innovation Programs of Higher Education Institutions in Shanxi (2019L0803), Biodiversity Survey and Assessment Project of the Ministry of Ecology and Environment in China (2019HJ2096001006), and Doctoral scientific research foundation of Taiyuan Normal University.

\section{REFERENCES}

ABou-Donia M.B. 1976: Physiological effects and metabolism of gossypol. — Residue Rev. 61: 125-160.

Aharon Y., Pasternak Z., Ben Yosef M., Behar A., Lauzon C., Yuval B. \& Jurkevitch E. 2013: Phylogenetic, metabolic, and taxonomic diversities shape mediterranean fruit fly microbiotas during ontogeny. - Appl. Environ. Microbiol. 79: 303-313.

Ahmad I. \& Schaefer C.W. 1987: Food plants and feeding biology of the Pyrrhocoroidea (Hemiptera). - Phytophaga 1: 75-92.

Akman L., Yamashita A., Watanabe H., Oshima K., Shiba T., Hattori M. \& Aksoy S. 2002: Genome sequence of the endocellular obligate symbiont of tsetse flies, Wigglesworthia glossinidia. - Nat. Genet. 32: 402-407.

Allen E., Johnson A.R., Fogerty A.C., Pearson J.A. \& ShenSTONE F.S. 1967: Inhibition by cyclopropene fatty acids of the desaturation of stearic acid in hen liver. - Lipids 2: 419-423.

Altschul S.F., Madden T.L., Schaffer A.A., Zhang J.H., Zhang Z., Miller W. \& Lipman D.J. 1997: Gapped BLAST and PSIBLAST: a new generation of protein database search programs. - Nucl. Acids Res. 25: 3389-3402.

Augustinos A.A., Kyritsis G.A., Papadopoulos N.T., Abd-Alla A.M.M., CÁceres C. \& Bourtzis K. 2015: Exploitation of the medfly gut microbiota for the enhancement of sterile insect technique: use of Enterobacter sp. in larval diet-based probiotic applications. — PLOS ONE 10(9): e 0136459, 17 pp.

Behar A., Yuval B. \& Jurkevitch E. 2005: Enterobacteria-mediated nitrogen fixation in natural populations of the fruit fly Ceratitis capitata. - Mol. Ecol. 14: 2637-2643.

Behar A., Yuval B. \& Jurkevitch E. 2008: Gut bacterial communities in the Mediterranean fruit fly (Ceratitis capitata) and their impact on host longevity. - J. Insect Physiol. 54: 1377-1383.

Ben-Yosef M., Jurkevitch E. \& Yuval B. 2008: Effect of bacteria on nutritional status and reproductive success of the Mediterranean fruit fly Ceratitis capitata. - Physiol. Entomol. 33: $145-154$.

Bokulich N.A., Subramanian S., Faith J.J., Gevers D., Gordon J.I., Knight R., Mills D.A. \& CAporaso J.G. 2013: Qualityfiltering vastly improves diversity estimates from Illumina amplicon sequencing. - Nat. Meth. 10: 57-59.

Boucias D.G., Garcia-Maruniak A., Cherry R., Lu H., MaruNIAK J.E. \& LieTZE V.U. 2012: Detection and characterization of bacterial symbionts in the Heteropteran, Blissus insularis. - FEMS Microbiol. Ecol. 82: 629-641.

BuchNER P. 1965: Endosymbiosis of Animals with Plant Microorganisms. Interscience Publishers, New York, 909 pp.

Caporaso J.G., Kuczynski J., Stombaugh J., Bittinger K., Bushman F.D., Costello E.K., Fierer N., Pena A.G., Goodrich J.K., GoRdon J.I. ET AL. 2010: QIIME allows analysis of highthroughput community sequencing data. - Nat. Meth. 7: 335336.

Chew Y.M., Lye S.F., Salleh M.M. \& Yahya A. 2018: 16S rRNA metagenomic analysis of the symbiotic community structures of bacteria in foregut, midgut, and hindgut of the wood-feeding termite Bulbitermes sp. - Symbiosis 76: 187-197.

Clavel T., Lepage P. \& Charrier C. 2014: The Family Coriobacteriaceae. In Rosenberg E., Delong E., Thompson F., Lory S. \& Stackebrandt E. (eds): The Prokaryotes. 4th ed. Springer, Heidelberg, pp. 201-238.

Deng W., Wang Y., Liu Z., Cheng H. \& Xue Y. 2014: HemI: A toolkit for illustrating heatmaps. - PLOS ONE 9(11): e111988, 5 pp. 
DeSantis T.Z., Hugenholtz P., Larsen N., Rojas M., Brodie E.L., Keller K., Huber T., Dalevi D., Hu P. \& Andersen G.L. 2006: Greengenes, a chimera-checked 16S rRNA gene database and workbench compatible with ARB. - Appl. Environ. Microbiol. 72: 5069-5072.

Douglas A.E. 1992: Microbial brokers of insect-plant interactions. In Menken S.B.J., Visser J.H. \& Harrewijn P. (eds): Proceedings of the 8th International Symposium on Insect-Plant Relationships. Series Entomologica 49. Springer, Dordrecht, pp. 329-336.

Douglas A.E. 1998: Nutritional interactions in insect-microbial symbioses: aphids and their symbiotic bacteria Buchnera. Annu. Rev. Entomol. 43: 17-37.

EdGAR R.C. 2010: Search and clustering orders of magnitude faster than BLAST. - Bioinformatics 26: 2460-2461.

Genta F.A., Dillon R.J., Terra W.R. \& Ferreira C. 2006: Potential role for gut microbiota in cell wall digestion and glucoside detoxification in Tenebrio molitor larvae. - J. Insect Physiol. 52: 593-601.

Gordon E.R.L., McFrederick Q. \& Weirauch C. 2016: Phylogenetic evidence for ancient and persistent environmental symbiont reacquisition in Largidae (Hemiptera: Heteroptera). Appl. Environ. Microbiol. 82: 7123-7133.

HenRY T.J. 2009: Biodiversity of the Heteroptera. In Foottit R.G. \& Adler P.H. (eds): Insect Biodiversity: Science and Society. Wiley-Blackwell, Oxford, pp. 223-263.

Hosokawa T., Ishi Y., Nikoh N., Fujie M., Satoh N. \& Fukatsu T. 2016: Obligate bacterial mutualists evolving from environmental bacteria in natural insect populations. - Nat. Microbiol. 1: 15011, 7 pp.

Itoh H., Aita M., Nagayama A., Meng X.Y., Kamagata Y., Navarro R., Hori T., OhgiYa S. \& KiKUChI Y. 2014: Evidence of environmental and vertical transmission of Burkholderia symbionts in the oriental chinch bug, Cavelerius saccharivorus (Heteroptera: Blissidae). - Appl. Environ. Microbiol. 80: 5974-5983.

JoNES C.G. 1984: Microorganisms as mediators of plant resource exploitation by insect herbivores. In Price P.W., Slobodchikoff C.N. \& Gaud W.S. (eds): A New Ecology: Novel Approaches to Interactive Systems. Wiley, New York, pp. 53-99.

Kaiwa N., Hosokawa T., Nikoh N., Tanahashi M., Moriyama M., Meng X.Y., Maeda T., Yamaguchi K., Shigenob S., Ito M. ET AL. 2014: Symbiont-supplemented maternal investment underpinning host's ecological adaptation. - Curr. Biol. 24: $2465-2470$

KaltenPoth M. \& FlóRez L.V. 2020: Versatile and dynamic symbioses between insects and Burkholderia bacteria. - Annu. Rev. Entomol. 65: 7.1-7.26.

KikUchi Y., Hosokawa T. \& FuKatsu T. 2011a: Specific developmental window for establishment of an insect-microbe gut symbiosis. - Appl. Environ. Microbiol. 77: 4075-4081.

KikUchi Y., Hosokawa T. \& FuKatsu T. 2011b: An ancient but promiscuous host-symbiont association between Burkholderia gut symbionts and their heteropteran hosts. - ISME J. 5: 446-460.

Kikuchi Y., Hayatsu M., Hosokawa T., Nagayama A., Tago K. \& FUKATSU T. 2012: Symbiont-mediated insecticide resistance. PNAS 109: 8618-8622.

KristenovÁ M., Exnerová A. \& ŠTYs P. 2011: Seed preferences of Pyrrhocoris apterus (Heteroptera: Pyrrhocoridae): are there specialized trophic populations? - Eur. J. Entomol. 108: 581586.

Kumar S., Stecher G., Li M., Knyaz C. \& Tamura K. 2018: MEGA X: Molecular evolutionary genetics analysis across computing platforms. - Mol. Biol. Evol. 35: 1547-1549.
Kyritsis G.A., Augustinos A.A., Ntougias S., Papadopoulos N.T., Bourtzis K. \& CÁceres C. 2019: Enterobacter sp. AA26 gut symbiont as a protein source for Mediterranean fruit fly mass-rearing and sterile insect technique applications. - BMC Microbiol. 19(S1): 288, 15 pp.

Langille M.G.I., Zaneveld J., Caporaso J.G., McDonald D., Knights D., Reyes J.A., Clemente J.C., Burkepile D.E., Thurber R.L.V., Knight R. et AL. 2013: Predictive functional profiling of microbial communities using 16S rRNA marker gene sequences. - Nat. Biotechnol. 31: 814-821.

LozUPone C. \& KNight R. 2005: UniFrac: a new phylogenetic method for comparing microbial communities. - Appl. Environ. Microbiol. 71: 8228-8235.

Lozupone C.A., Hamady M., Kelley S.T. \& Knight R. 2007: Quantitative and qualitative beta diversity measures lead to different insights into factors that structure microbial communities. - Appl. Environ. Microbiol. 73: 1576-1585.

MAGOC T. \& SAlzBerg S.L. 2011: FLASH: fast length adjustment of short reads to improve genome assemblies. - Bioinformatics 27: 2957-2963.

Makonde H.M., Boga H.I., Osiemo Z., Mwirichia R., Mackenzie L.M., GOKERM. \& KLENK H. 2013: 16S-rRNA-based analysis of bacterial diversity in the gut of fungus-cultivating termites (Microtermes and Odontotermes species). - Anton. Leeuw. 104: 869-883.

Matolin S. \& S̆TYS P. 1987: Embryonic inviability in reciprocal crosses between Pyrrhocoris apterus and P. sibiricus (Heteroptera, Pyrrhocoridae). — Acta Entomol. Bohemoslov. 84: 246-253.

McDonald D., Price M.N., Goodrich J., Nawrocki E.P., DeSantis T.Z., Probst A., Andersen G.L., Knight R. \& Hugenholtz P. 2012: An improved Greengenes taxonomy with explicit ranks for ecological and evolutionary analyses of bacteria and archaea. - ISME J. 6: 610-618.

Mizrahi-Man O., Davenport E.R. \& Gilad Y. 2013: Taxonomic classification of bacterial $16 \mathrm{~S}$ rRNA genes using short sequencing reads: evaluation of effective study designs. - PLOS ONE 8: e53608, $14 \mathrm{pp}$.

Moran N.A., Mccutcheon J.P. \& NaKabachi A. 2008: Genomics and evolution of heritable bacterial symbionts. - Annu. Rev. Genet. 42: 165-190.

OnChURU T.O. \& KALTENPOTH M. 2019: Established cotton stainer gut bacterial mutualists evade regulation by host antimicrobial peptides. - Appl. Environ. Microbiol. 85: e00738-19, 14 pp.

Onchuru T.O., Martinez A.J. \& KaltenPoth M. 2018: The cotton stainer's gut microbiota suppresses infection of a cotransmitted trypanosomatid parasite. - Mol. Ecol. 27: 3408-3419.

Parks D.H., Tyson G.W., Hugenholtz P. \& Beiko R.G. 2014: STAMP: statistical analysis of taxonomic and functional profiles. - Bioinformatic 30: 3123-3124.

SABreE Z.L. \& Moran N.A. 2014: Host-specific assemblages typify gut microbial communities of related insect species. SpringerPlus 3: 138, 11 pp.

Salem H., Kreutzer E., Sudakaran S. \& Kaltenpoth M. 2013: Actinobacteria as essential symbionts in firebugs and cotton stainers (Hemiptera, Pyrrhocoridae). - Environ. Microbiol. 15: 1956-1968.

Salem H., Bauer E., Strauss A.S., Vogel H., Marz M. \& KALTENPOTH M. 2014: Vitamin supplementation by gut symbionts ensures metabolic homeostasis in an insect host. - Proc. Roy. Soc. (B) 281: 20141838, 10 pp.

SAXENA K.N. 1963: Mode of ingestion in a heteropterous insect Dysdercus koenigii (F.) (Pyrrhocoridae). - J. Insect Physiol. 9: 47-71. 
Schaefer C.W. \& Ahmad I. 2000: Cotton stainers and their relatives (Pyrrhocoroidea: Pyrrhocoridae and Largidae). In Schaefer C.W. \& Panizzi A.R. (eds): Heteroptera of Economic Importance. CRC Press, Boca Raton, pp. 271-307.

Silva C.P. \& TerRa W.R. 1994: Digestive and absorptive sites along the midgut of the cotton seed sucker bug Dysdercus peruvianus (Hemiptera: Pyrrhocoridae). - Insect Biochem. Mol. Biol. 24: 493-505.

Sмітн J.M. 1989: Generating novelty by symbiosis. — Nature 341: 284-285.

Stackebrandt E., Zeytun A., Lapidus A., Nolan M., Lucas S., Hammon N., Deshrande S., Cheng J.F., Tapia R., Goodwin L.A. ET AL. 2013: Complete genome sequence of Coriobacterium glomeranstype strain $\left(\mathrm{PW}^{\mathrm{T}}\right)$ from the midgut of Pyrrhocoris apterus L. (red soldier bug). - Stand. Genomic Sci. 8: 15-25.

Sudakaran S., Salem H., Kost C. \& Kaltenpoth M. 2012: Geographical and ecological stability of the symbiotic mid-gut microbiota in European firebugs, Pyrrhocoris apterus (Hemiptera, Pyrrhocoridae). - Mol. Ecol. 21: 6134-6151.

Sudakaran S., Retz F., Kikuchi Y., Kost C. \& Kaltenpoth M. 2015: Evolutionary transition in symbiotic syndromes enabled diversification of phytophagous insects on an imbalanced diet. - ISME J. 9: 2587-2604.

Sudakaran S., Kost C. \& Kaltenpoth M. 2017: Symbiont acquisition and replacement as a source of ecological innovation. - Trends Microbiol. 25: 375-390.

Takeshita K., Matsuura Y., Itoh H., Navarro R., Hori T., Sone T., Kamagata Y., Mergaert P. \& KIKUChI Y. 2015: Burkholderia of plant-beneficial group are symbiotically associated with bordered plant bugs (Heteroptera: Pyrrhocoroidea: Largidae). - Microbes Environ. 30: 321-329.
Taylor C.M., Coffey P.L., DeLay B.D. \& Dively G.P. 2014: The importance of gut symbionts in the development of the brown marmorated stink bug, Halyomorpha halys (Stål). — PLoS ONE 9(3): e90312, 9 pp.

Zhang S.M. 1985: Economic Insect Fauna of China. Fasc. 31, Hemiptera (1). Science Press, Beijing, 165 pp.

Received March 21, 2020; revised and accepted August 26, 2020 Published online September 24, 2020

Supplementary material follows (Tables S1-S4, Fig. S1).

Table S1. 16S rRNA gene sequence reads and alpha-diversity indices recorded in the different treatments.

\begin{tabular}{lccccccc}
\hline \multirow{2}{*}{ Sample } & \multirow{2}{*}{$\begin{array}{c}\text { High } \\
\text { quality }\end{array}$} & \multirow{2}{*}{$\begin{array}{c}\text { No. of } \\
\text { modified }\end{array}$} & \multicolumn{2}{c}{ Richness indices } & & \multicolumn{2}{c}{ Diversity indices } \\
\cline { 8 - 9 } \cline { 7 - 8 } & sequence & OTUs & Chao1 & ACE & & Shannon & Simpson \\
\hline M1A & 36317 & 32192 & 792.57 & 813.99 & & 0.814697 & 4.32 \\
M1B & 41975 & 36801 & 972.58 & 998.37 & & 0.810928 & 4.52 \\
M1C & 37358 & 31019 & 568.45 & 584.64 & & 0.797064 & 4.42 \\
M2A & 36515 & 30417 & 457.38 & 469.54 & & 0.848671 & 4.27 \\
M2B & 39843 & 32413 & 839.93 & 849.29 & & 0.854713 & 4.77 \\
M2C & 32096 & 29578 & 515.07 & 523.25 & & 0.892772 & 4.68 \\
M3A & 33453 & 25624 & 297 & 297 & & 0.802357 & 4.06 \\
M3B & 42176 & 33720 & 340.92 & 338.49 & & 0.829836 & 4.03 \\
M3C & 48319 & 37869 & 446.99 & 454.83 & & 0.845618 & 4.49 \\
M4A & 37697 & 31176 & 647.13 & 652.57 & & 0.966306 & 6.53 \\
M4B & 37213 & 34148 & 734.12 & 712.98 & & 0.896785 & 5.23 \\
M4C & 40755 & 34544 & 490.33 & 471.62 & & 0.950102 & 5.99
\end{tabular}

The "No. of modified OTUs" means the clean OTUs with abundance $>0.001 \%$.

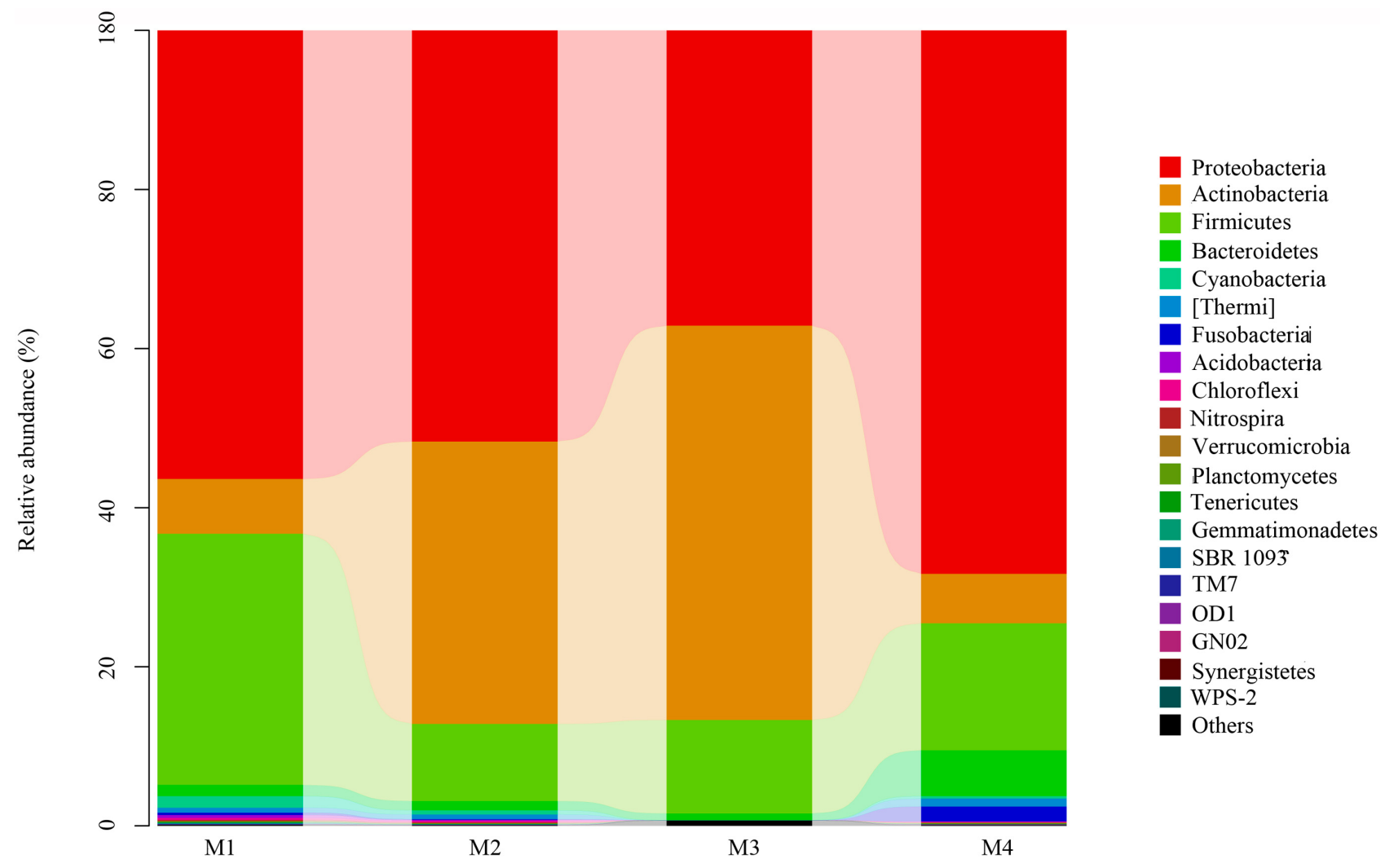

Fig. S1. Bacterial composition in terms of phyla in the four parts of the midgut. 


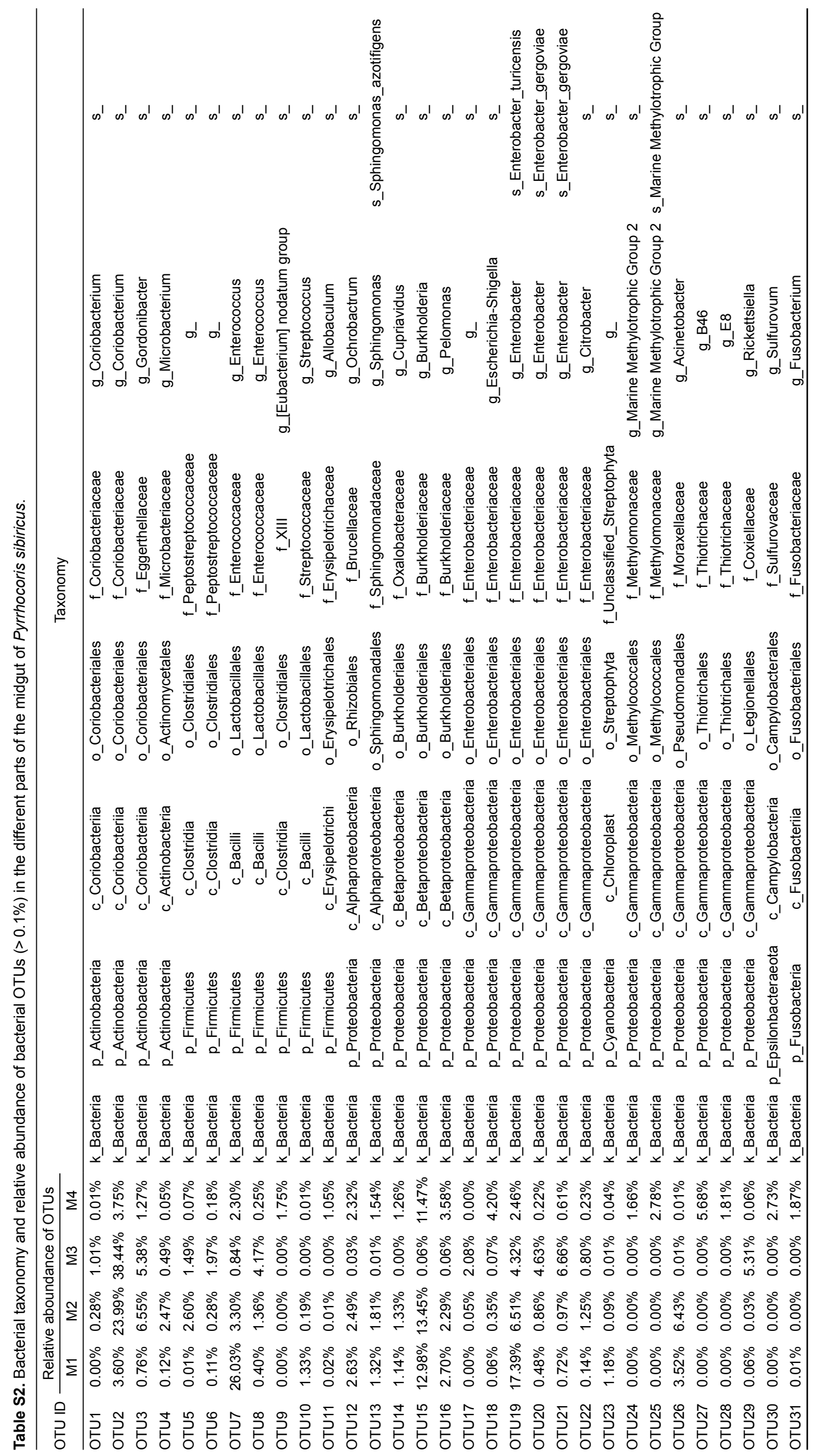


Table S3. All taxa with a relative abundance $>0.001 \%$ recorded in the different parts of the midgut of Pyrrhocoris sibiricus

Taxonomy

\begin{tabular}{|c|c|c|c|c|c|c|c|c|c|}
\hline & & & & & & M1 & M2 & M3 & M4 \\
\hline p_[Thermi] & C_Deinococci & o_Deinococcales & f_Trueperaceae & g_B-42 & s_Unclassified_B-42 & $0.02 \%$ & $0.01 \%$ & $0.00 \%$ & $0.00 \%$ \\
\hline $\mathrm{p} \_[\text {Thermi] }$ & c_Deinococci & o_Deinococcales & f_Trueperaceae & $\begin{array}{l}\text { g_Unclassified_ } \\
\text { Trueperaceae }\end{array}$ & $\begin{array}{l}\text { S_Unclassified_- } \\
\text { Trueperaceae }\end{array}$ & $0.01 \%$ & $0.00 \%$ & $0.00 \%$ & $0.08 \%$ \\
\hline p_[Thermi] & C_Deinococci & o_Thermales & f_Thermaceae & g_Thermus & s_Unclassified_Thermus & $0.60 \%$ & $0.59 \%$ & $0.00 \%$ & $0.93 \%$ \\
\hline k_Bacteria p_Acidobacteria & c_[Chloracidobacteria] & o_RB41 & f_Ellin6075 & $\begin{array}{c}\text { g_Unclassified_ } \\
\text { Ellin6075 }\end{array}$ & $\begin{array}{l}\text { ssified__ } \\
075\end{array}$ & $0.00 \%$ & $0.00 \%$ & $0.00 \%$ & $0.00 \%$ \\
\hline k_Bacteria p_Acidobacteria & c_[Chloracidobacteria] & O_RB & Unclassifi & g_Unclassified_RB41 & d_RB41 & $0.00 \%$ & $0.01 \%$ & $0.00 \%$ & $0.01 \%$ \\
\hline k_Bacteria p_Acidobacteria & c_Acidobacteria-5 & $\begin{array}{l}\text { O_Unclassified_ } \\
\text { Acidobacteria-5 }\end{array}$ & $\begin{array}{l}\text { f_Unclassified- } \\
\text { Acidobacteria-5 }\end{array}$ & $\begin{array}{l}\text { g_Unclassified- } \\
\text { Acidobacteria- } 5\end{array}$ & & $0.01 \%$ & $0.00 \%$ & $0.00 \%$ & $0.00 \%$ \\
\hline Bacteria $\mathrm{p} \_$Acidobacteria & c_Acidobacteria-6 & o_BPC015 & Unclassified_BPC015 & g_Unclassified_BPC015 & d_BPC015 & $0.01 \%$ & $0.00 \%$ & $0.00 \%$ & $0.00 \%$ \\
\hline Bacteria p_Acidobacteria & C_Acido & & Unclas & _Unclassified_CC & 21 & $0.02 \%$ & $0.02 \%$ & $0.00 \%$ & $0.00 \%$ \\
\hline Bacteria p_Acidot & c Acido & & & Unclass & $\mathrm{mb} 2424$ & $0.00 \%$ & $0.00 \%$ & $0.00 \%$ & $0.00 \%$ \\
\hline Bacteria p_Acidot & C_Aci & & Uncla & g_Unclas: & d_iii1-15 & $0.05 \%$ & $0.02 \%$ & $0.00 \%$ & $0.01 \%$ \\
\hline k_Bacteria p_Acidobacteria & C_Acidobacteriia & Acidobacteriales & f_Acido & & rosea & $0.00 \%$ & $0.00 \%$ & $0.00 \%$ & $0.00 \%$ \\
\hline k_Bacteria p_Acidobacteria & c_Acidobacteriia & O_Acidobacteriales & f_Koribacteraceae & $\begin{array}{l}\text { g_Unclassified_ } \\
\text { Koribacteraceae }\end{array}$ & & $0.01 \%$ & $0.00 \%$ & $0.00 \%$ & $0.00 \%$ \\
\hline _Bacteria p_Acidobacteria & c_AT-s2-57 & $\begin{array}{c}\text { O_Unclassified_AT- } \\
\text { s2-57 }\end{array}$ & f_Unclassified_AT-s2-57 & g_Unclassified_AT-s2-57 & 7 s_Unclassified_AT-s2-57 & $0.01 \%$ & $0.01 \%$ & $0.00 \%$ & $0.00 \%$ \\
\hline Bacteria $\mathrm{p} \_$Acidobacteria & C_BPC102 & & f_Unclassified_B110 & g_Unclassified_B110 & S_Unclassified_B110 & $0.05 \%$ & $0.02 \%$ & $0.00 \%$ & $0.00 \%$ \\
\hline Bacteria p_Acidobacteria & c_BP & & f_Unclas & g_Unclass & MVS-40 & $0.00 \%$ & $0.00 \%$ & $0.00 \%$ & $0.01 \%$ \\
\hline Bacteria p_Acidobacteria & & Uncla & f_Uncl & d_OS-K & OS-K & $0.03 \%$ & $0.00 \%$ & $0.00 \%$ & $0.00 \%$ \\
\hline k_Bacteria p_Acidobacteria & 25 & Unclassi & f_Unclass & RB25 & RB25 & $0.03 \%$ & $0.01 \%$ & $0.00 \%$ & $0.00 \%$ \\
\hline Bacteria $\mathrm{p} \_$Acidobacteria & c_Solibacteres & o_Solibacterales & f_PAUC26f & $\begin{aligned} \text { g_Un } \\
\text { Pf }\end{aligned}$ & s_Unclassified_PAUC26f & $f 0.01 \%$ & $0.01 \%$ & $0.00 \%$ & $0.00 \%$ \\
\hline Bacteria p_Acidobacteria & c_Solibacteres & o_Solibacterales & f_Solibacteraceae & $\begin{array}{r}\text { g_Ca } \\
\text { So }\end{array}$ & $\begin{array}{c}\text { S_Unclassified__ } \\
\text { Candidatus Solibacter }\end{array}$ & $0.00 \%$ & $0.00 \%$ & $0.00 \%$ & $0.00 \%$ \\
\hline K_Bacteria p_Acidobacteria & c_Solibacteres & o_Solibacterales & f_Solibacteraceae & $\begin{array}{l}\text { g_Un } \\
\text { Soliba }\end{array}$ & & $0.01 \%$ & $0.00 \%$ & $0.00 \%$ & $0.00 \%$ \\
\hline Bacteria p_Acidobacteria & c_Solibacteres & o_Solibacterales & $\begin{array}{l}\text { f_Unc } \\
\text { Solib }\end{array}$ & $\begin{array}{l}\text { g_Unc } \\
\text { Solib: }\end{array}$ & les & $0.01 \%$ & $0.01 \%$ & $0.00^{\circ}$ & $0.01 \%$ \\
\hline Bacteria p_Acidobacteria & & o_Sv & Unclassified_Sva072 & g_Unclassified_Sva07 & Sva0725 & $50.13 \%$ & $0.03 \%$ & $0.00 \%$ & $0.00 \%$ \\
\hline k_Bacteria p_Acidobacteria & $\begin{array}{l}\text { C_Uncla } \\
\text { Acidob }\end{array}$ & $\begin{array}{l}\text { O_Unc } \\
\text { Acido }\end{array}$ & $\begin{array}{l}\text { f_Unc } \\
\text { Acido }\end{array}$ & $\begin{array}{l}\text { g_Un } \\
\text { Acid }\end{array}$ & & $0.00 \%$ & $0.01 \%$ & $0.00^{\circ}$ & $0.00 \%$ \\
\hline Bacteria p_Actinobacteria & c_Acidimicrobiia & o_Acidim & & g_Uncle & $\mathrm{C} 111$ & $0.03 \%$ & $0.01 \%$ & $0.00 \%$ & $0.02 \%$ \\
\hline k_Bacteria p_Actinobacteria & c_Acidimicrobiia & o_Acidimicrobiales & f_JdFBGBact & $\begin{array}{l}\text { g_Un } \\
\text { JdF }\end{array}$ & ed_- & $0.00 \%$ & $0.00 \%$ & $0.00 \%$ & $0.29 \%$ \\
\hline Bacteria p_Actinobacteria & c_Acidimicrobiia & o_Acidim & f_koll13 & koll13 & koll13 & $0.18 \%$ & $0.09 \%$ & $0.00 \%$ & $0.00 \%$ \\
\hline a_Actino & oiia & Aci & & Uncla & ntu14 & $0.00 \%$ & $0.00 \%$ & $0.00 \%$ & $0.00 \%$ \\
\hline Bacteria p_Actinobacteria & c_Acidin & Acidi & & g_Uncle & TK06 & $0.00 \%$ & $0.01 \%$ & $0.00 \%$ & $0.00 \%$ \\
\hline k_Bacteria p_Actinobacteria & c_Acidimicrobiia & o_Acidimicrobiales & $\begin{array}{l}\text { f_Unc } \\
\text { Acidim }\end{array}$ & & & $0.13 \%$ & $0.09 \%$ & $0.00 \%$ & $0.03 \%$ \\
\hline k_Bacteria $\mathrm{p} \_$Actinobacteria & c_Acidimicrobiia & o_Acidimicrobiales & f_wb1_P06 & & s_un & $0.05 \%$ & $0.03 \%$ & $0.00 \%$ & $0.00 \%$ \\
\hline _Bacteria p_Actinobacteria & c_Actinobacteria & o_Actinomycetales & f_Actinomycetaceae & g_Actinomyces & & $0.39 \%$ & $0.05 \%$ & $0.00 \%$ & $0.00 \%$ \\
\hline Bacteria p_Actinobacteria & c_Actinobacteria & Actino & f_Actinos & g_Lentzea & Lentzea & $0.00 \%$ & $0.00 \%$ & $0.00 \%$ & $0.00 \%$ \\
\hline k_Bacteria p_Actinobacteria & c_Actinobacteria & o_Actinomycetales & f_Brevibacteriaceae & g_Brevibacterium & & $0.03 \%$ & $0.02 \%$ & $0.00 \%$ & $0.02 \%$ \\
\hline Bacteria p_Actinobacteria & c_Actinobacteria & o_Actinomycetales & f_Cellulomonadaceae & g_Cellulomonas & & $0.00 \%$ & $0.00 \%$ & $0.00^{\circ}$ & $0.00 \%$ \\
\hline Bacteria p_Actinobacteria & c_Actinobacteria & o_Actinomycetales & f_Corynebacteriaceae & g_Coryneba & $\mathrm{S}$ & $0.14 \%$ & $0.24 \%$ & $0.00^{\circ}$ & $0.09 \%$ \\
\hline __Bacteria p_Actinobacteria & c_Actinobacteria & o_Actinomycetales & f_Cryptosporangiaceae & $\begin{array}{c}\text { 9_Unclassified_ } \\
\text { Cryptosporangiaceae }\end{array}$ & $\mathrm{d}_{-}$ & $0.00 \%$ & $0.01 \%$ & $0.00 \%$ & $0.00 \%$ \\
\hline Bacteria p_Actinobacteria & c_Actinobacteria & o_Actinomycetales & f_Dermabacteraceae & g_Brachybacterium & & $0.00 \%$ & $0.00 \%$ & $0.00^{\circ}$ & $0.01 \%$ \\
\hline k_Bacteria p_Actinobacteria & c_Actinobacteria & o_Actinomycetales & f_Dermabacteraceae & g_Brachybacterium & & $0.00 \%$ & $0.00 \%$ & $0.00 \%$ & $0.00 \%$ \\
\hline Bacteria p_Actinobacteria & c_Actinobacteria & o_Actinomycetales & f_Dietziaceae & & Dietzia & $0.01 \%$ & $0.00 \%$ & $0.00 \%$ & $0.01 \%$ \\
\hline k_Bacteria p_Actinobacteria & c_Actinobacteria & o_Actinomycetales & Geodermatophilaceae & $\begin{array}{l}\text { g_Unclassified__ } \\
\text { Geodermatophilaceae }\end{array}$ & d- & $0.01 \%$ & $0.00 \%$ & $0.00 \%$ & $0.00 \%$ \\
\hline K_Bacteria p_Actinobacteria & c_Actinobacteria & o_Actinomycetales & f_Gordoniaceae & g_Gordonia & & $0.00 \%$ & $0.00 \%$ & $0.00 \%$ & $0.00 \%$ \\
\hline Bacteria p_Actinobacteria & c_Actinobacteria & o_Actinomycetales & f_Intrasporangiaceae & g_K & & $0.00 \%$ & $0.00 \%$ & $0.00 \%$ & $0.04 \%$ \\
\hline Bacteria p_Actinobacteria & c_Actinobacteria & o_Actinomycetales & f_Intrasporangiaceae & $\begin{array}{l}\text { g_Unclassified_ } \\
\text { Intrasporangiaceae }\end{array}$ & ceare & $0.01 \%$ & $0.00^{\circ}$ & 0.00 & 0.01 \\
\hline k_Bacteria p_Actinobacteria & c_Actinobacteria & o_Actinomycetales & f_Microbacteriaceae & g_Agromyces & & $0.00 \%$ & $0.00 \%$ & $0.00 \%$ & $0.00 \%$ \\
\hline Bacteria p_Actinobacteria & c_Actinobacteria & o_Actino & f_Micro & & Cryocola & $0.01 \%$ & $0.04 \%$ & $0.00^{\circ}$ & $0.01 \%$ \\
\hline k_Bacteria $p \_$Actinobacteria & c_Actinobacteria & o_Actinomycetales & f_Microbacteriaceae & g_Curtobacterium & & $0.00 \%$ & $0.00 \%$ & $0.01 \%$ & $0.00 \%$ \\
\hline k_Bacteria p_Actinobacteria & c_Actinobacteria & o_Actinomycetales & f_Microbacteriaceae & g_Microbacterium & & $0.12 \%$ & & $0.54 \%$ & $0.06 \%$ \\
\hline Bacteria p_Actinobacteria & c_Actinobacteria & o_Actinomycetales & f_Microbacteriaceae & g_M & & $0.00 \%$ & $0.00 \%$ & 0.00 & $0.00 \%$ \\
\hline Bacteria p_Actinobacteria & c_Actinobacteria & & & $\begin{array}{l}\text { g_Unc } \\
\text { Microba }\end{array}$ & & $0.00 \%$ & & & $0.00 \%$ \\
\hline c_Bacteria $p \_$Actinobacteria & c_Actinobacteria & o_Actinomycetales & f_Micrococcaceae & g_Arthrobacter & & $0.01 \%$ & $0.06 \%$ & $0.05 \%$ & $0.02 \%$ \\
\hline Bacteria p_Actinobacteria & c_Actinobacteria & o_Actinomycetales & f_Micrococcaceae & g_Micrococcus & & $0.01 \%$ & $0.02 \%$ & $0.00 \%$ & $0.03 \%$ \\
\hline Bacteria $\mathrm{p} \_$_Actinobacteria & c. Actinoba & Act & Mirr & & & & & & $0.00 \%$ \\
\hline Bacteria $\mathrm{p}$ _Actinc & c_Ac & & & & & & & $0 \%$ & $0.00 \%$ \\
\hline k_Bacteria $\mathrm{p} \_$_Actinobacteria & C_Actinobacteria & o_Actinomycetales & f_Micrococcaceae & & & $0.00 \%$ & $0.01 \%$ & $0.00 \%$ & $0.00 \%$ \\
\hline k_Bacteria p_Actinobacteria & c_Actinobacteria & o_Actinomycetales & f_Micrococcaceae & & & $0.00 \%$ & $0.00 \%$ & $0.00^{\circ}$ & $0.00 \%$ \\
\hline k_Bacteria p_Actinobacteria & c_Actinobacteria & o_Actinomycetales & __vilctormonsosporacede & g_Micromonospora & & $0.00 \%$ & $0.00 \%$ & $0.00^{\circ}$ & $0.00 \%$ \\
\hline k_Bacteria p_Actinobacteria & c_Actinobacteria & o_Actinomycetales & f_Micromonosporaceae & g_Phytohabitans & & $0.01 \%$ & $0.00 \%$ & 0.00 & 0.00 \\
\hline k_Bacteria p_Actinobacteria & c_Actinobacteria & o_Actinomycetales & f_Micromonosporaceae & g_Virgisporangium & ium_ & $0.00 \%$ & $0.01 \%$ & $0.00^{\circ}$ & $0.00 \%$ \\
\hline k_Bacteria p_Actinobacteria & c_Actinobacteria & o_Actinomycetales & f_Mycobacteriaceae & g_Mycobacterium & & $0.01 \%$ & $0.00 \%$ & 0.00 & $0.01 \%$ \\
\hline _Bacteria p_Actinobacteria & c_Actinobacteria & o_Actinomycetales & f_Mycobacteriaceae & g_Mycobacterium & & $0.01 \%$ & & & \\
\hline _Bacteria $\mathrm{p} \_$_Actinobacteria & c_Actinobacteria & o_Actinomycetales & f_Nocardiaceae & g_Rhodococcus & & $0.01 \%$ & $0.00 \%$ & $0.00^{\circ}$ & $0.00 \%$ \\
\hline Bacteria p_Actinobacteria & c_Actinobacteria & o_Actinomycetales & f_Nocardioidaceae & g_Aeromicrobium & & $0.00 \%$ & $0.00 \%$ & & $0.00 \%$ \\
\hline
\end{tabular}


Table S3 (continued)

Taxonomy

\begin{tabular}{|c|c|c|c|c|c|c|c|c|c|c|}
\hline Bacteria & Actinobacteria & C_Actinobacteria & Actinomycetales & f_Nocardioidaceae & g_Nocardioides & $\begin{array}{l}\text { S_Unclassified_ }_{\text {Nocardioides }} \\
\text { Nocis }\end{array}$ & $0.00 \%$ & $0.00 \%$ & $0.00 \%$ & $0.00 \%$ \\
\hline k_Bacteria & p_Actinobacteria & c_Actinobacteria & o_Actinomycetales & f_Nocardioidaceae & $\begin{array}{l}\text { g_Unclassified_ } \\
\text { Nocardioidaceae }\end{array}$ & $\begin{array}{l}\text { S_Unclassified } \\
\text { Nocardioidaceae }\end{array}$ & $0.01 \%$ & $0.01 \%$ & $0.00 \%$ & $0.00 \%$ \\
\hline k_Bacteria & p_Actinobacteria & c_Actinobacteria & o_Actinomycetales & f_Propionibacteriaceae & g_Propionibacterium & $\begin{array}{c}\text { S_Propionibacterium_ } \\
\text { acnes }\end{array}$ & $0.05 \%$ & $0.05 \%$ & $0.00 \%$ & $0.07 \%$ \\
\hline k_Bacteria & p_Actinobacteria & c_Actinobacteria & o_Actinomycetales & f_Propionibacteriaceae & g_Propionibacterium & s_Propionibacterium_ & $0.00 \%$ & $0.01 \%$ & $0.00 \%$ & $0.01 \%$ \\
\hline k_Bacteria & p_Actinobacteria & c_Actinobacteria & o_Actinomycetales & f_Pseudonocardiaceae & g_Amycolatopsis & osis & $0.13 \%$ & $0.11 \%$ & $0.00 \%$ & $0.12 \%$ \\
\hline k_Bacteria & p_Actinobacteria & c_Actinobacteria & o_Actinomycetales & f_Sanguibacteraceae & g_Sanguibacter & s_Sanguibacter_soli & $0.01 \%$ & $0.00 \%$ & $0.00 \%$ & $0.00 \%$ \\
\hline k_Bacteria & p_Actinobacteria & c_Actinobacteria & o_Actinomycetales & f_Sporichthyaceae & $\begin{array}{l}\text { g_Unclassified } \\
\text { Sporichthyaceae }\end{array}$ & $\begin{array}{l}\text { S_Unclassified- } \\
\text { Sporichthyaceae }\end{array}$ & $0.00 \%$ & $0.00 \%$ & $0.00 \%$ & $0.01 \%$ \\
\hline k_Bacteria & Actinobacteria & c_Actinobacteria & O_Actinomycetales & f_Streptomycetaceae & g_Streptomyces & s_Streptomyces_lanatus & $0.00 \%$ & $0.00 \%$ & $0.00 \%$ & $0.00 \%$ \\
\hline Bacteria & Actinobacteria & c_Actinobacteria & o_Actinomycetales & f_Streptomycetaceae & g_Streptomyces & $\begin{array}{c}\text { s_Streptomyces_ } \\
\text { mashuensis }\end{array}$ & $0.00 \%$ & $0.00 \%$ & $0.00 \%$ & $0.00 \%$ \\
\hline k_Bacteria & p_Actinobacteria & c_Actinobacteria & o_Actinomycetales & f_Streptomycetaceae & $\begin{array}{c}\text { g_Unclassified_- } \\
\text { Streptomycetaceae }\end{array}$ & $\begin{array}{c}\text { S_Unclassified } \\
\text { Streptomycetaceae }\end{array}$ & $0.00 \%$ & $0.00 \%$ & $0.00 \%$ & $0.01 \%$ \\
\hline Bacteria & p_Actinobacteria & c_Actinobacteria & o_Actinomycetales & Thermomonosp & g_Actinomadura & s_Actinomadura_vinacea & $0.00 \%$ & $0.01 \%$ & $0.00 \%$ & $0.00 \%$ \\
\hline Bacteria & p_Actinobacteria & c_Actinobacteria & o_Actinomycetales & $\begin{array}{c}\text { fUnclassified } \\
\text { Actinomycetales }\end{array}$ & $\begin{array}{l}\text { g_Unclassified_ } \\
\text { Actinomycetales }\end{array}$ & $\begin{array}{l}\text { S_Unclassified_- } \\
\text { Actinomycetales }\end{array}$ & $0.00 \%$ & $0.01 \%$ & $0.00 \%$ & $0.02 \%$ \\
\hline k_Bacteria & P_Actinobacteria & c_Actinobacteria & o_Bifidobacteriales & f_Bifidobacteriaceae & g_Bifidobacterium & & $0.00 \%$ & $0.00 \%$ & $0.00 \%$ & $0.02 \%$ \\
\hline Bacteria & Actinobacteria & c_Actinobacteria & o_Bifidobacteriales & f_Bifidobacteriaceae & g_Bifidobacterium & $\begin{array}{l}\text { s_Bifidobacterium_ } \\
\text { pseudolongum }\end{array}$ & $0.02 \%$ & $0.01 \%$ & $0.00 \%$ & $0.05 \%$ \\
\hline k_Bacteria & p_Actinobacteria & c_Actinobacteria & o_Bifidobacteriales & f_Bifidobacteriaceae & g_Gardnerella & $\begin{array}{l}\text { S_Unclassified_ } \\
\text { Gardnerella }\end{array}$ & $0.00 \%$ & $0.01 \%$ & $0.00 \%$ & $0.00 \%$ \\
\hline Bacteria & p_Actinobacteria & c_Actinobacteria & O_WCHB1-81 & f_At425_EubF1 & $\begin{array}{l}\text { g_Unclassified_ } \\
\text { At425_EubF1 }\end{array}$ & $\begin{array}{c}\text { S_Unclassified_At425_ } \\
\text { EubF1 }\end{array}$ & $0.00 \%$ & $0.00 \%$ & $0.00 \%$ & $0.00 \%$ \\
\hline k_Bacteria & p_Actinobacteria & c_Coriobacteriia & o_Coriobacteriales & f_Coriobacteriaceae & g_Adlercreutzia & $\begin{array}{l}\text { s_Unclassified__ } \\
\text { Adlercreutzia }\end{array}$ & $0.00 \%$ & $0.00 \%$ & $0.00 \%$ & $0.02 \%$ \\
\hline k_Bacteria & p_Actinobacteria & c_Coriobacteriia & o_Coriobacteriales & f_Coriobacteriaceae & g_Atopobium & s_Unclassified_Atopobium & $0.02 \%$ & $0.00 \%$ & $0.00 \%$ & $0.00 \%$ \\
\hline k_Bacteria & p_Actinobacteria & c_Coriobacteriia & o_Coriobacteriales & f_Coriobacteriaceae & g_Collinsella & s_Collinsella_aerofaciens & $0.00 \%$ & $0.00 \%$ & $0.00 \%$ & $0.00 \%$ \\
\hline k_Bacteria & p_Actinobacteria & c_Coriobacteriia & o_Coriobacteriales & f_Coriobacteriaceae & g_Coriobacterium & $\begin{array}{l}\text { S_Unclassified_- } \\
\text { Coriobacterium }\end{array}$ & $3.60 \%$ & $24.31 \%$ & $39.61 \%$ & $3.76 \%$ \\
\hline k_Bacteria & p_Actinobacteria & c_Coriobacteriia & o_Coriobacteriales & f_Eggerthellaceae & g_Gordonibacter & $\begin{array}{l}\text { s_Unclassified_- } \\
\text { Gordonibacter }\end{array}$ & $0.76 \%$ & $6.55 \%$ & $5.38 \%$ & $1.27 \%$ \\
\hline Bacteria & p_Actinobacteria & c_Coriobacteriia & o_Coriobacteriales & f_Coriobacteriaceae & $\begin{array}{l}\text { g_U } \\
\text { Coriol } \\
\end{array}$ & $\begin{array}{c}\text { S_Unclassified_ } \\
\text { Coriobacteriaceae }\end{array}$ & $0.07 \%$ & $0.97 \%$ & $3.97 \%$ & $0.10 \%$ \\
\hline k_Bacteria & p_Actinobacteria & C_MB-A2-108 & $\begin{array}{c}\text { O_Unclassified_ } \\
\text { MB-A2-108 }\end{array}$ & $\begin{array}{c}\text { f_Unclassified_MB- } \\
\text { A2-108 }\end{array}$ & g_Un & $\begin{array}{c}\text { S_Unclassified_MB-A2- } \\
108\end{array}$ & $0.00 \%$ & $0.01 \%$ & $0.00 \%$ & $0.00 \%$ \\
\hline Bacteria & p_Actinobacteria & C_Nitriliruptoria & o_Nitriliruptorales & f_Nitriliruptoraceae & $\begin{array}{l}\text { g_l } \\
\text { Nitril }\end{array}$ & $\begin{array}{l}\text { S_Unclassified_ } \\
\text { Nitriliruptoraceae }\end{array}$ & $0.00 \%$ & $0.01 \%$ & $0.00 \%$ & $0.01 \%$ \\
\hline Bacteria & p_Actinobacteria & c_OPB41 & $\begin{array}{c}\text { O_Unclassified_ } \\
\text { OPB41 }\end{array}$ & f_Unclassified_OPB41 & $\begin{array}{c}\text { g_Unclassified }_{-} \\
\text {OPB41 }\end{array}$ & s_Unclassified_OPB41 & $0.00 \%$ & $0.00 \%$ & $0.00 \%$ & $0.00 \%$ \\
\hline k_Bacteria & p_Actinobacteria & c_Rubrobacteria & O_Rubrobacterales & f_Rubrobacteraceae & g_Rubrobacter & $\begin{array}{l}\text { Sified_ } \\
\text { acter }\end{array}$ & $0.00 \%$ & $0.00 \%$ & $0.00 \%$ & $0.01 \%$ \\
\hline Bacteria & p_Actinobacteria & c_Thermoleophilia & o_Gaiellales & f_Gaiellaceae & $\begin{array}{l}\text { ified_ } \\
\text { iae }\end{array}$ & $\begin{array}{l}\text { s_Unclassified_ } \\
\text { Gaiellaceae }\end{array}$ & $0.04 \%$ & $0.04 \%$ & $0.00 \%$ & $0.04 \%$ \\
\hline k_Bacteria & P_Actinobacteria & C_Thermoleophilia & o_Gaiellales & $\begin{array}{c}\text { f_Unclassified_ } \\
\text { Gaiellales }\end{array}$ & g_t & s_Unclassified_Gaiellales & $0.00 \%$ & $0.00 \%$ & $0.00 \%$ & $0.00 \%$ \\
\hline Bacteria & p_Actinobacteria & c_Thermoleophilia & Solirubrobacterales & f_Patulibacteraceae & $\begin{array}{l}\text { g_Unclassified_ } \\
\text { Patulibacteraceae }\end{array}$ & sified_ & $0.00 \%$ & $0.00 \%$ & $0.00 \%$ & $0.01 \%$ \\
\hline k_Bacteria & P_Actinobacteria & c_Thermoleophilia & $\stackrel{O_{-}}{\text {Solirubrobacterales }}$ & f_Solirubrobacteraceae & g_Solirubrobacter & ssified_- & $0.00 \%$ & $0.01 \%$ & $0.00 \%$ & $0.00 \%$ \\
\hline Bacteria & p_Actinobacteria & c_Thermoleophilia & Solirubrobacterales & f_Solirubrobacteraceae & $\begin{array}{l}\text { g_Unclassified_- } \\
\text { Solirubrobacteraceae }\end{array}$ & $\begin{array}{l}\text { S_Unclassified_- } \\
\text { Solirubrobacteraceae }\end{array}$ & $0.00 \%$ & $0.00 \%$ & $0.00 \%$ & $0.00 \%$ \\
\hline Bacteria & p_AD3 & c_ABS-6 & $\begin{array}{c}\text { O_Unclassified_ } \\
\text { ABS-6 }\end{array}$ & f_Unclassified_ABS-6 & g_Unclassified_ABS-6 & s_Unclassified_ABS-6 & $0.00 \%$ & $0.01 \%$ & $0.00 \%$ & $0.01 \%$ \\
\hline __Bacteria & $\begin{array}{c}\mathrm{p}_{-} \\
\text {Armatimonadetes }\end{array}$ & c_[Fimbriimonadia] & $\begin{array}{c}\text { [E- } \\
\text { [Fimbriimonadales] }\end{array}$ & f_[Fimbriimonadaceae] & g_Fimbriimonas & ified_- & $0.00 \%$ & $0.01 \%$ & $0.00 \%$ & $0.00 \%$ \\
\hline Bacteria & $\begin{array}{c}\mathrm{p}_{-} \\
\text {Armatimonadetes } \\
\end{array}$ & $\begin{array}{c}\mathrm{C}_{-} \\
\text {Chthonomonadetes }\end{array}$ & $\begin{array}{c}0_{-} \\
\text {Chthonomonadales }\end{array}$ & f_Chthonomonadaceae & g_Chthonomonas & nomonas & $0.00 \%$ & $0.00 \%$ & $0.00 \%$ & $0.00 \%$ \\
\hline _Bacteria & p_Bacteroidetes & c_[Rhodothermi] & $\begin{array}{c}\mathrm{O}_{-} \\
\text {[Rhodothermales] }\end{array}$ & f_Rhodothermaceae & $\begin{array}{c}\text { g_Unclassified_ } \\
\text { Rhodothermaceae }\end{array}$ & $\begin{array}{l}\text { lassified } \\
\text { lermaceae } \\
\text { dassified }\end{array}$ & $0.02 \%$ & $0.01 \%$ & $0.00 \%$ & $0.00 \%$ \\
\hline Bacteria & p_Bacteroidetes & c_[Saprospirae] & o_[Saprospirales] & f_Chitinophagaceae & g_Sediminibacterium & & $0.52 \%$ & $0.73 \%$ & $0.00 \%$ & $0.17 \%$ \\
\hline Bacteria & p_Bacteroidetes & c_[Saprospirae] & o_[Saprospirales] & f_Saprospiraceae & & $\begin{array}{l}\text { S_Unclassified_ } \\
\text { Saprospiraceae }\end{array}$ & $0.07 \%$ & $0.02 \%$ & $0.00 \%$ & $0.15 \%$ \\
\hline Bacteria & p_Bacteroidetes & C_[Saprospirae] & o_[Saprospirales] & $\begin{array}{l}\text { f_Unclassified_ } \\
\text { [STaprospirales] }\end{array}$ & & $\begin{array}{l}\text { S_Unclassified_ } \\
\text { [Saprospirales] }\end{array}$ & $0.01 \%$ & $0.00 \%$ & $0.00 \%$ & $0.01 \%$ \\
\hline Bacteria & p_Bacteroidetes & Bacteroidia & o_Bacteroidales & f_[Barnesiellaceae] & & & $0.00 \%$ & $0.00 \%$ & $0.00 \%$ & $0.02 \%$ \\
\hline K_Bacteria & p_Bacteroidetes & C_Bacteroidia & o_Bacteroidales & f_[Paraprevotellaceae] & g_Para & & $0.00 \%$ & $0.00 \%$ & $0.00 \%$ & $0.09 \%$ \\
\hline k_Bacteria & p_Bacteroidetes & c_Bacteroidia & O_Bacteroidales & f_Bacteroidaceae & g_Bacteroides & S_Bacteroides_fragilis & $0.00 \%$ & $0.01 \%$ & $0.00 \%$ & $0.00 \%$ \\
\hline k_Bacteria & p_Bacteroidetes & Bacteroidia & O_Bacteroidales & f_Bacteroidaceae & g_Bacteroides & s_Bacteroides_ovatus & $0.00 \%$ & $0.01 \%$ & $0.00 \%$ & $0.00 \%$ \\
\hline k_Bacteria & p_Bacteroidetes & Bacteroidia & O_Bacteroidales & f_Bacteroidaceae & g_Bacteroides & s_Bacteroides_plebeius & $0.01 \%$ & $0.00 \%$ & $0.00 \%$ & $0.00 \%$ \\
\hline k_Bacteria & p_Bacteroidetes & c_Bacteroidia & o_Bacteroidales & f_Bacteroidaceae & g_Bacteroides & s_Bacte & $0.00 \%$ & $0.00 \%$ & $0.00 \%$ & $0.01 \%$ \\
\hline k_Bacteria & p_Bacteroidetes & c_Bacteroidia & o_Bacteroidales & f_Bacteroidaceae & g_Bacteroides & & $0.02 \%$ & $0.03 \%$ & $0.00 \%$ & $1.12 \%$ \\
\hline k_Bacteria & p_Bacteroidetes & Bacteroidia & o_Bacteroidales & f BS11 & g_Unclassified_BS11 & s_Uncl & $0.04 \%$ & $0.02 \%$ & $0.00 \%$ & $0.00 \%$ \\
\hline k_Bacteria & p_Bacteroidetes & c_Bacteroidia & O_Bacteroidales & f_Porphyromonadaceae & g_Dysgonomonas & s_Dysgo & $0.00 \%$ & $0.00 \%$ & $0.83 \%$ & $0.00 \%$ \\
\hline k_Bacteria & p_Bacteroidetes & c_Bacteroidia & o_Bacteroidales & f_Porphyromonadaceae & g_Dysgonomonas & $\begin{array}{l}\text { S_ } \\
\text { Dys }\end{array}$ & $0.00 \%$ & $0.01 \%$ & $0.01 \%$ & $0.00 \%$ \\
\hline k_Bacteria & p_Bacteroidetes & c_Bacteroidia & o_Bacteroidales & f_Porphyromonadaceae & g_Dysgonomonas & & $0.00 \%$ & $0.00 \%$ & $0.01 \%$ & $0.00 \%$ \\
\hline k_Bacteria & p_Bacteroidetes & C_Bacteroidia & o_Bacteroidales & f_Porphyromonadaceae & g_Parabacteroides & & $0.00 \%$ & $0.00 \%$ & $0.00 \%$ & $0.12 \%$ \\
\hline Bacteria & p_Bacteroidetes & c_Bacteroidia & o_Bacteroidales & f_Porphyromonadaceae & g_Parabacteroides & 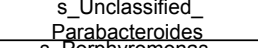 & $0.00 \%$ & $0.00 \%$ & $0.00 \%$ & $0.00 \%$ \\
\hline k_Bacteria & p_Bacteroidetes & c_Bacteroidia & o_Bacteroidales & f_Porphyromonadaceae & g_Porphyromonas & $\begin{array}{r}\mathrm{s}_{-} \mathrm{P} \\
\mathrm{e}\end{array}$ & $0.00 \%$ & $0.00 \%$ & $0.00 \%$ & $0.00 \%$ \\
\hline Bacteria & p_Bacteroidetes & c_Bacteroidia & o_Bacteroidales & f_Porphyromonadaceae & g_Porphyromonas & & $0.05 \%$ & $0.01 \%$ & $0.00 \%$ & $0.00 \%$ \\
\hline k_Bacteria & p_Bacteroidetes & c_Bacteroidia & o_Bacteroidales & f_Porphyromonadaceae & $\begin{array}{l}\text { g_Unclassified } \\
\text { Porphyromonadacea }\end{array}$ & $\begin{array}{l}\text { S_U } \\
\text { Porphy }\end{array}$ & $0.00 \%$ & $0.00 \%$ & $0.00 \%$ & $0.00 \%$ \\
\hline k_Bacteria & p_Bacteroidetes & c_Bacteroidia & O_Bacteroidales & f_Prevotellaceae & g_Prevotella & S_Prev & $0.00 \%$ & $0.00 \%$ & $0.00 \%$ & $0.00 \%$ \\
\hline k_Bacteria & p_Bacteroidetes & c_Bacteroidia & o_Bacteroidales & f_Prevotellaceae & g_Prevotella & $\begin{array}{l}\text { S_Pr } \\
\text { melan }\end{array}$ & $0.00 \%$ & $0.00 \%$ & $0.00 \%$ & $0.00 \%$ \\
\hline k_Bacteria & p_Bacteroidetes & c_Bacteroidia & O_Bacteroidales & f_RF16 & g_Unclassified_RF16 & s_Unclassified_RF16 & $0.00 \%$ & $0.00 \%$ & $0.00 \%$ & $0.01 \%$ \\
\hline Bacteria & p_Bacteroidetes & c_Bacteroidia & O_Bacteroidales & f_Rikenellaceae & & S_indistinctus & $0.00 \%$ & $0.00 \%$ & $0.00 \%$ & $0.02 \%$ \\
\hline Bacteria & p_Bacteroidetes & c_Bacteroidia & o_Bacteroidales & f_Rikenellaceae & $\begin{array}{l}\text { ssified_ } \\
\text { aceae }\end{array}$ & & $0.01 \%$ & $0.00 \%$ & $0.00 \%$ & $0.09 \%$ \\
\hline Bacteria & p_Bacteroidetes & c_Bacteroidia & o_Bacteroidales & f_S24-7 & g_Unclassified_S24-7 & S_Unclassified_S24-7 & $0.19 \%$ & $0.17 \%$ & $0.00 \%$ & $2.53 \%$ \\
\hline
\end{tabular}


Table S3 (continued).

\begin{tabular}{|c|c|c|c|c|c|c|c|c|c|c|}
\hline & & & & & & & Relati & $e a b c$ & danc & f taxa \\
\hline & & & & AUtionity & & & M1 & M2 & M3 & M4 \\
\hline k_Bacteria & p_Bacteroidetes & c_Bacteroidia & O_Bacteroidales & f_SB-1 & g_Unclassified_SB-1 & s_Unclassified_SB-1 & $0.01 \%$ & $0.00 \%$ & $0.00 \%$ & $0.00 \%$ \\
\hline k_Bacteria & p_Bacteroidetes & c_Bacteroidia & O_Bacteroidales & $\begin{array}{l}\text { f_Unclassified_- } \\
\text { Bacteroidales }\end{array}$ & $\begin{array}{l}\text { g_Unclassified_- } \\
\text { Bacteroidales }\end{array}$ & $\begin{array}{l}\text { assified__ } \\
\text { oidales }\end{array}$ & $0.02 \%$ & $0.01 \%$ & $0.00 \%$ & $0.00 \%$ \\
\hline k_Bacteria & p_Bacteroidetes & c_Cytophagia & O_Cytophagales & f_Cytophagaceae & g_Cytophaga & d_Cytophaga & $0.00 \%$ & $0.01 \%$ & $0.00 \%$ & $0.01 \%$ \\
\hline Bacteria & Bacteroidetes & c_Cytophagia & O_Cytophagales & f_Cytophagaceae & $\begin{array}{l}\text { g_Unclassified_ } \\
\text { Cytophagaceae }\end{array}$ & $\begin{array}{l}\text { S_U } \\
\text { cyto }\end{array}$ & $0.00 \%$ & $0.00 \%$ & $0.00 \%$ & $0.00 \%$ \\
\hline k_Bacteria & p_Bacteroidetes & c_Cytophagia & o_Cytophagales & f_Flammeovirgaceae & g_Reichenbachiella & $\begin{array}{l}\text { s.U } \\
\text { Reich }\end{array}$ & $0.00 \%$ & $0.00 \%$ & $0.00 \%$ & $0.00 \%$ \\
\hline k_Bacteria & p_Bacteroidetes & c_Cytophagia & O_Cytophagales & f_Flammeovirgaceae & $\begin{array}{l}\text { g_Unclassified_ } \\
\text { Flammeovirgaceae }\end{array}$ & $\begin{array}{l}\text { s_Unclassified } \\
\text { Flammeovirgaceae }\end{array}$ & $0.09 \%$ & $0.02 \%$ & $0.00 \%$ & $0.63 \%$ \\
\hline k_Bacteria & p_Bacteroidetes & c_Flavobacteriia & o_Flavobacteriales & f_[Weeksellaceae] & g_Chryseobacterium & $\begin{array}{l}\text { s_ } \\
\text { chry }\end{array}$ & $0.03 \%$ & $0.01 \%$ & $0.00 \%$ & $0.01 \%$ \\
\hline k_Bacteria & p_Bacteroidetes & C_Flavobacteriia & O_Flavobacteriales & f_Cryomorphaceae & g_Crocinitomix & $\begin{array}{l}\text { S_Unclassified_- } \\
\text { Crocinitomix }\end{array}$ & $0.00 \%$ & $0.00 \%$ & $0.00 \%$ & $0.02 \%$ \\
\hline k_Bacteria & p_Bacteroidetes & C_Flavobacteriia & O_Flavobacteriales & f_Cryomorphaceae & uviicola & Fluviicola & $0.01 \%$ & $0.01 \%$ & $0.00 \%$ & $0.00 \%$ \\
\hline k_Bacteria & p_Bacteroidetes & C_Flavobacteriia & O_Flavobacteriales & f_Cryomorphaceae & $\begin{array}{l}\text { g_Unclassified_- } \\
\text { Cryomorphaceae }\end{array}$ & $\begin{array}{l}\text { s-o } \\
\text { Cryo } \\
\end{array}$ & $0.00 \%$ & $0.00 \%$ & $0.00 \%$ & $0.01 \%$ \\
\hline k_Bacteria & p_Bacteroidetes & c_Flavobacteriia & o_Flavobacteriales & f_Flavobacteriaceae & g_Capnocytophaga & & $0.00 \%$ & $0.00 \%$ & $0.00 \%$ & $0.00 \%$ \\
\hline k_Bacteria & p_Bacteroidetes & c_Flavobacteriia & o_Flavobacteriales & f_Flavobacteriaceae & g_Capnocytophaga & $\begin{array}{l}\text { ssified_- } \\
\text { ophaga }\end{array}$ & $0.01 \%$ & $0.00 \%$ & $0.00 \%$ & $0.00 \%$ \\
\hline k_Bacteria & p_Bacteroidetes & c_Flavobacteriia & O_Flavobacteriales & f_Flavobacteriaceae & g_Croceimarina & arina_litoralis & $0.01 \%$ & $0.00 \%$ & $0.00 \%$ & $0.00 \%$ \\
\hline k_Bacteria & Bacteroidetes & c_Flavobacteriia & O_Flavobacteriales & f_Flavobacteriaceae & g_Flavobacterium & $\begin{array}{l}\text { sified_- } \\
\text { erium_ }\end{array}$ & $0.00 \%$ & $0.00 \%$ & $0.00 \%$ & $0.00 \%$ \\
\hline k_Bacteria & Bacteroidetes & c_Flavobacteriia & o_Flavobacteriales & f_Flavobacteriaceae & g_Formosa & rassostrea & $0.04 \%$ & $0.00 \%$ & $0.00 \%$ & $0.00 \%$ \\
\hline k_Bacteria & Bacteroidetes & c_Flavobacteriia & o_Flavobacteriales & f_Flavobacteriaceae & g_Lutimonas & Lutimonas & $0.02 \%$ & $0.00 \%$ & $0.00 \%$ & $0.01 \%$ \\
\hline k_Bacteria & p_Bacteroidetes & c_Flavobacteriia & O_Flavobacteriales & f_Flavobacteriaceae & g_Polaribacter & ${ }_{\mathrm{P}}^{\mathrm{S} U}$ & $0.00 \%$ & $0.00 \%$ & $0.00 \%$ & $0.03 \%$ \\
\hline k_Bacteria & p_Bacteroidetes & c_Flavobacteriia & o_Flavobacteriales & f_Flavobacteriaceae & g_Robiginitalea & & $0.03 \%$ & $0.01 \%$ & $0.00 \%$ & $0.00 \%$ \\
\hline k_Bacteria & p_Bacteroidetes & c_Flavobacteriia & o_Flavobacteriales & f_Flavobacteriaceae & $\begin{array}{l}\text { g_Unclassified_ } \\
\text { Flavobacteriaceae }\end{array}$ & sified_ & $0.18 \%$ & $0.06 \%$ & $0.00 \%$ & $0.66 \%$ \\
\hline k_Bacteria & p_Bacteroidetes & C_Sphingobacteriia & $\begin{array}{c}0_{-} \\
\text {Sphingobacteriales }\end{array}$ & f_Sphingobacteriaceae & g_Sphingobacterium & acterium_ & $0.00 \%$ & $0.00 \%$ & $0.00 \%$ & $0.00 \%$ \\
\hline k_Bacteria & p_Bacteroidetes & c_Sphingobacteriia & $\begin{array}{c}\text { O } \\
\text { Sphingobacteriales }\end{array}$ & $\begin{array}{c}\text { f_Unclassified_ } \\
\text { Sphingobacteriales }\end{array}$ & $\begin{array}{r}\text { g_Ur } \\
\text { Sphing }\end{array}$ & $\begin{array}{l}\text { sified_ } \\
\text { teriales }\end{array}$ & $0.00 \%$ & $0.00 \%$ & $0.00 \%$ & $0.01 \%$ \\
\hline k_Bacteria & p_Caldithrix & c_Caldithrixae & O_Caldithrixales & f_Caldithrixaceae & & LCP-26 & $0.01 \%$ & $0.00 \%$ & $0.00 \%$ & $0.00 \%$ \\
\hline Bacteria & p_Chlamydiae & c_Chlamydiia & o_Chlamydiales & $\begin{array}{c}f_{-} \\
\text {Rhabdochlamydiaceae }\end{array}$ & $\begin{array}{r}\text { g_C } \\
\text { Rhabc }\end{array}$ & $\begin{array}{l}\text { _Candi- } \\
\text { hlamydia }\end{array}$ & $0.00 \%$ & $0.00 \%$ & $0.00 \%$ & $0.00 \%$ \\
\hline k_Bacteria & p_Chlorobi & c_BSV26 & O_PK329 & f_Unclassified_PK329 & g_Uncla & d_PK329 & $0.00 \%$ & $0.01 \%$ & $0.00 \%$ & $0.00 \%$ \\
\hline k_Bacteria & p_Chlorobi & Ignavibacteria & o_Ignavibacteriales & f_Ignavibacteriaceae & $\begin{array}{r}\text { g_U } \\
\text { Ignavi }\end{array}$ & $\begin{array}{c}\text { S_Unclassified_ } \\
\text { Ignavibacteriaceae }\end{array}$ & $0.02 \%$ & $0.00 \%$ & $0.00 \%$ & $0.00 \%$ \\
\hline k_Bacteria & p_Chlorobi & c_Ignavibacteria & o_Ignavibacteriales & f_lheB3-7 & $\begin{array}{l}\text { g_Unclassified_heB3- } \\
7\end{array}$ & s_Unclassified_lheB3-7 & $0.01 \%$ & $0.00 \%$ & $0.00 \%$ & $0.01 \%$ \\
\hline k_Bacteria & p_Chloroflexi & c_Anaerolineae & o_Caldilineales & f_Caldilineaceae & & $\begin{array}{l}\text { isified_- } \\
\text { aceae }\end{array}$ & $0.01 \%$ & $0.00 \%$ & $0.00 \%$ & $0.00 \%$ \\
\hline k_Bacteria & p_Chloroflexi & c_Anaerolineae & o_CFB-26 & f_Unclassified_CFB-26 & g_Unclas & s_Unclassified_CFB-26 & $0.01 \%$ & $0.01 \%$ & $0.00 \%$ & $0.00 \%$ \\
\hline k_Bacteria & p_Chloroflexi & c_Anaerolineae & O_GCA004 & f_Unclassified_GCA004 & $\begin{array}{r}\text { g_Unc } \\
\text { Go }\end{array}$ & s_Unclassified_GCA004 & $0.14 \%$ & $0.09 \%$ & $0.00 \%$ & $0.00 \%$ \\
\hline k_Bacteria & p_Chloroflexi & C_Anaerolineae & o_OPB11 & f_Unclassified_OPB11 & $\begin{array}{r}\text { g_Unc } \\
0\end{array}$ & s_Unclassified_OPB11 & $0.01 \%$ & $0.00 \%$ & $0.00 \%$ & $0.00 \%$ \\
\hline k_Bacteria & p_Chloroflexi & C_Anaerolineae & o_S0208 & f_Unclassified_S0208 & g_Unclas & s_Unclassified_S0208 & $0.02 \%$ & $0.01 \%$ & $0.00 \%$ & $0.00 \%$ \\
\hline Bacteria & p_Chloroflexi & Anaerolineae & o_SB-34 & f_Unclassified_s & g_Uncle & ed_SB-34 & $0.00 \%$ & $0.00 \%$ & $0.00 \%$ & $0.00 \%$ \\
\hline Bacteria & p_Chloroflexi & Anae & 1031 & f_A4 & d_A4b & d_A4b & $0.00 \%$ & $0.00 \%$ & $0.00 \%$ & $0.00 \%$ \\
\hline k_Bacteria & p_Chloroflexi & c_Anaerolineae & o_SBR1031 & f_oc28 & d_oc28 & s_Unclassified_oc28 & $0.00 \%$ & $0.00 \%$ & $0.00 \%$ & $0.01 \%$ \\
\hline k_Bacteria & p_Chloroflexi & c_Anaerolineae & o_SBR1031 & f_SHA-31 & g_Unclas & s_Unclassified_SHA-31 & $0.01 \%$ & $0.00 \%$ & $0.00 \%$ & $0.00 \%$ \\
\hline k_Bacteria & p_Chloroflexi & c_Anaerolineae & o_SBR1031 & f_SJA-101 & $\begin{array}{r}\text { g_Unclass } \\
10 \\
\end{array}$ & S_Unclassified_SJA-101 & $0.00 \%$ & $0.00 \%$ & $0.00 \%$ & $0.01 \%$ \\
\hline k_Bacteria & p_Chloroflexi & c_Anaerolineae & O_SHA-20 & f_Unclassified_SHA-20 & 9_Unclass & s_Unclassified_SHA-20 & $0.00 \%$ & $0.01 \%$ & $0.00 \%$ & $0.00 \%$ \\
\hline k_Bacteria & p_Chloroflexi & c_Chloroflexi & o_Chloroflexales & f_FFCH7168 & $\begin{array}{l}\text { g_Ur } \\
\text { FF }\end{array}$ & s_Unclassified_FFCH7168 & $0.00 \%$ & $0.00 \%$ & $0.00 \%$ & $0.01 \%$ \\
\hline k_Bacteria & p_Chloroflexi & $\begin{array}{c}\mathrm{C}_{-} \\
\text {Dehalococcoidete }\end{array}$ & $\begin{array}{l}\text { O_Unclassified_- } \\
\text { Dehalococcoidetes }\end{array}$ & $\begin{array}{c}\text { f_Unclassified_- } \\
\text { Dehalococcoidetes }\end{array}$ & $\begin{array}{c}\text { g_Unclassified } \\
\text { Dehalococcoidetes }\end{array}$ & $\begin{array}{c}\text { S_Unclassified_ } \\
\text { Dehalococcoidetes }\end{array}$ & $0.00 \%$ & $0.00 \%$ & $0.00 \%$ & $0.00 \%$ \\
\hline k_Bacteria & p_Chloroflexi & c_Ellin6529 & & & g_Un & s_Unclassified_Ellin6529 & $0.01 \%$ & $0.01 \%$ & $0.00 \%$ & $0.01 \%$ \\
\hline k_Bacteria & P_Chloroflexi & c_Gitt-GS-136 & $\begin{array}{l}\text { O_Unclassified__ } \\
\text { Gitt-GS-136 }\end{array}$ & f_Unclas & 9_Uncile & s_Unclas & $0.01 \%$ & $0.01 \%$ & $0.00 \%$ & $0.00 \%$ \\
\hline k_Bacteria & p_Chloroflexi & c_Ktedonobacteria & O_JG30-KF-AS9 & $\begin{array}{c}\text { f_Unclassified_JG30- } \\
\text { KF-AS9 }\end{array}$ & $\begin{array}{r}\text { g_Unclas } \\
\mathrm{K} \\
\end{array}$ & eg_JG30- & $0.00 \%$ & $0.00 \%$ & $0.00 \%$ & $0.01 \%$ \\
\hline k_Bacteria & p_Chloroflexi & c_S085 & $\begin{array}{c}\text { O_Unclassified_ } \\
\text { S085 }\end{array}$ & f_Unclassified_S085 & g_Unclassified_S085 & s_Unclassified_S085 & $0.01 \%$ & $0.02 \%$ & $0.00 \%$ & $0.00 \%$ \\
\hline k_Bacteria & p_Chloroflexi & c_Thermomicrobia & o_AKYG1722 & f_Unc & g_Un & s_Unclassified_AKYG1722 & $0.00 \%$ & $0.00 \%$ & $0.00 \%$ & $0.01 \%$ \\
\hline k_Bacteria & p_Chloroflexi & c_Thermomicrobia & o_JG30-KF-CM45 & & $\begin{aligned} \text { g_Uncla } \\
\mathrm{KF}\end{aligned}$ & $\begin{array}{c}\text { s_Unclassified_JG30-KF- } \\
\text { CM45 }\end{array}$ & $0.00 \%$ & $0.00 \%$ & $0.00 \%$ & $0.01 \%$ \\
\hline k_Bacteria & p_Chloroflexi & c_Thermomicrobia & $\begin{array}{c}\mathrm{O}_{-} \\
\text {Thermomicrobiales }\end{array}$ & $\begin{array}{l}\text { f_Uncle } \\
\text { Thermom }\end{array}$ & $\begin{array}{l}\text { g_Un } \\
\text { Therme }\end{array}$ & sified_ & $0.00 \%$ & $0.00 \%$ & $0.00 \%$ & $0.01 \%$ \\
\hline k_Bacteria & p_Chloroflexi & c_TK10 & O_B07_WMSP1 & WMSP1 $^{-}$ & g_Uncla & $\begin{array}{r}\text { s_Uncla } \\
\text { W } \\
\end{array}$ & $0.00 \%$ & $0.00 \%$ & $0.00 \%$ & $0.00 \%$ \\
\hline k_Bacteria & P_Chloroflexi & c_TK17 & o_mle1-48 & f_Unclassified_mle1-48 & $\begin{array}{c}\text { g_Unclassified_mle1- } \\
48\end{array}$ & s_Unclassified_mle1-48 & $0.00 \%$ & $0.00 \%$ & $0.00 \%$ & $0.01 \%$ \\
\hline k_Bacteria & p_Chloroflexi & c_TK17 & $\begin{array}{c}\text { O_Unclassified_ } \\
\text { TK17 }\end{array}$ & f_Unclassified_TK17 & g_Unclassified_TK17 & s_Unclassified_TK17 & $0.00 \%$ & $0.00 \%$ & $0.00 \%$ & $0.00 \%$ \\
\hline k_Bacteria & _Cyanobacteria & c_4COd-2 & o_MLE1-12 & $\begin{array}{c}\text { f_Unclassified_M } \\
12\end{array}$ & $\begin{array}{r}\text { g_Unclassif } \\
12\end{array}$ & s_Unclassified_MLE1-12 & $0.12 \%$ & $0.12 \%$ & $0.00 \%$ & $0.08 \%$ \\
\hline k_Bacteria & p_Cyanobacteria & c_4COd-2 & O_YS2 & f_Unclassifie & g_Unclass & sified_YS2 & $0.00 \%$ & $0.00 \%$ & $0.00 \%$ & $0.06 \%$ \\
\hline k_Bacteria & P_Cyanobacteria & c_Chloroplast & o_Stramenopiles & & & ied_- & $0.03 \%$ & $0.17 \%$ & $0.00 \%$ & $0.02 \%$ \\
\hline k_Bacteria & p_Cyanobacteria & c_Chloroplast & o_Streptophyta & & $\mathrm{SSO}_{\mathrm{Str}}$ & $\begin{array}{l}\text { sified__ } \\
\text { ohyta }\end{array}$ & $1.30 \%$ & $0.22 \%$ & $0.01 \%$ & $0.14 \%$ \\
\hline k_Bacteria & p_Cyanobacteria & c_ML635J-21 & $\begin{array}{c}\text { O_Unclassified_ } \\
\text { ML635J-21 }\end{array}$ & $\begin{array}{l}\text { fUUnclassified } \\
\text { ML635J-21 }\end{array}$ & $\begin{array}{l}\text { g_Uncl } \\
\text { ML63 }\end{array}$ & s_Unclass & $0.00 \%$ & $0.00 \%$ & $0.00 \%$ & $0.00 \%$ \\
\hline k_Bacteria & p_Cyanobacteria & $\begin{array}{c}\text { C_Synecho- } \\
\text { coccophycideae }\end{array}$ & $\begin{array}{c}0_{-} \\
\text {Synechococcales }\end{array}$ & f_Synechococcaceae & g_Synechococcus & & $0.00 \%$ & $0.01 \%$ & $0.00 \%$ & $0.00 \%$ \\
\hline k_Bacteria & p_Elusimicrobia & c_Elusimicrobia & O_Elusimicrobiales & $\begin{array}{l}\text { f_Unclassified_ } \\
\text { Elusimicrobiales }\end{array}$ & $\begin{array}{l}\text { g_Uncla } \\
\text { Elusimic }\end{array}$ & & $0.01 \%$ & $0.01 \%$ & $0.00 \%$ & $0.01 \%$ \\
\hline k_Bacteria & p_Firmicutes & c_Bacilli & o_Bacillales & f_[Exiguobacteraceae] & g_Exiguobacterium & & $0.01 \%$ & $0.14 \%$ & $0.02 \%$ & $0.00 \%$ \\
\hline Bacteria & p_Firmicutes & c_Bacilli & o_Bacillales & f_[Exiguobacteraceae] & $\begin{array}{c}\text { g_Unclassified_ } \\
\text { [Exiguobacteraceae] }\end{array}$ & SExigi & $0.00 \%$ & $0.00 \%$ & $0.00 \%$ & $0.00 \%$ \\
\hline k_Bacteria & p_Firmicutes & c_Bacilli & o_Bacillales & f_Bacillaceae & g_Anoxybacillus & s.An & $0.02 \%$ & $0.01 \%$ & $0.00 \%$ & $0.02 \%$ \\
\hline
\end{tabular}


Table S3 (continued).

Taxonomy

k_Bacteria p_Firmicutes c_Bacilli o_Bacillales

k_Bacteria $\quad$ p_Firmicutes c_Bacilli $\quad$ o_Bacillales

k_Bacteria p_Firmicutes c_Bacilli o_Bacillales

k_Bacteria p_Firmicutes c_Bacilli o_Bacillales

k_Bacteria p_Firmicutes c_Bacilli o_Bacillales

k_Bacteria p_Firmicutes c_Bacilli o_Bacillales

k_Bacteria p_Firmicutes c_Bacilli o_Bacillales

k_Bacteria p_Firmicutes c_Bacilli $\quad$ o_Bacillales

k_Bacteria p_Firmicutes c_Bacilli o_Bacillales

k_Bacteria p_Firmicutes c_Bacilli o_Bacillales

k_Bacteria p_Firmicutes c_Bacilli o_Bacillales

k_Bacteria p_Firmicutes c_Bacilli o_Bacillales

k_Bacteria p_Firmicutes c_Bacilli o_Gemellales

k_Bacteria p_Firmicutes c_Bacilli o_Lactobacillales

k_Bacteria $\quad$ p_Firmicutes $\quad$ c_Bacilli $\quad$ o_Lactobacillales

k_Bacteria p_Firmicutes c_Bacilli o_Lactobacillales

k_Bacteria p_Firmicutes c_Bacilli o_Lactobacillales

k_Bacteria p_Firmicutes c_Bacilli o_Lactobacillales

k_Bacteria p_Firmicutes c_Bacilli o_Lactobacillales

k_Bacteria p_Firmicutes c_Bacilli $\quad$ o_Lactobacillales

k_Bacteria p_Firmicutes c_Bacilli o_Lactobacillales

k_Bacteria p_Firmicutes c_Bacilli o_Lactobacillales

k_Bacteria $\quad$ p_Firmicutes c_Bacilli o_Lactobacillales

k_Bacteria p_Firmicutes c_Bacilli o_Lactobacillales

k_Bacteria $\quad$ p_Firmicutes c_Bacilli $\quad$ o_Lactobacillales

k_Bacteria p_Firmicutes c_Bacilli o_Lactobacillales

k_Bacteria p_Firmicutes c_Bacilli o_Lactobacillales

k_Bacteria p_Firmicutes c_Bacilli o_Lactobacillales

k_Bacteria p_Firmicutes c_Bacilli o_Lactobacillales

k_Bacteria p_Firmicutes c_Bacilli o_Lactobacillales

k_Bacteria p_Firmicutes c_Bacilli o_Lactobacillales

k_Bacteria p_Firmicutes c_Bacilli o_Lactobacillales

k_Bacteria p_Firmicutes c_Bacilli o_Lactobacillales

k_Bacteria p_Firmicutes c_Bacilli o_Turicibacterales

k_Bacteria P_Firmicutes c_Clostridia o_Clostridiales

k_Bacteria p_Firmicutes c_Clostridia o_Clostridiales

k_Bacteria p_Firmicutes c_Clostridia o_Clostridiales

k_Bacteria P_Firmicutes C_Clostridia o_Clostridiales

k_Bacteria p_Firmicutes c_Clostridia o_Clostridiales

k_Bacteria p_Firmicutes C_Clostridia o_Clostridiales

k_Bacteria p_Firmicutes c_Clostridia o_Clostridiales

k_Bacteria p_Firmicutes C_Clostridia o_Clostridiales

k_Bacteria p_Firmicutes c_Clostridia o_Clostridiales

k_Bacteria p_Firmicutes c_Clostridia o_Clostridiales

k_Bacteria p_Firmicutes c_Clostridia o_Clostridiales

k_Bacteria p_Firmicutes c_Clostridia o_Clostridiales

k_Bacteria p_Firmicutes c_Clostridia o_Clostridiales

k_Bacteria p_Firmicutes C_Clostridia o_Clostridiales

k Bacteria p Firmicutes c Clostridia o Clostridiales

k_Bacteria P_Firmicutes c_Clostridia o_Clostridiales

k_Bacteria p_Firmicutes c_Clostridia o_Clostridiales

k_Bacteria p_Firmicutes c_Clostridia o_Clostridiales

k_Bacteria p_Firmicutes c_Clostridia o_Clostridiales

k_Bacteria p_Firmicutes C_Clostridia o_Clostridiales

k_Bacteria p_Firmicutes c_Clostridia o_Clostridiales

k_Bacteria p_Firmicutes c_Clostridia o_Clostridiales

k_Bacteria p_Firmicutes c_Clostridia o_Clostridiales

k_Bacteria p_Firmicutes c_Clostridia o_Clostridiales

k_Bacteria p_Firmicutes c_Clostridia o_Clostridiales

k_Bacteria p_Firmicutes c_Clostridia o_Clostridiales

k_Bacteria p_Firmicutes c_Clostridia o_Clostridiales

k_Bacteria P_Firmicutes c_Clostridia o_Clostridiales

k_Bacteria p_Firmicutes c_Clostridia o_Clostridiales

k_Bacteria p_Firmicutes c_Clostridia o_Clostridiales

k_Bacteria p_Firmicutes C_Clostridia o_Clostridiales

k_Bacteria p_Firmicutes C_Clostridia o_Clostridiales

k_Bacteria p_Firmicutes c_Clostridia o_Clostridiales

k_Bacteria p_Firmicutes c_Clostridia o_Clostridiales

k_Bacteria p_Firmicutes c_Clostridia o_Clostridiales f Bacillaceae

f_Bacillaceae

f_Listeriaceae

f_Paenibacillaceae

f_Planococcaceae

f_Planococcaceae

f_Planococcaceae

f_Staphylococcaceae

f_Staphylococcaceae

Staphylococcaceae

f_Staphylococcaceae

f_Thermoactino-

mycetaceae

Gemellaceae

f_Aerococcaceae

f_Aerococcaceae

f_Aerococcaceae

f_Carnobacteriaceae

f_Carnobacteriaceae

f_Enterococcaceae

f_Enterococcaceae

f_Enterococcaceae

f Lactobacillaceae

f_Lactobacillaceae

f_Lactobacillaceae

f_Lactobacillaceae

f_Lactobacillaceae

f_Leuconostocaceae

f_Leuconostocaceae

f_Streptococcaceae

f_Streptococcaceae

f_Streptococcaceae

f_Streptococcaceae

f_Unclassified_

f_Turicibacteraceae

f_[Mogibacteriaceae] f_XIII

f_[Mogibacteriaceae]

f_[Tissierellaceae]

f_[Tissierellaceae]

Caldicoprobacteraceae

f_Christensenellaceae

f_Clostridiaceae

f_Clostridiaceae

f_Clostridiaceae

f_Eubacteriaceae

f Lachnospiraceae

f_Lachnospiraceae

f_Lachnospiraceae

f Lachnospiraceae

f_Lachnospiraceae

f_Lachnospiraceae

f_Lachnospiraceae

f_Lachnospiraceae

f_Lachnospiraceae

f_Lachnospiraceae

f_Lachnospiraceae

f_Lachnospiraceae

f_Peptococcaceae

f_Peptococcaceae

f_Peptococcaceae

f Peptostreptococcaceae

f_Peptostreptococcaceae

f_Peptostreptococcaceae

Peptostreptococcaceae

f_Ruminococcaceae

f_Ruminococcaceae

f_Ruminococcaceae

f_Ruminococcaceae

f_Ruminococcaceae
Relative aboundance of taxa \begin{tabular}{cccc} 
M1 & M2 & M3 & M4 \\
\hline $0.00 \%$ & $0.01 \%$ & $0.02 \%$ & $0.00 \%$ \\
\hline $0.09 \%$ & $0.08 \%$ & $0.00 \%$ & $0.15 \%$
\end{tabular} \begin{tabular}{cccccc} 
g_Bacillus & S_Unclassified_Bacillus & $0.00 \%$ & $0.01 \%$ & $0.02 \%$ & $0.01 \%$ \\
g_Geobacillus & s_Unclassified_Geobacillus & $0.09 \%$ & $0.08 \%$ & $0.00 \%$ & $0.15 \%$ \\
\hline
\end{tabular} \begin{tabular}{llllll} 
g_Listeria & S_Listeria_seeligeri & $0.00 \%$ & $0.00 \%$ & $0.18 \%$ & $0.01 \%$ \\
\hline
\end{tabular} $\begin{array}{llllll}\text { g_Unclassified__ } & \text { s_Unclassified_Paenibacillaceae } & 0.00 \% & 0.00 \% & 0.00 \% & 0.00 \%\end{array}$ $\begin{array}{llllll}\text { g_Rummelibacillus } & \text { s_Unclassified_Rummeliibacillus } & 0.00 \% & 0.00 \% & 0.00 \% & 0.00 \%\end{array}$ \begin{tabular}{llllll} 
g_Solibacillus & s_Unclassified_Solibacillus & $0.03 \%$ & $0.00 \%$ & $0.00 \%$ & $0.00 \%$ \\
\hline
\end{tabular} $\begin{array}{lllllll}\text { g_Unclassified_- } & \text { s_Unclassified_Planococcaceae } & 0.01 \% & 0.03 \% & 0.30 \% & 0.01 \%\end{array}$ \begin{tabular}{llllll} 
g_Jeotgalicoccus & s_Jeotgalicoccus_psychrophilus & $0.00 \%$ & $0.00 \%$ & $0.00 \%$ & $0.02 \%$ \\
\hline
\end{tabular} $\begin{array}{llllll}\text { g_Staphylococcus } & \text { s_Staphylococcus_epidermidis } & 0.06 \% & 0.10 \% & 0.00 \% & 0.08 \%\end{array}$ \begin{tabular}{lllllll}
\hline g_Staphylococcus & s Staphylococcus_sciuri & $0.06 \%$ & $0.10 \%$ & $0.00 \%$ & $0.03 \%$ \\
\hline
\end{tabular} \begin{tabular}{llllll} 
g_Staphylococcus & s_Unclassified_Staphylococcus & $0.00 \%$ & $0.00 \%$ & $0.02 \%$ & $0.00 \%$ \\
\hline
\end{tabular}

g_Thermoactinomyces Thermoactinomyces g_Unclassified g_Aerococcus \begin{tabular}{lllllll} 
g_Alloiococcus & S_Unclassified_Aerococcus & $0.00 \%$ & $0.00 \%$ & $0.00 \%$ & $0.00 \%$ \\
\hline
\end{tabular} \begin{tabular}{llllll} 
g_Unclassified_Alloiococcus & $0.00 \%$ & $0.00 \%$ & $0.00 \%$ & $0.01 \%$ \\
\hline
\end{tabular} \begin{tabular}{llllll} 
Aerococcaceae & s_Unclassified_Aerococcaceae & $0.01 \%$ & $0.01 \%$ & $0.00 \%$ & $0.00 \%$ \\
\hline
\end{tabular} \begin{tabular}{llllll} 
g_Granulicatella & s_Unclassified_Granulicatella & $0.10 \%$ & $0.02 \%$ & $0.00 \%$ & $0.00 \%$ \\
\hline
\end{tabular} \begin{tabular}{llllll} 
g_Isobaculum & s_Isobaculum_melis & $0.00 \%$ & $0.01 \%$ & $0.00 \%$ & $0.00 \%$ \\
\hline
\end{tabular} $\begin{array}{llllll}\text { g_Enterococcus } & \text { s_Enterococcus_cecorum } & 0.04 \% & 0.00 \% & 0.00 \% & 0.00 \%\end{array}$ \begin{tabular}{llllll} 
g_Enterococcus & S_Unclassified_Enterococcus & $26.51 \%$ & $4.75 \%$ & $5.93 \%$ & $2.59 \%$ \\
\hline
\end{tabular} \begin{tabular}{llllll} 
g_Vagococcus & s_Unclassified_Vagococcus & $0.01 \%$ & $0.00 \%$ & $0.00 \%$ & $0.00 \%$ \\
\hline
\end{tabular} $\begin{array}{llllll}\text { g_Lactobacillus } & \text { s_Lactobacillus_brevis } & 0.00 \% & 0.00 \% & 0.02 \% & 0.00 \%\end{array}$ \begin{tabular}{llllll} 
g_L_actobacillus & s_Lactobacillus_iners & $0.00 \%$ & $0.01 \%$ & $0.00 \%$ & $0.01 \%$ \\
\hline
\end{tabular} \begin{tabular}{llllll} 
g_Lactobacillus & s_Lactobacillus_zeae & $0.00 \%$ & $0.00 \%$ & $0.00 \%$ & $0.00 \%$ \\
\hline
\end{tabular} \begin{tabular}{llllll} 
g_Lactobacillus & s_Unclassified_Lactobacillus & $0.55 \%$ & $0.11 \%$ & $0.00 \%$ & $0.24 \%$ \\
\hline
\end{tabular} \begin{tabular}{llllll} 
g_Unclassified__ & s_Unclassified_Lactobacillaceae & $0.02 \%$ & $0.02 \%$ & $0.01 \%$ & $0.05 \%$ \\
\hline
\end{tabular} \begin{tabular}{llllll} 
L_Lactobacillaceas & s_Unclassified_Leuconostoc & $0.00 \%$ & $0.00 \%$ & $0.00 \%$ & $0.00 \%$ \\
\hline
\end{tabular} $\begin{array}{llllll}\text { g_Unclassified____Unclassified_Leuconostocaceae } & 0.00 \% & 0.00 \% & 0.00 \% & 0.01 \%\end{array}$ \begin{tabular}{cccccc} 
g_Lactococcus & s_Lactococcus_garvieae & $0.00 \%$ & $0.00 \%$ & $0.01 \%$ & $0.00 \%$ \\
\hline
\end{tabular} \begin{tabular}{llllll} 
g_Lactococcus & s_Unclassified_Lactococcus & $0.01 \%$ & $0.01 \%$ & $0.10 \%$ & $0.01 \%$ \\
\hline
\end{tabular} \begin{tabular}{llllll} 
g_Streptococcus & s_Unclassified_Streptococcus & $2.32 \%$ & $0.33 \%$ & $0.00 \%$ & $0.03 \%$ \\
\hline
\end{tabular} $\begin{array}{llllll}\text { g_Unclassified__ } & \text { s_Unclassified_Streptococcaceae } & 0.03 \% & 0.01 \% & 0.04 \% & 0.00 \%\end{array}$ \begin{tabular}{cccccc} 
Streptococcaceae & s_Unclassified_Streptococcaceae & $0.03 \%$ & $0.01 \%$ & $0.04 \%$ & $0.00 \%$ \\
\hline g_Unclassified_- & s_Unclassified_Lactobacillales & $0.28 \%$ & $0.09 \%$ & $0.29 \%$ & $0.05 \%$
\end{tabular} \begin{tabular}{llllll} 
Lactobacillales & s_Unclassified_Lactobacillales & $0.28 \%$ & $0.09 \%$ & $0.29 \%$ & $0.05 \%$ \\
\hline g_Turicibacter & s_Unclassified_Turicibacter & $0.02 \%$ & $0.00 \%$ & $0.00 \%$ & $0.00 \%$ \\
\hline
\end{tabular} \begin{tabular}{llllll} 
g_Anaerovorax & S_Unclassified_Anaerovorax & $0.01 \%$ & $0.00 \%$ & $0.00 \%$ & $0.00 \%$ \\
\hline
\end{tabular} \begin{tabular}{cccccc} 
g_Anaerovorax & S_Unclassified_Anaerovorax & $0.01 \%$ & $0.00 \%$ & $0.00 \%$ & $0.00 \%$ \\
\hline [Eubacterium] nodatum & S_Unclassified_[Eubacterium] & $0.00 \%$ & $0.00 \%$ & $0.00 \%$ & $1.75 \%$
\end{tabular} g_unclassified

\begin{tabular}{llllll} 
[Mogibacteriaceae] & $0.04 \%$ & $0.00 \%$ & $0.00 \%$ & $1.87 \%$ \\
\hline
\end{tabular} $\begin{array}{llllll}\text { g_Anaerococcus } & \text { s_Unclassified_Anaerococcus } & 0.00 \% & 0.00 \% & 0.00 \% & 0.03 \%\end{array}$ \begin{tabular}{llllll} 
g_Parvimonas & s_Unclassified_Parvimonas & $0.01 \%$ & $0.01 \%$ & $0.00 \%$ & $0.00 \%$ \\
\hline
\end{tabular} \begin{tabular}{llllll} 
g_Caldicoprobacter & s_Unclassified_Caldicoprobacter & $0.01 \%$ & $0.01 \%$ & $0.00 \%$ & $0.01 \%$ \\
\hline
\end{tabular} g_Unclassified_ s_Unclassified \begin{tabular}{llllll} 
g_Clostridium & S Christensenellaceae & $0.00 \%$ & $0.00 \%$ & $0.00 \%$ & $0.02 \%$ \\
\hline
\end{tabular} \begin{tabular}{llllll} 
g_Clostridium & s_Unclassified_Clostridium & $0.02 \%$ & $0.04 \%$ & $0.09 \%$ & $0.06 \%$ \\
\hline
\end{tabular} $\begin{array}{llllll}\text { g_Unclassified_- } & \text { s_Unclassified_Clostridiaceae } & 0.09 \% & 0.02 \% & 0.00 \% & 0.50 \%\end{array}$ g_Pseudroramibacter_ _ s_Unclassified_

\begin{tabular}{llllll} 
Eubacterium - Pseudoramibacter_Eubacterium & $0.01 \%$ & $0.01 \%$ & $0.03 \%$ & $0.00 \%$ \\
\hline
\end{tabular} \begin{tabular}{llllll} 
& $0.00 \%$ \\
\hline
\end{tabular} \begin{tabular}{llllll} 
g_[Ruminococcus] & s_Unclassified_[Ruminococcus] & $0.00 \%$ & $0.00 \%$ & $0.00 \%$ & $0.00 \%$ \\
\hline
\end{tabular} \begin{tabular}{llllll} 
g_Blautia & s_Blautia_producta & $0.00 \%$ & $0.00 \%$ & $0.00 \%$ & $0.01 \%$ \\
\hline
\end{tabular} \begin{tabular}{llllll} 
g_Blautia & s_Unclassified_Blautia & $0.01 \%$ & $0.01 \%$ & $0.00 \%$ & $0.02 \%$ \\
\hline
\end{tabular} g_Catonella \begin{tabular}{cccccc} 
g_Coprococcus & s_Unclassified_Coprococcus & $0.00 \%$ & $0.01 \%$ & $0.00 \%$ & $0.02 \%$ \\
\hline g Dorea & s Unclassified Dorea & $0.01 \%$ & $0.03 \%$ & $0.00 \%$ & $0.27 \%$ \\
\hline
\end{tabular}

s Unclassified Caton \begin{tabular}{llll}
$0.01 \%$ & $0.00 \%$ & $0.00 \%$ & $0.00 \%$ \\
\hline
\end{tabular} \begin{tabular}{llllll} 
g_Epulopiscium & s_Unclassified_Epulopiscium & $0.00 \%$ & $0.00 \%$ & $0.00 \%$ & $0.01 \%$ \\
\hline
\end{tabular} \begin{tabular}{llllll} 
g_Moryella & s_Unclassified_Moryella & $0.04 \%$ & $0.00 \%$ & $0.00 \%$ & $0.00 \%$ \\
\hline
\end{tabular} \begin{tabular}{llllll} 
g_Oribacterium & s_Unclassified_Oribacterium & $0.23 \%$ & $0.03 \%$ & $0.00 \%$ & $0.00 \%$ \\
\hline
\end{tabular} g Roseburia g-Unclassified

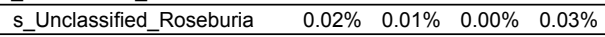
$\begin{array}{lllllll}\text { g_Unclassified_- } & \text { s_Unclassified_Lachnospiraceae } & 0.11 \% & 0.39 \% & 0.64 \% & 0.17 \%\end{array}$ $\begin{array}{llllll}\begin{array}{c}\text { Desulfotomaculum_ } \\ \text { Desulfovirgula }\end{array} & \begin{array}{c}\text { S_Unclassified_- } \\ \text { Desulfotomaculum Desulfovirgula }\end{array} & 0.00 \% & 0.00 \% & 0.00 \% & 0.00 \%\end{array}$ \begin{tabular}{cccccc} 
g_rc4-4 & s_Unclassified_rc4-4 & $0.00 \%$ & $0.00 \%$ & $0.00 \%$ & $0.06 \%$ \\
\hline
\end{tabular} $\begin{array}{llllll}\text { g_Unclassified__ } & \text { S_Unclassified_Peptococcaceae } & 0.00 \% & 0.00 \% & 0.00 \% & 0.09 \%\end{array}$ \begin{tabular}{llllll} 
g_Clostridium & s_Clostridium_venationis & $0.00 \%$ & $0.02 \%$ & $0.38 \%$ & $0.01 \%$ \\
\hline
\end{tabular} \begin{tabular}{llllll} 
g_Filifactor & s_Unclassified_Filifactor & $0.01 \%$ & $0.00 \%$ & $0.00 \%$ & $0.00 \%$ \\
\hline
\end{tabular} $\begin{array}{cccccc}\text { g_Tepidibacter } & \text { s_Unclassified_Tepidibacter } & 0.01 \% & 0.00 \% & 0.00 \% & 0.00 \% \\ \text { g_Unclassified_ } & \text { S_Unclassified_ } & 0.15 \% & 2.89 \% & 3.55 \% & 0.28 \%\end{array}$

\begin{tabular}{lccccc}
\hline g Faecalibacterium & P Feptostreptococcaceae & $0.15 \%$ & $2.89 \%$ & $3.55 \%$ & $0.28 \%$ \\
\hline F Faecalibacterium prausnitzii & $0.00 \%$ & $0.01 \%$ & $0.00 \%$ & $0.01 \%$ \\
\hline
\end{tabular} g_Ruminococcus \begin{tabular}{llllll} 
g_Ruminococcus & s_Unclassified_Ruminococcus & $0.02 \%$ & $0.01 \%$ & $0.00 \%$ & $0.16 \%$ \\
\hline
\end{tabular} g_Unclassified
Ruminococcace 
Table S3 (continued)

\begin{tabular}{|c|c|c|c|c|c|c|c|c|c|}
\hline & & & & & & & Relative abou & dance & e of taxa \\
\hline & & & Un & & & & $\begin{array}{ll}\text { M1 } & \text { M2 } \\
\end{array}$ & M3 & $\mathrm{M} 4$ \\
\hline Bacteria & Firmicutes & Clostridia & Clostridiales & f_Veillonellaceae & g_Dialister & s_Unclassified_Dialister & $0.00 \% \quad 0.00 \%$ & $0.00 \%$ & $.00 \%$ \\
\hline Bacteria & p_Firmicutes & c_Clostridia & o_Clostridiales & f_Veillonellaceae & & $\begin{array}{l}\text { s_Unclassified_- } \\
\text { Phascolarctobacterium }\end{array}$ & $0.00 \% \quad 0.00 \%$ & $6.00 \%$ & $\% 0.01 \%$ \\
\hline Bacteria & Firmicutes & c_Clostridia & o_Clostridiales & Veillonellaceae & g_Schwartzia & issified_ & $0.03 \% 0.00 \%$ & $6.00 \%$ & $\% 0.00 \%$ \\
\hline Bacteria & Firmicutes & Clostridia & O_Clostridiales & Veillonellaceae & Selenomonas & noxia & $0.01 \% \quad 0.01 \%$ & $0.00 \%$ & 0.00 \\
\hline E_Bacteria & p_Firmicutes & c_Clostridia & o_Clostridiales & f_Veillonellaceae & Selenomonas & & $0.02 \% 0.00 \%$ & $6.00 \%$ & $\% 0.00$ \\
\hline k_Bacteria & p_Firmicutes & c_Clostridia & o_Clostridiales & f_Veillonellaceae & $\begin{array}{l}\text { Unclassified_- } \\
\text { jillonellaceae }\end{array}$ & $\begin{array}{ll}\text { s. } \\
\text { Veil }\end{array}$ & $0.00 \% \quad 0.00 \%$ & $6.00 \%$ & $\% 0.04 \%$ \\
\hline Bacteria & p_Firmicutes & c_Clostridia & o_Clostridiales & f_Veillonellaceae & g_Veillonella & & $0.01 \% \quad 0.00 \%$ & $6.00 \%$ & $\% 0.00 \%$ \\
\hline Bacteria & p_Firmicutes & Clostridia & o_Clostridiales & eae & g_Veillonella & dispar & $0.02 \% 0.01 \%$ & $0.00 \%$ & $0.00^{\circ}$ \\
\hline Bacteria & Firn & Clo & o_Clostrid & & g_Veillor & s_Veilld & $0.10 \% \quad 0.02 \%$ & $0.00 \%$ & $0.00^{\circ}$ \\
\hline k_Bacteria & p_Firmicutes & c_Clostridia & $\begin{array}{c}0_{-} \\
\text {moanaerob }\end{array}$ & $\begin{array}{l}\text { for } \\
\text { ermoar }\end{array}$ & $\begin{array}{l}\text { g_Unclassified_- } \\
\text { ermoanaerobactera }\end{array}$ & $\begin{array}{l}\text { Ssified_- } \\
\text { obacterales }\end{array}$ & $0.00 \% \quad 0.00 \%$ & $6.00 \%$ & $0.00 \%$ \\
\hline Bacteria & P_Firmicutes & Erysipelotrichi & Erysipelotrichales & f_Erysipelotrichaceae & g_[Eubacterium] & & $0.00 \% \quad 0.01 \%$ & $6.00 \%$ & $\% 0.00 \%$ \\
\hline Bacteria & p_Firmicutes & c_Erysipelotrichi & o_Erysipelotrichales & f_Erysipelotrichaceae & g_Allobaculum & & $0.06 \% 0.06 \%$ & $6.00 \%$ & $\% 2.12 \%$ \\
\hline Bacteria & p_Firmicutes & Erysipelotrichi & Erysipelotrichales & Erysipelotrichaceae & $\mathrm{g}_{-} \mathrm{Bu}$ & & $0.05 \% 0.00 \%$ & $0.00 \%$ & $0.00 \%$ \\
\hline k_Bacteria & p_Firmicutes & c_Erysipelotrichi & o_Erysipelotrichales & f_Erysipelotrichaceae & g_Ur & & $0.00 \% 0.00 \%$ & $0.02 \%$ & $\% 0.01 \%$ \\
\hline __Bacteria & p_Fusobacteria & c_Fusobacteriia & O_Fusobacteriales & f_Fusobacteriaceae & g_Cetobacterium & & $0.13 \% \quad 0.04 \%$ & $6.00 \%$ & $\% 0.00$ \\
\hline ¿_Bacteria & p_Fusobacteria & c_Fusobacteriia & o_Fusobacteriales & f_Fusobacteriaceae & g_Fusobacterium & & $0.05 \% \quad 0.01 \%$ & $6.00 \%$ & $\% 1.87 \%$ \\
\hline __Bacteria & P_Fusobacteria & c_Fusobacteriia & O_Fusobacteriales & f_Fusobacteriaceae & g_Propionigenium & & $0.00 \% 0.01 \%$ & $60.00 \%$ & $\% 0.00^{\circ}-2$ \\
\hline ¿_Bacteria & p_Fusobacteria & c_Fusobacteriia & O_Fusobacteriales & f_Leptotrichiaceae & g_Leptotrichia & & $0.12 \% \quad 0.02 \%$ & $6.00 \%$ & $\% 0.00 \%$ \\
\hline k_Bacteria & $\begin{array}{c}p_{-} \\
\text {emmatimonadetes }\end{array}$ & C_Gemm-5 & _Unclassified_Gemm-5 & 5 f_Unclassified_Gemm & g_Unclassified_Gemm- & s_Unclassified_Gemm-5 & $0.09 \% \quad 0.04 \%$ & $6.00 \%$ & $\% 0.019$ \\
\hline k_Bacteria & emmatimona & $\begin{array}{c}\mathrm{C}_{-} \\
\text {nmatimonade }\end{array}$ & O_C114 & f_Unclassified_C114 & g_Unclassified_C114 & s_Unclassified_C114 & $0.00 \% \quad 0.00 \%$ & 0.00 & $\% 0.01$ \\
\hline k_Bacteria & $\begin{array}{l}\frac{p_{1}}{p_{-}} \\
\text {patimonade }\end{array}$ & $\begin{array}{l}\text { nmattimonac } \\
\text { nmatimonac } \\
\text { nacion }\end{array}$ & O_Ellin5290 & f_Unclassified_Ellin5290 & $\begin{array}{l}\text { g_Unclassified_- } \\
\text { Ellin5290 }\end{array}$ & $\begin{array}{c}\text { s_Unclassified__ } \\
\text { Ellin5290 }\end{array}$ & $0.00 \% \quad 0.01 \%$ & $6.00 \%$ & $0.00 \%$ \\
\hline Bacteria & $\begin{array}{l}p_{-} \\
\text {emmatimonade }\end{array}$ & $\begin{array}{l}C_{-} \\
\text {mmatimonad }\end{array}$ & o_KD8-87 & _Unclassified_KD8-87 & g_Unclassified_KD8-87 & s_Unclassified_KD8-87 & $0.01 \% \quad 0.00 \%$ & & \\
\hline k_Bacteria & $\begin{array}{c}\mathrm{p}_{-} \\
\text {Semmatimonade }\end{array}$ & $\frac{c_{-}}{c_{-}}$ & $\begin{array}{l}\text { O_Unclassified }_{-} \\
\text {Gemmatimonadetes }\end{array}$ & f_Unclassified_- & & & $0.00 \% \quad 0.00 \%$ & $6.00 \%$ & $\% 0.00 \%$ \\
\hline Bacteria & p GNO2 & & & & & & $0.01 \% \quad 0.00 \%$ & $0.00 \%$ & 0.019 \\
\hline cte & P_GN & & & Un & & & & & \\
\hline cteria & P_GN04 & & S & f_Uncle & g_Unclassified_GN15 & ified_GN15 & $0.00 \% \quad 0.00 \%$ & $0.00 \%$ & $0.00 \%$ \\
\hline k_Bacteria & p_KSB3 & 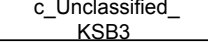 & o_Unclassified_KSB3 & f_Unclassified_KSB3 & g_Unclassified_KSB3 & s_Unclassified_KSB3 & $0.00 \% 0.00 \%$ & $6.00 \%$ & $\% 0.00^{\circ}$ \\
\hline k_Bacteria & p_Nitrospirae & c_Nitrospira & o_Nitrospirales & $f-1 T$ & g_BD2-6 & s_Unclassified_BD2-6 & $0.07 \% \quad 0.02 \%$ & $6.00 \%$ & $\% 0.00 \%$ \\
\hline Bacteria & p_Nitrospirae & c_Nitrospira & o_Nitrospirales & desultovI & g_GOUTA19 & $\begin{array}{l}\text { Ssified_ } \\
\text { AA19 }\end{array}$ & $0.03 \% 0.03 \%$ & $6.00 \%$ & $\% 0.00$ \\
\hline Bacteria & p_Nitrospirae & c_Nitrospira & o_Nitrospirales & $\begin{array}{c}\text { fIT } \\
\text { desulfovi }\end{array}$ & g_Unclassified_Ther & & $0.01 \% \quad 0.00 \%$ & $6.00 \%$ & $\% 0.00 \%$ \\
\hline Bacteria & Nitrospirae & C_Nitrospira & o_Nitrospirales & f_0319-6A21 & & & $0.00 \% 0$. & & $\% 0.00$ \\
\hline K_Bacteria & P_Nitrospirae & c_Nitrospira & o_Nitrospirales & f_Nitrospiraceae & & & $0.02 \% \quad 0.02 \%$ & $6.00 \%$ & $\% 0.01^{\circ}$ \\
\hline Bacteria & p OD1 & c_ABY1 & Unclas & $3 Y 1$ & & BY1 & & & $\% 0.00$ \\
\hline Bacteria & P_C & & & & & & $\% 0$ & & $\% 0.01$ \\
\hline k_Bacteria & p_OP1 & c_[Acetothermia] & o_[Acetothermales] & & & & $0.00 \% 0.01 \%$ & & $\% 0.00^{\circ}>-3$ \\
\hline k_Bacteria & P_OP8 & C_OP8_1 & O_HMMVPog-54 & & & & $0.00 \% 0.0$ & 60.00 & $\% 0.00^{\circ}$ \\
\hline k_Bacteria & Planctomycetes & C_C6 & o_d113 & & & & $0.01 \% \quad 0.00 \%$ & $0.00 \%$ & $0.00 \%$ \\
\hline k_Bacteria & Planctomycetes & c_OM190 & o_CL500-15 & & & & $0.01 \% \quad 0.01$ & 0.0 & $\% 0.00$ \\
\hline E_Bacteria & Planctomycetes & C_Phycisphaerae & Phycisphaerales & f_Phycisphaeraceae & & & $0.00 \% \quad 0.01 \%$ & $6.00 \%$ & $\% 0.00 \%$ \\
\hline Bacteria & Planctomycetes & c_Phycisphaerae & erales & & & & $0.05 \% 0.0$ & & $0.03 \%$ \\
\hline EBacteria & Planctomycetes & C_Pla3 & O_Unclassified_Pla3 & f_Unclassified_Pla3 & & & $0.00 \% \quad 0.00 \%$ & $0.00 \%$ & $0.00 \%$ \\
\hline k_Bacteria & p_Planctomycetes & c_Planctomycetia & o_Gemmatales & f_Isosphaeraceae & & & $0.01 \% \quad 0.00 \%$ & $6.00 \%$ & $0.02 \%$ \\
\hline k_Bacteria & p_Proteobacteria & & O_BD7-3 & Unclassified_BD7-3 & Unclassified_BD7-3 & BD7-3 & $0.00 \% \quad 0.00 \%$ & $6.00 \%$ & $\% 0.01$ \\
\hline Bacteria & p_Proteobacteria & & o_Caulobacterales & f_Caulobacteraceae & g_Arthrospira & ied & $0.00 \% \quad 0.00 \%$ & $6.00 \%$ & $\% 0.00$ \\
\hline Bacteria & Proteobacteria & oleop & O_Caulobacterales & f_Caulobacteraceae & g_Asticcacaulis & & $0.05 \% 0.05$ & & \\
\hline K_Bacteria & p_Proteobacteria & fla & o_Caulobacterales & f_Caulobacteraceae & g_Brevundimonas & & $0.02 \% \quad 0.02 \%$ & $6.00 \%$ & $\% 0.03^{\circ}$ \\
\hline K_Bacteria & p_Proteobacteria & teobacteria & O_Caulobacterales & f_Caulobacteraceae & g_Brevundimonas & & $0.10 \% \quad 0.09 \%$ & & \\
\hline k_Bacteria & P_Proteobacteria & & o_Caulobacterales & f_Caulobacteraceae & & & $0.00 \% \quad 0.01 \%$ & & 0.019 \\
\hline k_Bacteria & p_Proteobacteria & oteobact & o_Caulobacter & & & & $0.77 \% \quad 0.82 \%$ & & $\% 0.79$ \\
\hline _Bacteria & p_Proteobacteria & & o_Ellin329 & Unclassified_Ellin329 & g_Unclassified_Ellin329 & Unclassified_Ellin3 & $0.01 \% \quad 0.01 \%$ & & $\% 0.00$ \\
\hline k_Bacteria & P_Proteobacteria & $\begin{array}{l}\text { c- }_{-} \\
\text {teobac }\end{array}$ & O_Rhizobiales & f_Aurantimonadaceae & g_Aurar & & $0.00 \% \quad 0.03 \%$ & $6.02 \%$ & $\% 0.00 \%$ \\
\hline Bacteria & p_Proteobacteria & & o_Rhizobiales & f_Beijerinckiaceae & & & $0.00 \% 0.01 \%$ & & (1) 000 \\
\hline E_Bacteria & P_Proteobacteria & & O_Rhizobiales & f_Bradyrhizobiaceae & g_Balneimonas & & $0.01 \% \quad 0.00 \%$ & $6.00 \%$ & $\% 0.00 \%$ \\
\hline k_Bacteria & p_Proteobacteria & & O_Rhizobiales & f_Bradyrhizobiaceae & g_Bosea & genosp. & $0.01 \% 0.00 \%$ & & \\
\hline Bacteria & p_Proteobacteria & oteoba & O_Rhizobiales & f_Bradyrhizobiaceae & & & $0.07 \% \quad 0.09 \%$ & & $\% 0.09$ \\
\hline ¿_Bacteria & P_Proteobacteria & $\begin{array}{c}c_{-} \\
\text {haproteobacteria }\end{array}$ & O_Rhizobiales & f_Bradyrhizobiaceae & & & $0.12 \% \quad 0.11 \%$ & & 作 \\
\hline Bacteria & p_Proteobacteria & haproteoba & o_Rhizobiales & f_Brucellaceae & g_Ochrobactrum & & $2.71 \% 2.58 \%$ & & \\
\hline Bacteria & P_Proteobacteria & & o_Rhizobiales & f_Brucellaceae & g_Pseudochrobactrum & & $0.01 \% \quad 0.00 \%$ & $6.00 \%$ & $\% 0.00 \%$ \\
\hline Bacteria & P_Proteobacteria & hhaproteobacteria & o_Rhizobiales & 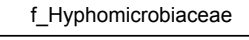 & 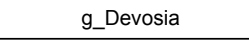 & & $0.03 \% \quad 0.02 \%$ & & \\
\hline
\end{tabular}


Table S3 (continued).

Taxonomy

\begin{tabular}{|c|c|}
\hline k_Bacteria & p_Proteobacteria Alphaproteob \\
\hline k_Bacteria & p_Proteobacteria Alphaproteob \\
\hline k_Bacteria & p_Proteobacteria Alphaproteobacteria \\
\hline k_Bacteria & p_Proteobacteria Alphaproted \\
\hline k_Bacteria & p_Proteobacteria $\frac{c_{-}}{c_{\text {Alphaproteobacteria }}}$ \\
\hline k_Bacteria & p_Proteobacteria $\begin{array}{c}\text { c } \\
\text { Alphaproteobacteria }\end{array}$ \\
\hline k_Bacteria & p_Proteobacteria $\frac{c_{-}}{c_{-}}$Alphaproteobacteria \\
\hline k_Bacteria & p_Proteobacteria $\begin{array}{c}\text { c } \\
\text { Alphaproteobacteria }\end{array}$ \\
\hline k_Bacteria & p_Proteobacteria Alph \\
\hline k_Bacteria & p_Proteobacteria $\begin{array}{c}\mathrm{C}_{-} \\
\text {Alphaproteo }\end{array}$ \\
\hline
\end{tabular}

k_Bacteria p_Proteobacteria Alphaproteobacteria

k_Bacteria p_Proteobacteria Alphaproteobacteria

k_Bacteria p_Proteobacteria Alphaproteobacteria

k_Bacteria p_Proteobacteria Alphaproteobacteria

k_Bacteria p_Proteobacteria Alphaproteobacteria

Alphaproteobacteria O_Rhodobacterales

k_Bacteria P_Proteobacteria Alphaproteobacteria O_Rhodobacterales

k_Bacteria p_Proteobacteria Alphaproteobacteria O_Rhodobacterales

k_Bacteria p_Proteobacteria Alphaproteobacteria O_Rhodobacterales

k_Bacteria p_Proteobacteria Alphaproteobacteria O_Rhodobacterales

k_Bacteria p_Proteobacteria Alphaproteobacteria o_Rhodobacterale

k_Bacteria p_Proteobacteria Alphaproteobacteria O_Rhodobacterales

k_Bacteria p_Proteobacteria Alphaproteobacteria O_Rhodobacterales

k_Bacteria p_Proteobacteria Alphaproteobacteria O_Rhodobacterales

k_Bacteria p_Proteobacteria Alphaproteobacteria O_Rhodobacterales

k_Bacteria p_Proteobacteria Alphaproteobacteria o_Rhodospirillales

k_Bacteria p_Proteobacteria Alphaproteobacteria o_Rhodospirillal

k_Bacteria P_Proteobacteria Alphaproteobacteria O_Rhodospirillale

k_Bacteria p_Proteobacteria Alphaproteobacteria O_Rhodospirillales

k_Bacteria p_Proteobacteria Alphaproteobacteria o_Rhodospirillales

k_Bacteria p_Proteobacteria Alphaproteobacteria o_Rhodospirillales

k_Bacteria p_Proteobacteria Alphaproteobacteria O_Rhodospirillales

k_Bacteria p_Proteobacteria Alphaproteobacteria o_Rickettsiales

k_Bacteria p_Proteobacteria Alphaproteobacteria O_Rickettsiales

k_Bacteria p_Proteobacteria Alphaproteobacteria o_Rickettsiale

k_Bacteria P_Proteobacteria Alphaproteobacteria o_Rickettsiales

o_mitochondria

k_Bacteria P_Proteobacteria Alphaproteobacteria O_Sphingomonadales f_Erythrobacteraceae

k_Bacteria p_Proteobacteria Alphaproteobacteria ${ }^{\circ}$ _Sphingomonadales f_Sphingomonadaceae

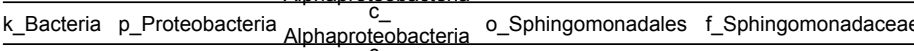

k_Bacteria p_Proteobacteria Alphaproteobacteria ${ }^{\circ}$ _Sphingomonadales f_Sphingomonadacea

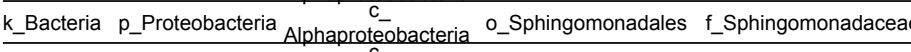

k_Bacteria p_Proteobacteria Alphaproteobacteria o_Sphingomonadales f_Sphingomonadaceae

k_Bacteria p_Proteobacteria Alphaproteobacteria O_Sphingomonadales f_Sphingomonadaceae

k_Bacteria p_Proteobacteria Alphaproteobacteria O_Sphingomonadales f_Sphingomonadaceae

k_Bacteria p_Proteobacteria Alphaproteobacteria ${ }^{\circ}$ _Sphingomonadales f_Sphingomonadaceae

k_Bacteria p_Proteobacteria Alphaproteobacteria O_Sphingomonadales f_Sphingomonadacea

k Bacteria $p$ Proteobacteria $c_{-}$o Sphingomonadales f_Unclassified

Alphaproteobacteria - O_Unclassified_ Sphingomonadales

k_Bacteria P_Proteobacteria Alphaproteobacteria Alphaproteobacteria Alphaproteobacteria

k_Bacteria p_Proteobacteria $\begin{gathered}\text { C_- } \\ \text { Betaproteobacteria }\end{gathered}$

k_Bacteria p_Proteobacteria $\begin{gathered}\text { C_ } \\ \text { Betaproteobacteria }\end{gathered}$

k_Bacteria p_Proteobacteria $\begin{gathered}\text { C_ }_{\text {B }} \\ \text { Betaproteobacteria }\end{gathered}$ o_Burkholderiales $\quad$ f_Burkholderiaceae

k_Bacteria p_Proteobacteria $\begin{gathered}\text { C_ } \\ \text { Betaproteobacteria o_Burkholderiales f_Burkholderiaceae }\end{gathered}$

k_Bacteria p_Proteobacteria Betaproteobacteria o_Burkholderiales f_Burkholderiaceae

k_Bacteria p_Proteobacteria $\begin{gathered}\text { C_ } \\ \text { Betaproteobacteria o_Burkholderiales f_Comamonadaceae }\end{gathered}$

k_Bacteria p_Proteobacteria $\begin{gathered}\text { C_ } \\ \text { Betaproteobacteria }\end{gathered}$
g_Unclassified_

Hyphomicrobiaceae

g_Methylobacterium

Methylobacterium

g_Methylobacterium

g_Unclassified_

Methylobacteriaceae

Methylocystaceae

g_Aminobacter

g_Aquamicrobium

g_Phyllobacterium

g_Unclassified_

Phyllobacteriaceae

g_Agrobacterium

g_Unclassified_
Rhizobiaceae
g_Unclassified_

Rhizobiales

g_Labrys

g_Xanthobacter

g_Unclassified

Hyphomonadaceae

g_Anaerospora

g_Loktanella

g_Oceanicella

g_Octadecabacter

g_Paracoccus

g_Paracoccus

g_Rhodobacter

g_Rubellimicrobium

g_Unclassified_

Rhodobacteraceae

g_Gluconobacter

g_Unclassified

Acetobacteraceae

g_Azospirillum

g_Azospirillum

g_Unclassified

Rhodospirillaceae
g_Unclassified_

Rhodospirillales
g Unclassified

Holosporaceae

g_Carludovica

g_Nelumbo

g_Zea

g_Unclassified

Erythrobacteraceae

g_Blastomonas

g_Kaistobacter

g_Novosphingobium

g_Novosphingobium

g_Sphingobium

g_Sphingomonas

g_Sphingomonas

g_Sphingopyxis

g_Unclassified

Sphingomonadacea

Sphingomonadales

Alphaproteobacteri

g_Achromobacter

g_Sutterella

g_Burkholderia

g_Lautropia

g_Unclassified

Burkholderiaceae

g_Comamonas

g_Delftia
Relative aboundance of taxa \begin{tabular}{llll} 
M1 & M2 & M3 & M4 \\
\hline
\end{tabular}

$\begin{array}{llllll}\text { S_Unclassified__ } & 0.02 \% & 0.02 \% & 0.00 \% & 0.00 \%\end{array}$

$\begin{array}{lllll}\text { Hyphomicrobiaceae } & 0.00 \% & 0.00 \%\end{array}$

$\begin{array}{lllll}\text { komagatae } & & 0.01 \% & 0.00 \% & 0.01 \%\end{array}$ $\begin{array}{llllll}\text { organophilum } & 0.07 \% & 0.06 \% & 0.00 \% & 0.06 \%\end{array}$ $\begin{array}{lllll}\text { Methylobacterium } & 0.07 \% & & & \\ \text { s_Unclassified_- } & 0.11 \% & 0.12 \% & 0.01 \% & 0.10 \%\end{array}$ \begin{tabular}{llllll} 
Methylobacteriaceae & $0.11 \%$ & $0.12 \%$ & $0.01 \%$ & $0.10 \%$ \\
\hline s Unctasified & & $0.00 \%$ & $0.01 \%$ & $0.00 \%$ & $0.00 \%$
\end{tabular} $\begin{array}{lllllll}\text { s_Unclassified_- } & 0.00 \% & 0.01 \% & 0.00 \% & 0.00 \%\end{array}$ \begin{tabular}{llllll} 
Methylocystaceeae & $0.00 \%$ & $0.01 \%$ & $0.00 \%$ & $0.00 \%$ \\
\hline S_Unclassified_ & $0.03 \%$ & $0.03 \%$ & $0.00 \%$ & $0.02 \%$
\end{tabular} \begin{tabular}{lllll} 
Anminobacter $^{-}$ & $0.03 \%$ & $0.03 \%$ & $0.00 \%$ & $0.02 \%$ \\
\hline Aquamicrobium_- & $0.00 \%$ & $0.00 \%$ & $0.00 \%$ & $0.01 \%$
\end{tabular} s Unclassified

$\begin{array}{lllllll}\text { Phyllobacterium } & 0.02 \% & 0.02 \% & 0.00 \% & 0.02 \%\end{array}$

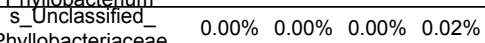
$\begin{array}{lllll}\text { S_Unclassified_ } & 0.13 \% & 0.15 \% & 0.03 \% & 0.18 \%\end{array}$ $\begin{array}{lllll}\text { Agrobacterium } & 0.13 \% & 0.15 \% & 0.03 \% & 0.18 \% \\ \text { s_Unclassified_- } & 0.03 \% & 0.00 \% & 0.00 \% & 0.00 \%\end{array}$ $\begin{array}{llllll}\text { S_Unizobiaceae }_{\text {SUnsified_- }} & 0.10 \% & 0.09 \% & 0.00 \% & 0.12 \%\end{array}$ \begin{tabular}{lllll}
\hline Rhizobiales & $0.10 \%$ & $0.09 \%$ & $0.00 \%$ & $0.12 \%$ \\
\hline nnclassified_Labrys & $0.00 \%$ & $0.00 \%$ & $0.00 \%$ & $0.00 \%$
\end{tabular} $\begin{array}{lllllll}\text { S_Unclassified_- } & 0.03 \% & 0.04 \% & 0.00 \% & 0.01 \%\end{array}$ \begin{tabular}{llllll} 
Xanthobacter & $0.03 \%$ & $0.04 \%$ & $0.00 \%$ & $0.01 \%$ \\
\hline sUnclassified_ & $0.00 \%$ & $0.00 \%$ & $0.00 \%$ & $0.06 \%$
\end{tabular} \begin{tabular}{lllll} 
Hyphomonadaceae & $0.00 \%$ & $0.00 \%$ & $0.00 \%$ & $0.06 \%$ \\
\hline S_Unclassified_ & $0.02 \%$ & $0.00 \%$ & $0.00 \%$ & $0.00 \%$
\end{tabular} \begin{tabular}{lllll} 
Anaerospora $_{\text {Anclassified_- }}$ & $0.02 \%$ & $0.00 \%$ & $0.00 \%$ & $0.00 \%$ \\
\hline _Uncla & $0.05 \%$ & $0.00 \%$ & $0.00 \%$ & $0.00 \%$
\end{tabular} $\begin{array}{llllll}\text { Loktanella } & & 0.00 \% & 0.00 \%\end{array}$ \begin{tabular}{llllll}
\hline S_Unclassified_- & $0.02 \%$ & $0.01 \%$ & $0.00 \%$ & $0.16 \%$
\end{tabular} Octadecabacter

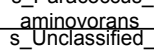
\begin{tabular}{lllllll}
\hline Paracoccus & $0.03 \%$ & $0.01 \%$ & $0.00 \%$ & $0.02 \%$
\end{tabular} $\begin{array}{llllll}\text { S_Uaracoccus } & & 0.000\end{array}$ \begin{tabular}{lllll} 
Rhodobacter $_{\text {R_Unclassified_- }}$ & $0.06 \%$ & $0.02 \%$ & $0.00 \%$ & $0.00 \%$ \\
\hline & $0.00 \%$ & $0.01 \%$ & $0.00 \%$ & $0.00 \%$
\end{tabular} \begin{tabular}{lllll} 
Rubellimicrobium & $0.00 \%$ & $0.01 \%$ & $0.00 \%$ & $0.00 \%$ \\
\hline s_Unclassified_- & $0.04 \%$ & $0.02 \%$ & $0.00 \%$ & $0.19 \%$
\end{tabular} Rhodobacteraceae $\begin{array}{llllll}\text { s_Unclassified_- } & 0.00 \% & 0.00 \% & 0.00 \% & 0.00 \%\end{array}$ s_unclassified GluconobacterAcetobacteraceae massiliensis Azospirillum Rhodospirillaceae Rhodospirillales Holosporaceae $\begin{array}{llllll}\text { s_Carludovica_palmata } & 0.00 \% & 0.00 \% & 0.00 \% & 0.00 \%\end{array}$ $\begin{array}{llllll}\text { s_Nelumbo_nucifera } & 0.02 \% & 0.00 \% & 0.00 \% & 0.00 \%\end{array}$ $\begin{array}{lllllll}\text { s_Zea_luxurians } & 0.00 \% & 0.01 \% & 0.00 \% & 0.01 \%\end{array}$ \begin{tabular}{lllllll}
\hline S_Unclassified_- & $0.03 \%$ & $0.00 \%$ & $0.00 \%$ & $0.00 \%$
\end{tabular} \begin{tabular}{lllll} 
Erythrobacteraceae & $0.03 \%$ & $0.00 \%$ & $0.00 \%$ & $0.00 \%$ \\
\hline S_Blastomonas & $0.01 \%$ & $0.00 \%$ & $0.00 \%$ & $0.01 \%$
\end{tabular} s_Unclasiasified_

\begin{tabular}{lllllll} 
Kaistobacter $^{-}$ & $0.00 \%$ & $0.01 \%$ & $0.00 \%$ & $0.02 \%$ \\
\hline
\end{tabular} $\begin{array}{lllllll} & \\ \text { stygium } & 0.02 \% & 0.04 \% & 0.00 \% & 0.03 \%\end{array}$ s_unclaium \begin{tabular}{lllllll} 
Novosphingobium & $0.01 \%$ & $0.03 \%$ & $0.00 \%$ & $0.02 \%$ \\
\hline
\end{tabular}

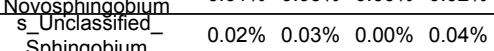
\begin{tabular}{llllll} 
Sphingobium & $0.02 \%$ & $0.03 \%$ & $0.00 \%$ & $0.04 \%$ \\
\hline Sphingomonas_ & $1.34 \%$ & $1.83 \%$ & $0.01 \%$ & $1.55 \%$
\end{tabular} s_Unotifigens

Sphingomonas Sphingopyxis $\begin{array}{lllll}0.12 \% & 0.08 \% & 0.00 \% & 0.09 \%\end{array}$ $\begin{array}{lllll}0.00 \% & 0.00 \% & 0.00 \% & 0.00 \%\end{array}$

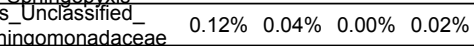
\begin{tabular}{llllll}
\hline sphingomonadaceae & $0.12 \%$ & $0.04 \%$ & $0.00 \%$ & $0.02 \%$ \\
\hline s_Unclassified_ & $0.05 \%$ & $0.04 \%$ & $0.00 \%$ & $0.01 \%$
\end{tabular} \begin{tabular}{ccccc} 
Sphingomonadales & $0.05 \%$ & $0.04 \%$ & $0.00 \%$ & $0.01 \%$ \\
\hline s_Unclassified- & $0.06 \%$ & $0.03 \%$ & $0.00 \%$ & $0.01 \%$
\end{tabular} \begin{tabular}{ccccc} 
Alphaproteobacteria & $0.06 \%$ & $0.03 \%$ & $0.00 \%$ & $0.01 \%$ \\
\hline s_Unclassified_- & $0.00 \%$ & $0.00 \%$ & $0.00 \%$ & $0.00 \%$
\end{tabular} Achromobacter

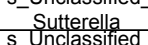
$\begin{array}{lllll}0.00 \% & 0.01 \% & 0.00 \% & 0.11 \%\end{array}$ $\begin{array}{llllll} & \text { SUnclassified_- } & 14.16 \% & 14.32 \% & 0.10 \% & 11.89 \%\end{array}$ Burkholderia S Unnclassia $\begin{array}{lllllllllllllllll} & 0.01 \% & 0.01 \% & 0.00 \% & 0.00 \%\end{array}$ s_Unclassified Comamonas

$\begin{array}{lllll}0.01 \% & 0.01 \% & 0.00 \% & 0.00 \%\end{array}$

$\begin{array}{lllll}\text { s_Unclassified_Delftia } & 0.03 \% & 0.04 \% & 0.00 \% & 0.03 \%\end{array}$ 
Table S3 (continued).

Taxonomy

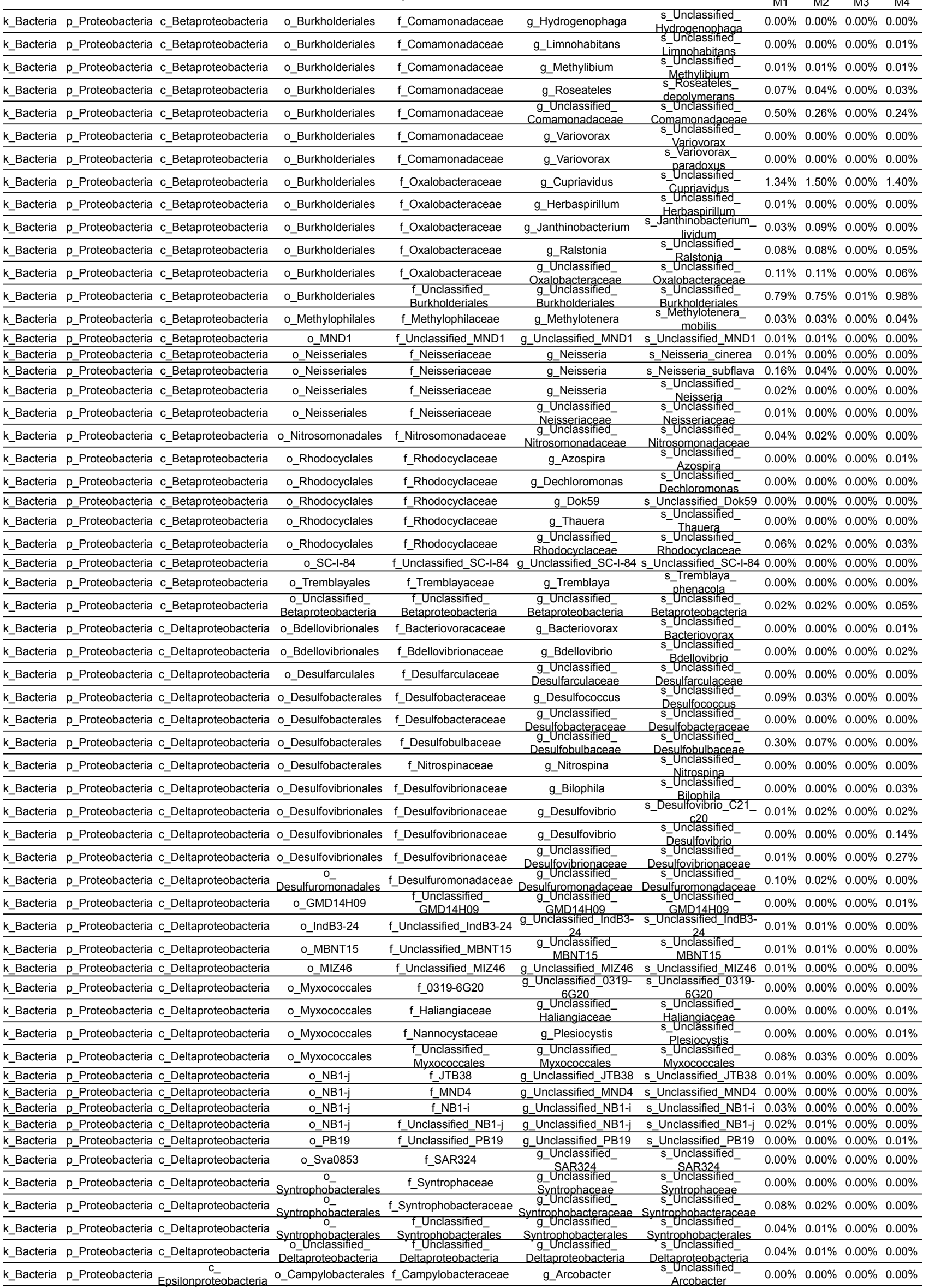

Relative aboundance of taxa Relative aboundance of taxa
M1 M2 M3 M4 $0.00 \%$ $\begin{array}{lllllllll}0.01 \% & 0.01 \% & 0.00 \% & 0.01 \%\end{array}$ (n)

.

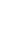


Taxonomy

\begin{tabular}{|c|c|c|c|c|c|c|c|c|c|}
\hline & \multirow{2}{*}{$\frac{\mathrm{M} 1}{0.00 \%}$} & \multirow{2}{*}{$\frac{\mathrm{M} 2}{0.00 \%}$} & \multirow{2}{*}{$\frac{\mathrm{M} 3}{0.00 \%}$} & \multirow{2}{*}{$\begin{array}{c}\text { M4 } \\
2.73 \% \\
\end{array}$} \\
\hline Bacteria & $\begin{array}{c}\text { P_Epsilon- } \\
\text { bacteraeota }\end{array}$ & o_Campylobacterales & f_Sulfurovaceae & g_Sulfurovum & $\begin{array}{l}\text { S_Unclassified__ } \\
\text { Sulfurovum }\end{array}$ & & & & \\
\hline K_Bacteria & p_Proteobacteria & c_Epsilonproteobacteria o_Campylobacterales & f_Helicobacteraceae & $\begin{array}{l}\text { g_Unclassified } \\
\text { Helicobacteraceae }\end{array}$ & $\begin{array}{l}\text { S_Unclassified_- } \\
\text { Helicobacteraceae }\end{array}$ & $0.07 \%$ & $0.01 \%$ & $0.00 \%$ & $6.41 \%$ \\
\hline Bacteria & p_Proteobacteria & c_Gammaproteobacteria & f_[Marinicellaceae] & g_Marinicella & & $0.00 \%$ & $0.00 \%$ & $0.00 \%$ & $0.18 \%$ \\
\hline K_Bacteria & p_Proteobacteria & c_Gammaproteobacteria & f_[Marinicellaceae] & $\begin{array}{l}\text { g_Un } \\
\text { [Marin }\end{array}$ & alfied & $0.16 \%$ & $0.07 \%$ & $0.00 \%$ & $0.00 \%$ \\
\hline Bacteria & p_Proteobacteria & c_Gammaproteobacteria o_Aeromonadales & f_Aeromonadaceae & $\begin{array}{l}\text { g_Unclassified - } \\
\text { Aeromonadaceae }\end{array}$ & A_Un & $0.07 \%$ & $0.03 \%$ & $0.00 \%$ & $0.00 \%$ \\
\hline Bacteria & p_Proteobacteria & c_Gammaproteobacteria o_Alteromonadales & f_[Chromatiaceae] & g_Rheinheimera & ied_- & $0.00 \%$ & $0.00 \%$ & $0.00 \%$ & $0.01 \%$ \\
\hline K_Bacteria & p_Proteobacteria & c_Gammaproteobacteria o_Alteromonadales & f_Alteromonadaceae & g_Alteromonas & $\begin{array}{l}\text { s_Unclassified_- } \\
\text { Alteromonas }\end{array}$ & $0.01 \%$ & $0.00 \%$ & $0.00 \%$ & $0.00 \%$ \\
\hline Bacteria & p_Proteobacteria & c_Gammaproteobacteria o_Alteromonadales & f_Alteromonadaceae & g_Cellvibrio & ified_ & $0.00 \%$ & $0.00 \%$ & $0.00 \%$ & $0.01 \%$ \\
\hline k_Bacteria & p_Proteobacteria & c_Gammaproteobacteria o_Alteromonadales & f_Alteromonadaceae & $\begin{array}{c}\text { g_Unclassified_ } \\
\text { Alteromonadaceae }\end{array}$ & $\begin{array}{l}\text { S_Unclassified_ } \\
\text { Alteromonadaceae }\end{array}$ & $0.00 \%$ & $0.00 \%$ & $0.00 \%$ & $0.00 \%$ \\
\hline Bacteria & p_Proteobacteria & c_Gammaproteobacteria o_Alteromonadales & f_HTCC2188 & g_H & ied_HTCC & $0.01 \%$ & $0.00 \%$ & $0.00 \%$ & $0.00 \%$ \\
\hline Bacteria & Proteobacteria & c_Gammaproteobacteria o_Alteromonadales & & g_Unclassified_OM60 & ied_OM60 & $0.04 \%$ & $0.01 \%$ & $0.00 \%$ & $0.00 \%$ \\
\hline k_Bacteria & p_Proteobacteria & c_Gammaproteobacteria o_Alteromonadales & f_Psychromonadaceae & g_Psychromonas & $\begin{array}{l}\text { ssified__ } \\
\text { monas }\end{array}$ & $0.00 \%$ & $0.00 \%$ & $0.00 \%$ & $0.00 \%$ \\
\hline __Bacteria & p_Proteobacteria & c_Gammaproteobacteria o_Cardiobacteriales & f_Cardiobacteriaceae & g_Cardiobacterium & actified & $0.00 \%$ & $0.00 \%$ & $0.00 \%$ & $0.00 \%$ \\
\hline Bacteria & _Proteobacteria & c_Gammaproteobacteria & f_Chromatiaceae & $\begin{array}{l}\text { g_Unc } \\
\text { Chrom }\end{array}$ & sified & $0.00 \%$ & $0.00 \%$ & $0.00 \%$ & $0.01 \%$ \\
\hline __Bacteria & p_Proteobacteria & c_Gammaproteobacteria & $\stackrel{f}{f_{-}}$ & $\begin{array}{l}\text { g_Unc } \\
\text { tothiorh }\end{array}$ & sified_- & $0.00 \%$ & $0.01 \%$ & $0.00 \%$ & $0.00 \%$ \\
\hline Bacteria & p_Proteobacteria & c_Gammaproteobacteria & $\begin{array}{l}\text { f_Unclassified_- } \\
\text { Chromatiales }\end{array}$ & $\begin{array}{l}\text { 9_Unclassified_- } \\
\text { Chromatiales }\end{array}$ & $\begin{array}{l}\text { Sified_ } \\
\text { tiales }\end{array}$ & $0.27 \%$ & $0.11 \%$ & $0.00 \%$ & $0.02 \%$ \\
\hline Bacteria & p_Proteobacteria & c_Gammaproteobacteria o_Enterobacteriales & f_Enterobacteriaceae & g_Citrobacter & $\begin{array}{l}\text { sified_ } \\
\text { cter_- }\end{array}$ & $0.23 \%$ & $1.58 \%$ & $0.95 \%$ & $0.27 \%$ \\
\hline Bacteria & p_Proteobacteria & c_Gammaproteobacteria o_Enterobacteriales & f_Enterobacteriaceae & g_Enterobacter & $\begin{array}{l}\text { bacter_- } \\
\text { viae }\end{array}$ & $1.41 \%$ & $2.39 \%$ & $15.61 \%$ & $0.93 \%$ \\
\hline Bacteria & p_Proteobacteria & c_Gammaproteobacteria o_Enterobacteriales & f_Enterobacteriaceae & g_Enterobacter & $\begin{array}{l}\text { aacter_- } \\
\text { chei }\end{array}$ & $0.29 \%$ & $0.13 \%$ & $0.11 \%$ & $0.03 \%$ \\
\hline Bacteria & p_Proteobacteria & C_Gammaproteobacteria o_Enterobacteriales & f_Enterobacteriaceae & g_Enterobacter & s_Enterobacter_ludwigii & $0.00 \%$ & $0.00 \%$ & $0.01 \%$ & $0.00 \%$ \\
\hline Bacteria & p_Proteobacteria & c_Gammaproteobacteria o_Enterobacteriales & f_Enterobacteriaceae & g_Enterobacter & $\begin{array}{l}\text { bacter_ } \\
\text { nsis }\end{array}$ & $18.09 \%$ & $6.90 \%$ & $5.12 \%$ & $2.58 \%$ \\
\hline _Bacteria & p_Proteobacteria & c_Gammaproteobacteria o_Enterobacteriales & f_Enterobacteriaceae & g_Enterobacter & $\begin{array}{l}\text { sifified_- } \\
\text { acter }\end{array}$ & $0.03 \%$ & $0.11 \%$ & $0.59 \%$ & $0.01 \%$ \\
\hline Bacteria & p_Proteobacteria & C_Gammaproteobacteria o_Enterobacteriales & f_Enterobacteriaceae & g_Erwinia & ied_Erwinia & $0.01 \%$ & $0.03 \%$ & $0.24 \%$ & $0.01 \%$ \\
\hline Bacteria & p_Proteobacteria & c_Gammaproteobacteria o_Enterobacteriales & f_Enterobacteriaceae & g_Escherichia & ia_blattae & $0.00 \%$ & $0.00 \%$ & $0.00 \%$ & $0.00 \%$ \\
\hline k_Bacteria & p_Proteobacteria & c_Gammaproteobacteria o_Enterobacteriales & f_Enterobacteriaceae & g_Klebsiella & $\begin{array}{l}\text { sified_- } \\
\text { ella }\end{array}$ & $0.01 \%$ & $0.15 \%$ & $0.44 \%$ & $0.15 \%$ \\
\hline Bacteria & p_Proteobacteria & c_Gammaproteobacteria o_Enterobacteriales & f_Ente & g_Mc & morganii & $0.06 \%$ & $0.48 \%$ & $0.13 \%$ & $0.04 \%$ \\
\hline k_Bacteria & p_Proteobacteria & c_Gammaproteobacteria o_Enterobacteriales & f_Enterobacteriaceae & g_Morganella & $\begin{array}{r}\mathrm{S}_{\mathrm{N}} \mathrm{Ur} \\
\mathrm{MC}\end{array}$ & $0.00 \%$ & $0.04 \%$ & $0.02 \%$ & $0.00 \%$ \\
\hline Bacteria & p_Proteobacteria & c_Gammaproteobacteria o_Enterobacteriales & f_Enterobacteriaceae & g_Pantoea & $\begin{array}{l}\text { toea }_{-} \\
\text {erans }\end{array}$ & $0.00 \%$ & $0.01 \%$ & $0.00 \%$ & $0.00 \%$ \\
\hline Bacteria & Proteobacteria & C_Gammaproteobacteria o_Enterobacteriales & f_Ente & g_P & ananatis & $0.00 \%$ & $0.00 \%$ & $0.00 \%$ & $0.00 \%$ \\
\hline Bacteria & p_Proteobacteria & C_Gammaproteobacteria o_Enterobacteriales & f_Enterobacteriaceae & g_Proteus & d_Proteus & $0.00 \%$ & $0.00 \%$ & $0.01 \%$ & $0.00 \%$ \\
\hline k_Bacteria & p_Proteobacteria & c_Gammaproteobacteria o_Enterobacteriales & f_Enterobacteriaceae & g_Providencia & $\frac{\mathrm{P}^{2}}{\mathrm{~s}^{2} \mathrm{c}}$ & $0.00 \%$ & $0.02 \%$ & $0.01 \%$ & $0.00 \%$ \\
\hline Bacteria & p_Proteobacteria & c_Gammaproteobacteria o_Enterobacteriales & f_Enterobacteriaceae & g_Salmonella & illed_- & $0.01 \%$ & $0.00 \%$ & $0.03 \%$ & $0.00 \%$ \\
\hline Bacteria & p_Proteobacteria & C_Gammaproteobacteria o_Enterobacteriales & f_Enterobacteriaceae & g_Serratia & marcescens & $0.02 \%$ & $0.07 \%$ & $0.13 \%$ & $0.02 \%$ \\
\hline Bacteria & Proteobacteria & C_Gammaproteobacteria o_Enterobacteriales & f_Enter & & ia_ureilytica & $0.00 \%$ & $0.01 \%$ & $0.11 \%$ & $0.00 \%$ \\
\hline Bacteria & Proteobacteria & C_Gammaproteobacteria o_Enter & f_Ente & & ed_Serratia & $0.23 \%$ & $0.26 \%$ & $0.11 \%$ & $0.40 \%$ \\
\hline Bacteria & p_Proteobacteria & c_Gammaproteobacteria o_Enterobacteriales & f_Enterobacteriaceae & g_Trabulsiella & lla_farmeri & $0.00 \%$ & $0.03 \%$ & $0.00 \%$ & $0.01 \%$ \\
\hline k_Bacteria & p_Proteobacteria & c_Gammaproteobacteria o_Enterobacteriales & f_Enterobacteriaceae & g_Trabulsiella & $\begin{array}{l}\text { iffied_ } \\
\text { ella }\end{array}$ & $0.00 \%$ & $0.01 \%$ & $0.00 \%$ & $0.00 \%$ \\
\hline Bacteria & p_Proteobacteria & c_Gammaproteobacteria o_Enterobacteriales & f_Enterobacteriaceae & g_Escherichia-Shigella & $\begin{array}{l}\text { Sified_- } \\
\text { Shigella }\end{array}$ & $0.06 \%$ & $0.35 \%$ & $0.07 \%$ & $4.20 \%$ \\
\hline K_Bacteria & p_Proteobacteria & c_Gammaproteobacteria o_Enterobacteriales & f_Enterobacteriaceae & $\begin{array}{l}\text { 9_Unclassified_- } \\
\text { Enterobacteriaceae }\end{array}$ & $\begin{array}{l}\text { S_U } \\
\text { Entero }\end{array}$ & $0.90 \%$ & $2.84 \%$ & $6.13 \%$ & $1.57 \%$ \\
\hline __Bacteria & p_Proteobacteria & c_Gammaproteobacteria o_Enterobacteriales & f_Enterobacteriaceae & g_Xenorhabdus & $\stackrel{\mathrm{b}}{\mathrm{s} \mathrm{Xe}_{\mathrm{H}}}$ & $0.00 \%$ & $0.00 \%$ & $0.00 \%$ & $0.00 \%$ \\
\hline Bacteria & p_Proteobacteria & c_Gammaproteobacteria & f_Unclassified_HOC36 & g_Unclass & d_HOC36 & $0.00 \%$ & $0.00 \%$ & $0.00 \%$ & $0.00 \%$ \\
\hline k_Bacteria & p_Proteobacteria & c_Gammaproteobacteria & f_HTCC2089 & $\begin{array}{c}\text { g_Unclassified_ } \\
\text { HTCC2089 }\end{array}$ & 2089 & $0.03 \%$ & $0.01 \%$ & $0.00 \%$ & $0.00 \%$ \\
\hline Bacteria & p_Proteobacteria & c_Gammaproteobacteria & f_Coxiellaceae & g_Aquicella & & $0.00 \%$ & $0.00 \%$ & $0.00 \%$ & $0.01 \%$ \\
\hline B_Bacteria & p_Proteobacteria & c_Gammaproteobacteria & f_Coxiellaceae & g_Rickettsiella & Sified_- & $0.07 \%$ & $0.03 \%$ & $6.43 \%$ & $0.06 \%$ \\
\hline __Bacteria & p_Proteobacteria & c_Gammaproteobacteria & $\begin{array}{l}\text { f_Unclassified_ } \\
\text { Legionellales }\end{array}$ & $\begin{array}{l}\text { g_Uncla } \\
\text { Legion }\end{array}$ & ales & $0.00 \%$ & $0.00 \%$ & $0.00 \%$ & $0.00 \%$ \\
\hline B_Bacteria & p_Proteobacteria & C_Gammaproteobacteria o_Methylococcales & f_Methylomonaceae & $\begin{array}{l}\text { g_Marine } \\
\text { athylotrophic Group }\end{array}$ & Methylo & $0.00 \%$ & $0.00 \%$ & $0.00 \%$ & $4.44 \%$ \\
\hline k_Bacteria & p_Proteobacteria & c_Gammaproteobacteria o_Methylococcales & $\begin{array}{l}\text { f_Unclassified_ } \\
\text { Methylococcales }\end{array}$ & $\begin{array}{l}\text { 9.Unclassified } \\
\text { Methylococcales }\end{array}$ & $\begin{array}{l}\text { s.Un } \\
\text { Methy } \\
\text { sun }\end{array}$ & $0.00 \%$ & $0.00 \%$ & $0.00 \%$ & $10.90 \%$ \\
\hline Bacteria & p_Proteobacteria & c_Gammaproteobacteria & f_Pasteurellaceae & g_Aggregatibacter & $\mathrm{s}$ & $0.01 \%$ & $0.00 \%$ & $0.00 \%$ & $0.00 \%$ \\
\hline K_Bacteria & p_Proteobacteria & c_Gammaproteobacteria & f_Pasteurellaceae & g_Gallibacterium & & $0.01 \%$ & $0.00 \%$ & $0.00 \%$ & $0.00 \%$ \\
\hline Bacteria & p_Proteobacteria & c_Gammaproteobacteria & f_Pasteurellaceae & hilus & & $0.17 \%$ & $0.03 \%$ & $0.00 \%$ & $0.00 \%$ \\
\hline B_Bacteria & p_Proteobacteria & C_Gammaproteobacteria & & $\begin{array}{l}\text { g_Unclassified_- } \\
\text { Pasteurellales }\end{array}$ & & $0.20 \%$ & $0.11 \%$ & $0.00 \%$ & $0.04 \%$ \\
\hline k_Bacteria & p_Proteobacteria & c_Gammaproteobacteria o_Pseudomonadales & f_Moraxellaceae & g_Acinetobacter & & $0.01 \%$ & $0.02 \%$ & $0.05 \%$ & $0.02 \%$ \\
\hline Bacteria & p_Proteobacteria & c_Gammaproteobacteria o_Pseudomonadales & f_Moraxellaceae & g_Acinetobacter & ii & $0.01 \%$ & $0.05 \%$ & $0.00 \%$ & $0.01 \%$ \\
\hline Bacteria & p_Proteobacteria & C_Gammaproteobacteria o_Pseudomonadales & f_Moraxellaceae & g_Acinetobacter & reIwoffii & $0.06 \%$ & $0.00 \%$ & $0.00 \%$ & $0.00 \%$ \\
\hline k_Bacteria & p_Proteobacteria & c_Gammaproteobacteria o_Pseudomonadales & f_Moraxellaceae & g_Acinetobacter & & $0.01 \%$ & $0.00 \%$ & $0.00 \%$ & $0.00 \%$ \\
\hline K_Bacteria & p_Proteobacteria & c_Gammaproteobacteria o_Pseudomonadales & f_Moraxellaceae & g_Acinetobacter & & $4.50 \%$ & $6.94 \%$ & $0.13 \%$ & $0.25 \%$ \\
\hline k_Bacteria & p_Proteobacteria & c_Gammaproteobacteria o_Pseudomonadales & f_Moraxellaceae & g_Enhydrobacter & & $0.00 \%$ & $0.00 \%$ & $0.00 \%$ & $0.00 \%$ \\
\hline k_Bacteria & p_Proteobacteria & c_Gammaproteobacteria o_Pseudomonadales & f_Moraxellaceae & g_Psychrobacter & & $0.00 \%$ & $0.00 \%$ & $0.00 \%$ & $0.01 \%$ \\
\hline Bacteria & p_Proteobacteria & c_Gammaproteobacteria o_Pseudomonadales & f_Moraxellaceae & icter & & $0.00 \%$ & $0.00 \%$ & $0.00 \%$ & $0.00 \%$ \\
\hline k_Bacteria & p_Proteobacteria & c_Gammaproteobacteria o_Pseudomonadales & f_Moraxellaceae & $\mathrm{ed}_{-}$ & $\begin{array}{l}\text { sified_- } \\
\text { ceae }\end{array}$ & $0.40 \%$ & $0.05 \%$ & $0.01 \%$ & $0.00 \%$ \\
\hline
\end{tabular}


Table S3 (continued)

\section{Taxonomy}

\begin{tabular}{|c|c|c|c|c|c|c|c|c|c|c|}
\hline \multirow[b]{2}{*}{ k_Bacteria } & \multirow{2}{*}{\multicolumn{2}{|c|}{ p_Proteobacteria c_Gammaproteobacteria }} & \multicolumn{3}{|c|}{ 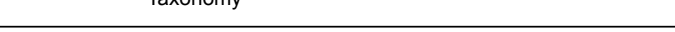 } & \multicolumn{5}{|c|}{$\begin{array}{llll}\text { M1 } & \text { M2 } & \text { M3 } & \text { M4 } \\
\end{array}$} \\
\hline & & & o_Pseudomonadales & f_Pseudomonadaceae & g_Pseudomonas & $\begin{array}{l}\text { S_Pseudomonas_ } \\
\text { viridiflava }\end{array}$ & $0.00 \%$ & $0.00 \%$ & $0.00 \%$ & $0.00 \%$ \\
\hline & p_Proteobacteria & C_Gammaproteobacteria & o_Pseudomonadales & f_Pseudomonadaceae & g_Pseudomonas & $\begin{array}{l}\text { Hassified__ } \\
\text { domonas }\end{array}$ & $0.11 \%$ & $0.13 \%$ & $0.00 \%$ & $0.18 \%$ \\
\hline k_Bacteria & p_Proteobacteria & C_Gammaproteobacteria & O_Pseudomonadales & f_Pseudomonadaceae & $\begin{array}{c}\text { g_Unclassified__ } \\
\text { Pseudomonadaceae }\end{array}$ & tassified_- & $0.03 \%$ & $0.55 \%$ & $0.00 \%$ & $0.02 \%$ \\
\hline Bacteria & p_Proteobacteria & C_Gammaproteobacteria & o_Thiohalorhabdales & $\begin{array}{c}\text { fonclassified } \\
\text { Thiohalorhabdales }\end{array}$ & $\begin{array}{c}\text { g_Unclassified } \\
\text { Thiohalorhabdales }\end{array}$ & $\begin{array}{c}\text { S_Unclassified } \\
\text { Thiohalorhabdales }\end{array}$ & $0.01 \%$ & $0.00 \%$ & $0.00 \%$ & $0.07 \%$ \\
\hline Bacteria & p_Proteobacteria & c_Gammaproteobacteria & o_Thiotrichales & f_Piscirickettsiaceae & $\begin{array}{l}\text { g_Unclassified_- } \\
\text { Piscirickettsiaceae }\end{array}$ & $\begin{array}{l}\text { s_Unclassified } \\
\text { Piscirickettsiaceae }\end{array}$ & $0.62 \%$ & $0.32 \%$ & $0.00 \%$ & $0.88 \%$ \\
\hline Bacteria & p_Proteobacteria & c_Gammaproteobacteria & O_Thiotrichales & f_Thiotrichaceae & g_B46 & s_Unclassified_B46 & $0.00 \%$ & $0.00 \%$ & $0.00 \%$ & $7.87 \%$ \\
\hline Bacteria & p_Proteobacteria & C_Gammaproteobacteria & O_Thiotrichales & f_Thiotrichaceae & g_CF-26 & s_Unclassified_CF-26 & $0.00 \%$ & $0.00 \%$ & $0.00 \%$ & $0.05 \%$ \\
\hline B_Bacteria & p_Proteobacteria & c_Gammaproteobacteria & o_Thiotrichales & f_Thiotrichaceae & g_Cocleimonas & $\begin{array}{l}\text { s_Unclassified_ } \\
\text { Cocleimonas }\end{array}$ & $0.00 \%$ & $0.00 \%$ & $0.00 \%$ & $2.43 \%$ \\
\hline Bacteria & p_Proteobacteria & c_Gammaproteobacteria & o_Thiotrichales & f_Thiotrichaceae & g_E8 & s_Unclassified_E8 & $0.00 \%$ & $0.00 \%$ & $0.00 \%$ & $6.14 \%$ \\
\hline k_Bacteria & p_Proteobacteria & c_Gammaproteobacteria & o_Thiotrichales & f_Thiotrichaceae & $\begin{array}{l}\text { g_Unclassified_- } \\
\text { Thiotrichaceaee }\end{array}$ & $\begin{array}{l}\text { S_Unclassified_- } \\
\text { Thiotrichaceae }\end{array}$ & $0.00 \%$ & $0.00 \%$ & $0.00 \%$ & $1.16 \%$ \\
\hline Bacteria & p_Proteobacteria & C_Gammaproteobacteria & o_Thiotrichales & f_Thiotrichaceae & $\begin{array}{l}\text { g_Unclassified_- } \\
\text { Thiotrichaceae }\end{array}$ & $\begin{array}{l}\text { s_Unclassified_- } \\
\text { Thiotrichaceae }\end{array}$ & $0.00 \%$ & $0.00 \%$ & $0.00 \%$ & $0.00 \%$ \\
\hline __Bacteria & p_Proteobacteria & c_Gammaproteobacteria & $\begin{array}{l}\text { O_Unclassified_ } \\
\text { Gammaproteobacteri }\end{array}$ & $\begin{array}{l}\text { f_Unclassified_ } \\
\text { Gammaproteobacteria }\end{array}$ & $\begin{array}{c}\text { g_Unclassified } \\
\text { Gammaproteobacteria }\end{array}$ & $\begin{array}{l}\text { S_Unclassified_- } \\
\text { Gammaproteobacteria }\end{array}$ & $0.03 \%$ & $0.02 \%$ & $0.00 \%$ & $0.06 \%$ \\
\hline Bacteria & p_Proteobacteria & c_Gammaproteobacteria & o_Vibrionales & $\begin{array}{c}\text { f_Pseudo- } \\
\text { alteromonadaceae }\end{array}$ & $\begin{array}{l}\text { g_Unclassified_Pseudo- } \\
\text { alteromonadaceae }\end{array}$ & $\begin{array}{l}\text { s_Unclassified_Pseudo- } \\
\text { alteromonadaceae }\end{array}$ & $0.03 \%$ & $0.00 \%$ & $0.00 \%$ & $0.00 \%$ \\
\hline _Bacteria & p_Proteobacteria & c_Gammaproteobacteria & o_Vibrionales & $\begin{array}{l}\text { fPseudo- } \\
\text { alteromonadaceae }\end{array}$ & g_Vibrio & s_Vibrio_mimicus & $0.00 \%$ & $0.00 \%$ & $0.00 \%$ & $0.00 \%$ \\
\hline Bacteria & p_Proteobacteria & c_Gammaproteobacteria & o_Vibrionales & f_Vibrionaceae & g_Vibrio & s_Unclassified_Vibrio & $0.00 \%$ & $0.00 \%$ & $0.00 \%$ & $0.00 \%$ \\
\hline Bacteria & p_Proteobacteria & c_Gammaproteobacteria & o_Xanthomonadales & f_Sinobacteraceae & $\begin{array}{l}\text { g_Unclassified_ } \\
\text { Sinobacteraceae }\end{array}$ & $\begin{array}{c}\text { S_Unclassified_ } \\
\text { Sinobacteraceae }\end{array}$ & $0.02 \%$ & $0.02 \%$ & $0.00 \%$ & $0.02 \%$ \\
\hline __Bacteria & p_Proteobacteria & c_Gammaproteobacteria & O_Xanthomonadales & f_Xanthomonadaceae & g_Luteimonas & ified_ & $0.00 \%$ & $0.00 \%$ & $0.00 \%$ & $0.01 \%$ \\
\hline Bacteria & p_Proteobacteria & C_Gammaproteobacteria & O_Xanthomonadales & f_Xanthomonadaceae & g_Pseudoxanthomonas & $\begin{array}{l}\text { Pseudoxanthomonas } \\
\text { mexicana }\end{array}$ & $-0.00 \%$ & $0.00 \%$ & $0.00 \%$ & $0.00 \%$ \\
\hline Bacteria & p_Proteobacteria & C_Gammaproteobacteria & O_Xanthomonadales & f_Xanthomonadaceae & g_Stenotrophomonas & $\begin{array}{l}\text { nonas_ } \\
\text { ila }\end{array}$ & $0.00 \%$ & $0.00 \%$ & $0.00 \%$ & $0.00 \%$ \\
\hline Bacteria & p_Proteobacteria & C_Gammaproteobacteria & o_Xanthomonadales & f_Xanthomonadaceae & g_Stenotrophomonas & $\begin{aligned} \text { S_U } \\
\text { Steno }\end{aligned}$ & $0.00 \%$ & $0.00 \%$ & $0.01 \%$ & $0.00 \%$ \\
\hline Bacteria & p_Proteobacteria & c_Gammaproteobacteria & O_Xanthomonadales & f_Xanthomonadaceae & $\begin{array}{c}\text { g_Unclassified_- } \\
\text { Xanthomonadaceae }\end{array}$ & $\begin{array}{r}\text { s_Ur } \\
\text { Xantho }\end{array}$ & $0.02 \%$ & $0.02 \%$ & $0.01 \%$ & $0.01 \%$ \\
\hline __Bacteria & p_Proteobacteria & c_TA18 & O_PHOS-HD29 & $\begin{array}{c}\text { f_Unclassified_PHOS- } \\
\text { HD29 }\end{array}$ & $\begin{array}{c}\text { g_Unclassified_PHOS- } \\
\text { HD29 }\end{array}$ & d_PHOS- & $0.01 \%$ & $0.00 \%$ & $0.00 \%$ & $0.00 \%$ \\
\hline Bacteria & p_Proteobacteria & $\begin{array}{l}\text { C_Unclassified_- } \\
\text { Proteobacteria }\end{array}$ & $\begin{array}{l}\text { O_Unclassified_- } \\
\text { Proteobacteria }\end{array}$ & f_Uncl & $\begin{array}{l}\text { g_Unclassified_- } \\
\text { Proteobacteria }\end{array}$ & $\begin{array}{l}\text { S_Unclas } \\
\text { Proteobe }\end{array}$ & $0.00 \%$ & 0.00 & 0.00 & $0.00 \%$ \\
\hline k_Bacteria & p_SBR1093 & $\begin{array}{l}\text { C_Unclassified } \\
\text { SBR1093 }\end{array}$ & $\begin{array}{l}\mathrm{O}^{-} \mathrm{U} \\
\mathrm{O}\end{array}$ & $\begin{array}{l}\text { fUUnd } \\
\text { SBR }\end{array}$ & $\begin{array}{l}\text { g_Unclassified_ } \\
\text { SBR1093 }\end{array}$ & 93 & $0.04 \%$ & $0.01 \%$ & $0.00 \%$ & $0.00 \%$ \\
\hline Bacteria & p_SBR1093 & c_VHS-B5-50 & $\begin{array}{l}\text { O_Unclassified_- } \\
\text { VHS-B5-50 }\end{array}$ & $\begin{array}{l}\text { fUnc } \\
\text { VHS }\end{array}$ & $\begin{array}{c}\text { g_Unclassified_- } \\
\text { VHS-B5-50 }\end{array}$ & -50 & $0.03 \%$ & $0.01 \%$ & $0.00 \%$ & $0.00 \%$ \\
\hline Bacteria & p_SR1 & C_Unclassified_SR1 & O_Unclassified_SR1 & f_Unclassified_SR1 & g_Uncla & d_SR1 & $0.01 \%$ & $0.00 \%$ & $0.00 \%$ & $0.01 \%$ \\
\hline k_Bacteria & p_Synergistetes & c_Synergistia & o_Synergistales & f_Aminiphilaceae & $\begin{array}{l}\text { g_Unclassified_- } \\
\text { Aminiphilaceae }\end{array}$ & fied_- & $0.00 \%$ & $0.00 \%$ & $0.00 \%$ & $0.01 \%$ \\
\hline Bacteria & p_Synergistetes & c_Synergistia & o_Synergistales & Dethiosulfoyibrionaceae & g_Aminobacterium & $\begin{array}{l}\text { ified_- } \\
\text { erium }\end{array}$ & $0.02 \%$ & $0.01 \%$ & $0.00 \%$ & $0.01 \%$ \\
\hline Bacteria & p_Synergistetes & c_Synergistia & o_Synergistales & & g_TG5 & s_Unclassified_TG5 & $0.02 \%$ & $0.00 \%$ & $0.00 \%$ & $0.00 \%$ \\
\hline __Bacteria & p_Tenericutes & C_CK-1C4-19 & $\begin{array}{c}\text { O_Unclassified_CK- } \\
1 \text { C4-19 }\end{array}$ & _Unclassified_cK- & $\begin{array}{c}\text { g_Unclassified_CK- } \\
1 \mathrm{C} 4-19\end{array}$ & $\begin{array}{r}\text { s_Unclass } \\
1 \mathrm{C} 4 \\
\end{array}$ & $0.09 \%$ & $0.01 \%$ & $0.00 \%$ & $0.00 \%$ \\
\hline Bacteria & p_Tenericutes & c_Mollicutes & o_Acholeplasmatales & f_Acholeplasmataceae & $\begin{array}{c}\text { 9_Unclassified_ } \\
\text { Acholeplasmataceae }\end{array}$ & $\begin{array}{c}\text { S_Unclassified_- } \\
\text { Acholeplasmataceae }\end{array}$ & $0.00 \%$ & $0.00 \%$ & $0.01 \%$ & $0.00 \%$ \\
\hline Bacteria & p_Tenericutes & c_Mollicutes & o_Mycoplasmatales & f_Mycoplasmataceae & $\begin{array}{l}\text { g_Unclassified_- } \\
\text { Mycoplasmataceae }\end{array}$ & $\begin{array}{l}\text { S_Unclassified_- } \\
\text { Mycoplasmataceae }\end{array}$ & $0.00 \%$ & $0.02 \%$ & $0.00 \%$ & $0.00 \%$ \\
\hline Bacteria & p_Tenericutes & c_Mollicutes & o_RF39 & f_Unclassified_RF39 & g_Unclassified_RF39 & RF39 & $0.00 \%$ & $0.00 \%$ & $0.00 \%$ & $0.06 \%$ \\
\hline k_Bacteria & p_Thermotogae & c_Thermotogae & o_Thermotogales & f_Thermotogaceae & g_Thermotoga & ed_- & $0.00 \%$ & $0.00 \%$ & $0.00 \%$ & $0.01 \%$ \\
\hline Bacteria & TM7 & TM7-1 & Unclassified_TM7-1 & f_Unclassified_TM7-1 & g_Unclassified_TM7-1 & ified_TM7-1 & $0.00 \%$ & $0.00 \%$ & $0.00 \%$ & $0.01 \%$ \\
\hline Bacteria & TM7 & TM7-3 & O_CW040 & f_F16 & g_Unclassified_F16 & ied_F16 & $0.00 \%$ & $0.00 \%$ & $0.00 \%$ & $0.01 \%$ \\
\hline Bacteria & TM7 & TM7-3 & o_CW040 & f_Unclassified_CW040 & g_Unclassified_CW040 & d_CW040 & & & 0.0 & $0.00 \%$ \\
\hline Bacteria & p_TM7 & TM7-3 & O_EW055 & f_Unclassified_EW055 & g_Unclassified_EW055 & d_EW055 & $0.01 \%$ & 0.0 & $0.00 \%$ & $0.00 \%$ \\
\hline Bacteria & P_TM7 & C_TM7-3 & O_Unclassified_TM7-3 & 3 f_Uncla & lassified_TM7-3 & TM7-3 & $0.03 \%$ & $0.01 \%$ & $0.00 \%$ & $0.00 \%$ \\
\hline k_Bacteria & $\begin{array}{c}\mathrm{P}_{-} \\
\text {Verrucomicrobia }\end{array}$ & c_[Methylacidiphilae] & o_S-BQ2-57 & $\begin{array}{c}\text { f_Unclassified_S- } \\
\text { BQ2-57 }\end{array}$ & $\begin{array}{c}\text { g_Unclassified_S- } \\
\text { BQ2-57 }\end{array}$ & $\begin{array}{c}\text { S_Unclassified_S- } \\
\text { BQ2-57 }\end{array}$ & $0.01 \%$ & $0.01 \%$ & $0.00 \%$ & $0.00 \%$ \\
\hline __Bacteria & $\begin{array}{c}\mathrm{P}_{-} \\
\text {Verrucomicrobia }\end{array}$ & c_Opitutae & $\begin{array}{c}\text { O_Unclassified_ } \\
\text { Opitutae }\end{array}$ & f_Unclassified_Opitutae & g_Unclassified_Opitutae & s_Unclassified_Opitutae & & & $0.00 \%$ & $0.00 \%$ \\
\hline __Bacteria & $\begin{array}{c}\mathrm{p}_{-} \\
\text {Verrucomicrobia }\end{array}$ & c_Verruco-5 & o_LD1-PB3 & $\begin{array}{l}\text { f_Unclassified_LD1- } \\
\text { PB3 }\end{array}$ & $\begin{array}{c}\text { g_Unclassified_LD1- } \\
\text { PB3 }\end{array}$ & s_Unclassified_LD1-PB3 & $30.01 \%$ & $0.00 \%$ & $0.00 \%$ & $0.00 \%$ \\
\hline Bacteria & $\begin{array}{c}\mathrm{P}_{-} \\
\text {Verrucomicrobia }\end{array}$ & c_Verruco-5 & o_SS1-B-03-39 & $\begin{array}{l}\text { f_Unclassified_- } \\
\text { SS1-B-03-39 }\end{array}$ & $\begin{array}{l}\text { g_Unclassified_- } \\
\text { SS1-B-03-39 }\end{array}$ & & $0.00 \%$ & $0.00 \%$ & $0.00 \%$ & $0.00 \%$ \\
\hline k_Bacteria & $\begin{array}{c}\mathrm{P}_{-} \\
\text {Verrucomicrobia } \\
\mathrm{n}\end{array}$ & c_Verrucomicrobiae & o_Verrucomicrobiales & f_Verrucomicrobiaceae & g_Akkermansia & & $0.03 \%$ & $0.03 \%$ & $0.00 \%$ & $0.08 \%$ \\
\hline K_Bacteria & $\begin{array}{c}\mathrm{P}_{-} \\
\text {Verrucomicrobia }\end{array}$ & c_Verrucomicrobiae & o_Verrucomicrobiales & f_Verrucomicrobiaceae & g_Persicirhabdus & & $0.02 \%$ & $0.00 \%$ & $0.00 \%$ & $0.00 \%$ \\
\hline _Bacteria & $\begin{array}{c}\mathrm{p}_{-} \\
\text {Verrucomicrobia }\end{array}$ & c_Verrucomicrobiae & o_Verrucomicrobiales & f_Verrucomicrobiaceae & $\begin{array}{c}\text { g_Unclassified_ } \\
\text { Verrucomicrobiaceae }\end{array}$ & $\begin{array}{l}\text { s_Unclas } \\
\text { Verrucomic }\end{array}$ & $0.00 \%$ & $0.00 \%$ & $0.00 \%$ & $0.00 \%$ \\
\hline K_Bacteria & $\mathrm{P}_{-}$ & c_Verrucomicrobiae & O_Verrucomicrobiales & f_Verrucomicrobiaceae & g_Verrucomicrobium & $\begin{array}{l}\text { s_Unclas } \\
\text { Verrucomi }\end{array}$ & $0.00 \%$ & $0.00 \%$ & $0.00 \%$ & $0.01 \%$ \\
\hline Bacteria & p_WPS-2 & c_Unclassified_WPS-2 & $\begin{array}{c}\text { O_Unclassified_WPS- } \\
2\end{array}$ & f_Unclassified_WPS-2 & g_Unclassified_WPS-2 & s_Unclassified_WPS-2 & $0.01 \%$ & $0.01 \%$ & $0.00 \%$ & $0.03 \%$ \\
\hline k_Bacteria & p_WS3 & C_PRR-12 & o_Sediment-1 & & g_Unclassified_CV106 & CV106 & $0.00 \%$ & $0.00 \%$ & $0.00 \%$ & $0.00 \%$ \\
\hline k_Bacteria & p_WS3 & C_PRR-12 & o_Sediment-1 & $\begin{array}{l}\text { f_Unclassified_- } \\
\text { Sediment-1 }\end{array}$ & $\begin{array}{l}\text { g_Unclassified__ } \\
\text { Sediment-1 }\end{array}$ & $\begin{array}{l}\text { S_Unclassified__ } \\
\text { Sediment-1 }\end{array}$ & $0.01 \%$ & $0.00 \%$ & $0.00 \%$ & $0.00 \%$ \\
\hline Bacteria & p_WS3 & c_PRR-12 & $\begin{array}{c}\text { o_Unclassified_PRR- } \\
12\end{array}$ & f_Unclassified_PRR-12 & g_Unclassified_PRR-12 & S_Unclassified_PRR-12 & $0.00 \%$ & $0.00 \%$ & $0.00 \%$ & $0.00 \%$ \\
\hline K_Bacteria & P_WS6 & c_SC72 & o_Unclassified_SC72 & f_Unclassified_SC72 & g_Unclassified_SC72 & s_Unclassified_SC72 & 0.0 & & $0.00 \%$ & $0.01 \%$ \\
\hline No blast hit & & & & & & & $0.01 \%$ & 0.00 & 0.6 & $0.01 \%$ \\
\hline
\end{tabular}




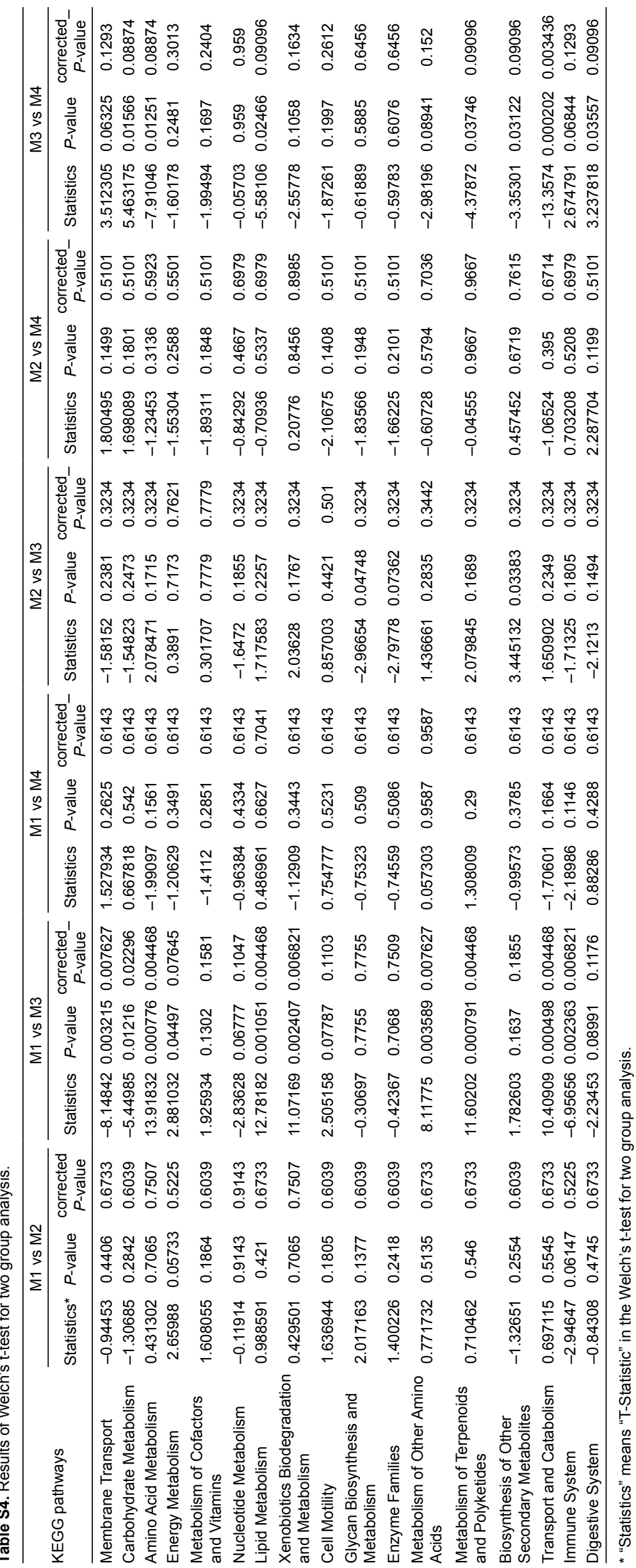

